\title{
A neural circuit for wind-guided olfactory navigation
}

Andrew M.M. Matheson'1, Aaron J. Lanz¹, Angela M. Licata1, Timothy A. Currier ${ }^{1,2, \dagger}$, Mubarak H. Syed ${ }^{3}$, and Katherine I. Nage $1^{1,4^{*}}$

${ }^{1}$ Neuroscience Institute, NYU Medical Center, 435 E 30th St. New York, NY 10016, USA

${ }^{2}$ Center for Neural Science, NYU, New York, NY, 4 Washington Place, New York, NY 10003

${ }^{3}$ Department of Biology, 219 Yale Blvd NE, University of New Mexico, Albuquerque, NM 87131

† Present address: Dept. of Neurobiology, Stanford University, 299 W. Campus Drive, Stanford CA 94305

${ }^{*}$ Corresponding author. Email: katherine.nagel@nyumc.org

\section{Abstract:}

To navigate towards odor in a turbulent environment, animals integrate odor value cues with wind direction cues. The central neural circuits subserving this behavior are unknown. Here we used optogenetic activation to identify neurons in the lateral horn (LH), mushroom body (MB), and fanshaped body (FB), that drive upwind orientation in walking Drosophila. Using calcium imaging, we show that MB/LH neurons encode odor independent of wind direction, and that odor and wind are integrated within $\mathrm{h} \Delta \mathrm{C}$ local neurons of the $\mathrm{FB}$ to drive re-orientation. Based on connectome data, we model an FB circuit that allows odor to switch behavioral orientation to wind. Our work identifies central neural circuits that integrate value and direction signals to generate an essential goal-directed behavior. 


\section{Introduction}

Navigation frequently involves the integration of value cues that indicate the identity of important resources, with spatial and directional cues that allow for motor planning (1,2). A natural example of this occurs in wind-guided olfactory navigation $(3,4)$, in which the scent of food, or a potential mate, gates orientation relative to wind direction. This basic algorithm has been observed in diverse species (5-7), and allows animals to solve the problem of locating an odor source in turbulence $(8,9)$. In insects, numerous theoretical studies have proposed that the output of the mushroom body, a brain structure involved in olfactory and visual memory, might encode stimulus value (10-12), whereas the integration of value and direction cues might occur within the central complex (13-16). However, experimental data and precise functional circuits supporting these hypotheses have been lacking.

Here, we employ an optogenetic navigation paradigm in walking Drosophila (8), 2-photon calcium imaging, and analysis of the hemibrain connectome (16-19) to identify neural circuits that integrate odor and wind cues to drive goal-directed navigation behavior. After establishing our paradigm using peripheral olfactory neurons, we show that specific output neurons of two high-order olfactory areas - the lateral horn and mushroom body - encode a non-directional odor signal capable of driving robust upwind orientation. We next identify several downstream fan-shaped body inputs that likewise drive upwind orientation and encode odor. In a previous study, we identified a set of fan-shaped body columnar neurons (PFNa, $\mathrm{p}$ and $\mathrm{m}$; here called windPFNs) that encode wind direction independent of odor (20). These PFNs are distinct from those recently shown to encode self-motion information (PFNd and PFNv, 21, 22). By searching for neurons that receive input from both windPFNs and olfactory FB inputs, we identify a group of local fan-shaped body neurons, $\mathrm{h} \Delta \mathrm{C}$ neurons, that integrate odor and wind direction signals and drive turning behavior when activated. Finally, we develop a model based on EM connectivity that shows how this circuit can produce flexible orientation to wind determined by odor context. These experiments delineate a specific neural circuit that integrates olfactory value cues with mechanosensory direction cues to generate a goal-directed orientation behavior. This basic circuit architecture may be used with other modalities to generate flexible goal-oriented navigation in complex environments.

\section{RESULTS}

\section{An optogenetic paradigm to investigate the neural circuit basis of upwind orientation}

To identify neural circuits that drive olfactory navigation behaviors, we developed an optogenetic activation paradigm in a set of miniature wind tunnels (Fig. 1A, modified from 8). We first asked whether optogenetic activation of olfactory receptor neurons (ORNs) with Chrimson could produce behavioral phenotypes similar to those observed with an attractive odor. We found that broad activation of olfactory receptor neurons using either the orco, or orco and IR8a co-receptor promoters together, $(23,24)$ resulted in robust navigation behaviors similar to those observed with apple cider vinegar (ACV, Fig. 1B,C, Fig. S1A). In response to either odor or light, flies ran upwind, generating an increase in 
upwind velocity. Following odor or light offset, they initiated a local search, characterized by increased curvature (Fig. 1B,C). Neither behavior was observed in the absence of the orco or orco/IR8a-GAL4 driver, or when we expressed Chrimson under an empty-GAL4 or empty split-GAL4 driver (Fig. 1D, Fig. S1B). Silencing both orco and IR8a-positive ORNs using tetanus toxin abolished both upwind and search responses to odor (Fig. 1E). Thus, optogenetic activation can substitute for odor in producing upwind orientation and offset search, and ORNs are required for these behavioral responses to odor.

ACV activates a subset of both orco+ and IR8a+ glomeruli (25). Although the behavioral phenotypes evoked by ACV and by optogenetic activation of ORNs were similar, they exhibited some subtle differences. The offset search behavior evoked by optogenetic activation of either orco/IR8aGAL4 or orco-GAL4 alone was more robust than that evoked by ACV (Fig. 1C,D, Fig. S1A). In contrast, in the same flies, ACV produced a stronger upwind response than optogenetic activation of orco+ ORNs (Fig. S1A). Activation of orco/IR8a+ ORNs in the absence of wind produced offset search without upwind orientation (Fig. S1C) indicating that both olfactory neuron activation and wind together are required for upwind orientation. Activation of single ORN types known to be activated by ACV (25), did not generate significant upwind orientation or offset search and silencing of orco+ or IR8a+ ORNs alone did not abolish upwind orientation (Fig. 1F, Fig. S1E). Overall, these results suggest that upwind orientation and offset search can be evoked independently and are driven by distinct but overlapping populations of olfactory glomeruli. They also indicate that substantial silencing of most olfactory neurons is required to abolish upwind orientation to ACV.

\section{A subset of LHONs and MBONs drive wind orientation and encode a non-directional odor signal}

We next turned our attention to output neurons of two higher-order olfactory areas, the lateral horn (LH) and mushroom body (MB) $(10,18,19,26)$. We activated several groups of output neurons (henceforth: LHONs and MBONs) that were previously shown to produce attraction in quadrant assays, and have been proposed to encode odor valence $(10,26)$. We found that several of these drove upwind orientation when activated (Fig, 2A,B, Fig. S2A, 10). Neurons promoting upwind orientation included the cholinergic LHON cluster AD1b2 (labeled by LH1396, LH1538, and LH1539 (19, 26, 27), Fig. 2A), and the cholinergic MBON lines MB052B (labeling MBONs 15-19), MB077B (labeling MBON12), and MB082C (labeling MBONs 13 and 14, Fig. 2B). AD1b2 drivers and MB052B also elicited significant increases in offset curvature when activated (Fig. 2A,B). Activating individual MBONs within MB052B (MBONs15-19) did not drive significant upwind orientation (Fig S2B). In contrast, the glutamatergic MBON line MB434B (labeling MBONs 5 and 6), which was previously shown to produce aversion (10), generated downwind orientation in our paradigm (Fig. 2C). Moreover, two MBON lines produced straightening (reduced curvature) in our paradigm (Fig. 2D) but no change in wind orientation (Fig. 2C, Supp. Fig. 2B): the GABAergic line MB112C (labeling MBON 11), which evoked attraction in quadrant assays (10), and the glutamatergic line MB011B (labeling MBONs 1,3,4), which evoked aversion. Silencing single MBON or LHON lines that drove navigation phenotypes did not abolish upwind orientation in response to odor (Fig. S2C). Overall these results indicate that LH/MB outputs can drive coordinated "suites" of locomotor behavior that promote attraction or aversion in different environments. Several LHONs and MBONs redundantly drive upwind orientation, a key behavior for attraction in windy 
environments, while other MBONs drive straightening, which promotes attraction in odor gradients (28) or in response to familiar visual stimuli (12). Subsets of attractive and aversive MBONs drive upwind and downwind orientation, indicating that the MB can promote opposing behaviors. Broadly, these results suggest that the MB and LH can transform sensory representations into specific locomotor programs rather than simply encoding stimulus valence.

Are the LHONs and MBONs that drive upwind orientation themselves sensitive to wind direction, or do they encode an odor signal that is integrated with wind direction information downstream? To answer this we performed calcium imaging experiments from the two lines that generated the strongest upwind orientation - LH1396 and MB052B - while presenting the same calibrated odor stimulus from 5 different directions (29, Fig. S2D). Across flies we found that both lines responded robustly to $A C V$, but did not show consistent modulation by wind-direction (Fig. 2E, Fig. $\mathrm{S} 2 \mathrm{E})$. Previous studies found that the a'3 compartment of the MB is responsive to airflow (30), and that a'3 MBONs receive putative mechanosensory input from the WED (27). To test whether these neurons show wind direction tuning, we performed whole-cell recordings from these neurons using the splitGAL4 line MB027B, while presenting odor from one side. We patched both ipsilateral and contralateral a'3 MBONs and found no significant difference in their response to ACV (Fig. 2F). Together these results suggest that odor and wind direction signals are integrated downstream of the MB and $\mathrm{LH}$.

\section{Multiple inputs to the fan-shaped body respond to odor and promote upwind orientation}

Where might olfactory signals from the $\mathrm{MB}$ and $\mathrm{LH}$ be integrated with wind direction signals to guide orientation? Two recent studies identified the fan-shaped body (FB), which receives wind direction information through windPFNs (20), as a major downstream target of the $\mathrm{MB}(18,31)$. We performed anterograde trans-synaptic tracing (32) on two of our lines (MB052B and LH1396) that drove upwind orientation and observed signal in the dorsal layers of the fan-shaped body in both cases (Fig $3 \mathrm{~A}$ ). We therefore sought downstream targets of the $\mathrm{MB}$ and $\mathrm{LH}$ that might drive wind orientation phenotypes in the FB.

We took two approaches to identify FB neurons involved in wind-guided olfactory navigation. First, we performed an optogenetic activation screen of 77 driver lines labelling central complex (Cx) neurons, (35 Janelia Flylight, 29 Vienna Tiles collection, and 13 split GAL4 lines generated in this study, 33, 34, 35), of which 40 labeled FB input neurons (Fig. 3B and Fig. S3). We performed this screen using genetically blind flies (see Materials and Methods) and in the presence of teashirt-Gal80 (36) to reduce potential Chrimson expression in the ventral-nerve cord (VNC) (see Material and Methods). A small number of lines drove significant increases in upwind velocity, while most did not, although many showed an increase overall movement (Fig. 3B). We subsequently imaged lines that showed significant increases in upwind velocity to see if they responded to ACV. Second, we used the hemibrain connectome (17) to identify FB inputs downstream of the MBONs and LHONs that showed wind orientation phenotypes (Fig. S4A). We then identified lines that label these FB inputs, and tested them for ACV responses and behavioral activation phenotypes. Through these two approaches we 
identified at least 3 distinct groups of FB inputs that respond to ACV and drive upwind orientation when activated.

We identified the neuron FB5AB using the connectome (Fig. 3C, Fig. S4A,B). This neuron stood out as the only FB input that receives at least one direct synaptic input from each of the MBON lines with wind orientation phenotypes (Fig. S4A). In addition, FB5AB receives indirect input from AD1b2 LHONs through the SIP interneurons, LCENT3 and LHPV5e1 (Fig. S4A). Finally FB5AB receives the largest number of disynaptic LH inputs from ACV-responsive glomeruli of any FB input neuron (Fig. S4B, Methods). We identified a GAL4 line that labels FB5AB neurons (21D07-GAL4), and used this to image calcium responses to ACV. FB processes in this line showed robust responses to ACV delivered from all directions, although the most consistent responses were from the front (Fig. 3C, Fig. S4C). As 21D07-GAL4 labels some neurons in the antennal lobe, a primary olfactory area, we used the CLIN technique (37) to limit expression of Chrimson to neurons in the $\mathrm{Cx}$ (see Methods). This technique weakly but specifically labeled FB5AB (Fig. 3C). Light activation of this driver at high intensity drove upwind orientation (Fig. 3C). We found that FB5AB is cholinergic (excitatory, Fig. S4E), and receives excitatory cholinergic input from the attractive MBONs 12, 13, and 19 and inhibitory glutamatergic input from the aversive MBON5 (Fig. S4A). Thus FB5AB is a downstream target of both the $\mathrm{MB}$ and $\mathrm{LH}$ that encodes odor and drives upwind orientation.

We identified a second group of olfactory upwind-promoting neurons through our screen. The line 65C03-GAL4 labels several neurons that receive input in SIP/SMP and project to the dorsal FB, with some inputs to the ventral FB as well (Fig. 3D). Activation of 65C03-GAL4 neurons drove robust upwind orientation without local search at offset (Fig. 3D). Imaging from the dorsal FB processes of 65C03-Gal4 showed small responses to ACV without strong directional tuning (Fig. 3D). Two other dorsal FB lines drove upwind orientation: 45D04-GAL4 and 12D12-GAL4, but we did not observe calcium response to odor in 45D04-GAL4. We attempted to isolate upwind-promoting neurons in these lines by building split-GAL4 lines with these hemi-drivers (35) but none of these produce upwind orientation (Fig. 3B). Two sleep-promoting dorsal FB input lines (23E10-GAL4 and 84C10-GAL4, 38) did not produce upwind orientation or offset search in our paradigm (Fig. 3B) indicating that dorsal inputs to the FB play diverse roles in behavior.

Finally we identified a set of ventral FB inputs that drove upwind orientation (Fig. 3E). We initially identified these neurons labeled by the line VT029515-GAL4 (Supp. Fig. 4G). We also generated a split-GAL4 line (vFB split: 13B10AD;VT041421DB) that specifically labels inputs from the Antler (ANT) to the ventral FB and drives weak upwind orientation (Fig 3E). Imaging from vFB split neurons showed robust non-directional responses to wind onset with smaller and more variable responses to odor (Fig. 3E, Fig. S4C). We previously recorded strong wind responses in VT029515GAL4 without direction tuning (20). In separate experiments we found that a pulse of un-odorized wind drives a transient upwind orientation (followed by downwind orientation) that contrasts with the persistent upwind orientation driven by odor (Fig. S4H). Given that wind information is strongly represented in the ANT (29), we hypothesize that these vFB neurons may be involved in the transient upwind orientation produced by wind. 
Together, these data indicate that multiple inputs to the FB carry odor information and drive upwind orientation. Silencing of single FB inputs did not abolish the upwind response to odor (Fig. S4I) suggesting that they work redundantly to promote upwind orientation. Olfactory FB inputs showed more frontal tuning than $\mathrm{LH} / \mathrm{MB}$ outputs, but no tuning to wind directions away from the front. In a previous study, we identified windPFNs as FB inputs that are strongly tuned to wind at $+/-45^{\circ}$ and not strongly modulated by odor (20). Thus, we hypothesized that odor and wind direction information might be integrated within local neurons of the FB.

\section{$\mathrm{h} \Delta \mathrm{C}$ neurons integrate odor and wind direction signals and drive re-orientation}

To identify FB local neurons that might integrate odor and wind direction signals, we used the connectome to search for neurons that receive input both from FB5AB and from windPFNs. This analysis revealed a population known as $h \Delta C$ neurons that tile the vertical columnar structure of the FB (Fig. 4A, 16). Each $\mathrm{h} \triangle \mathrm{C}$ neuron receives input from both left and right windPFNs at their ventral dendrites, and from FB5AB at their dorsal axons, where they could gate synaptic output (Fig. 4B, C).

To ask whether $\mathrm{h} \triangle \mathrm{C}$ neurons integrate wind and odor signals, we identified a GAL4 lineVT062617 - that labels $\mathrm{h} \Delta \mathrm{C}$ neurons and performed calcium imaging from the FB while presenting odor and wind from different directions. We observed calcium responses mostly in the dorsal fan, where $\mathrm{h} \Delta \mathrm{C}$ neurons form output tufts (Fig. 4D), thus we expect that these responses arise largely from output processes (22). In several examples, we observed odor responses that were strongest for a particular direction of wind (Fig 4E). Across recordings, responses were strongest to +45 and $-45^{\circ}$ (Fig. $4 \mathrm{~F}$ ), similar to windPFNs, although the relationship between wind direction and peak response was not consistent across flies (Fig. 4E). VT062617-GAL4 neurons responded more strongly to odor than to wind onset (Fig. 4G, Supp. Fig. 4D) and odor responses were typically localized to a few nearby columns (Fig. 4H). We found VT062617-GAL4 neurons to be cholinergic (Fig. S4E) and thus excitatory. Broadly these results are consistent with the idea that $\mathrm{h} \Delta \mathrm{C}$ neurons receive and integrate both odor information and wind-direction information. In particular, they suggest that wind-direction tuned activity in $\mathrm{h} \Delta \mathrm{C}$ neurons is gated on by odor.

To determine whether $\mathrm{h} \Delta \mathrm{C}$ neurons contribute to navigation, we generated two split-GAL4 lines that selectively label $\mathrm{h} \Delta \mathrm{C}$ neurons. Activation of these lines caused flies to turn, resulting in an increase in curvature (Fig 4H, Fig. S4J). No such increase in curvature was observed with empty-GAL4 or empty split-GAL4 drivers (Fig. 3B). We also observed a strong turning phenotype when activating VT062617GAL4 (Fig. S4F). Our results thus support the idea that $\mathrm{h} \Delta \mathrm{C}$ neurons integrate wind direction and odor inputs to drive re-orientation behavior.

\section{The architecture of the FB suggests a model for olfactory gating of wind orientation}

Our data suggest that olfactory inputs to the FB - terminating at $\mathrm{h} \Delta \mathrm{C}$ neurons - are capable of rapidly switching behavioral orientation to wind from a baseline downwind orientation (8) to upwind 
orientation. How might FB circuits enable flies to flexibly orient to wind depending on odor context? The FB receives wind direction input through windPFNs (20) and has been proposed to control steering motor output through PFL3 output neurons $(16,39)$. We therefore hypothesized that competing pathways between these two groups of neurons might promote down- and up-wind orientation respectively, with odor input through $\mathrm{FB} 5 \mathrm{AB}$ and other input neurons altering the balance of activity between these pathways. WindPFNs make both direct connections onto PFL3 neurons (Fig. 5A) and indirect connections onto PFL3 neurons via $\mathrm{h} \triangle \mathrm{C}$ and FC neurons (Fig. 5B, 16). Thus, the connectivity of the FB supports the notion of competing pathways from wind direction input to pre-motor output, with the indirect pathway gated by odor input.

To explore whether these two pathways could account for behavioral orientation to wind, we next examined the relationship between wind direction and motor output in our behavioral data (8). We plotted the average angular velocity as a function of wind orientation both at baseline (Fig. $5 \mathrm{C}$ ) and in the presence of odor (Fig. 5D). In the wind only condition (Fig. 5C), wind from the left (negative wind direction) promotes right turns (positive turn angle), leading to stable downwind orientation. In contrast, in the wind + odor condition (Fig. 5D), wind from the left promotes turns to the left, and vice-versa, leading to stable upwind orientation. Thus, up- and down-wind orientation reflect distinct mappings from wind input to steering motor output.

Can circuit connections between windPFNs and PFL3 neurons account for these mappings? To address this question, we developed a computational model of neural activity in windPFNs and PFL3 neurons (Fig. 5E-J). Each of these populations consists of two sets of columnar neurons that innervate the left or right protocerebral bridge respectively, then converge in the FB (Fig. 5E,H). Recent studies have suggested that these populations can represent vector information as sinusoidal bumps of calcium activity across the columns of the FB $(16,21,22,40)$. The vector angle is given by the location of the bump along the FB and the vector magnitude is given by the amplitude of the bump. Vector angles are inherited from the compass system in the ellipsoid body and have specific anatomic offsets relative to this global heading signal (16). Vector magnitudes depend on other inputs to each population. For example, windPFNs receive wind direction input in the noduli (20), while PFL3 neurons receive input from PFNs and indirectly from $h \triangle C$ (16).

In our model, left and right windPFNs have vector angles that are shifted by $45^{\circ}$ contralateral to the heading compass, following anatomical data (16, Fig. 5E). Vector magnitudes in these neurons depend sinusoidally on wind direction, and are maximal at $+45^{\circ}$ for left PFNs and $-45^{\circ}$ for right PFNs, following physiological data (20, Fig. $5 \mathrm{~F})$. Activity profiles in left and right windPFNs sum in the FB to produce an overall wind vector that is closer to the left windPFN bump when wind comes from the left and vice-versa (Fig. 5G). In contrast, activity in $\mathrm{h} \Delta \mathrm{C}$ neurons is projected half-way across the $\mathrm{FB}$, resulting in a wind vector that is rotated by $180^{\circ}$ (Fig. $5 \mathrm{G}$ ). Thus, the direct and indirect pathways produce two competing wind vectors in the FB that differ by $180^{\circ}$ much like behavioral orientation down- and upwind. 
PFL3 neurons then translate these wind vectors into steering commands $(39,40)$. Left and right PFL3 neurons have heading vector angles that are each shifted by $90^{\circ}$ ipsilateral to the compass, producing two sinusoidal profiles that are separated by $180^{\circ}(16$, Fig. $5 \mathrm{H})$. To compute activity in right and left PFL3 neurons, we project (sum) the sinusoids representing wind vectors in either windPFNs or $\mathrm{h} \Delta \mathrm{C}$ with these right and left PFL3 heading sinusoids (Fig. $5 \mathrm{I}, \mathrm{J}$ ). We then compute turn direction by calculating the ratio of right to left PFL3 activity. In essence, this circuit compares each wind vector to the two PFL3 vectors and votes for whichever one is more similar to the wind vector.

In the direct pathway (Fig. 5I), wind on the left produces a windPFN vector that overlaps more closely with the right PFL3 sinusoid, producing a right turn. PFL3 neurons project contralaterally, and we assume they are inhibitory, generating an ipsilateral turn. When simulated for all possible wind directions, this model generates a downwind mapping between wind direction and motor output, similar in shape to the one measured experimentally (Fig. 5K, Supp Fig. 5). In the indirect pathway (Fig. 5J), wind on the left produces a wind vector in $\mathrm{h} \Delta \mathrm{C}$ that overlaps more closely with the left PFL3 sinusoid, producing a left turn. Across wind directions, this circuit generates an upwind mapping from wind direction to motor output (Fig. 5L, Fig S5). A similar set of computations suggests that h $\Delta \mathrm{C}$ might also alter forward walking speed as a function of wind direction, mediated by PFL2 output neurons (Fig. S6). Overall, the circuit logic outlined here allows a non-directional value signal (in this case carried by FB5AB) to alter the relationship between directional sensory input (carried by windPFNs) and motor output (carried by PFL3 and PFL2), resulting in a change to the animal's target heading.

\section{Discussion}

\section{Neural circuits for value and direction integration}

Navigation frequently requires both the representation of value information, for example the identities and estimated values of different food sources or conspecifics, and of spatial and directional information, such as one's location in a room. Both of these information sources must be combined to effectively plan navigational and locomotor programs. In vertebrates, value information is most prominently represented in the striatum, VTA, and substantial nigra (41-43), while spatial information is represented in the hippocampus and entorhinal cortex (44-45) although recent data suggest that many brain structures multiplex both types of information (1,2). A key question in neuroscience is thus what type of circuit architectures might allow for effective and flexible integration of these two types of information.

In insects, previous research has proposed the mushroom body computes valence or value information, both for olfactory cues (46), and for visual cues (47), while the lateral horn clusters olfactory input according to its innate valence $(19,26)$. In contrast, the central complex represents variables such as heading direction (48) and self-motion in both ego-and allo-centric coordinates $(21,22)$ and plays a key role in spatial navigation $(13,15,16)$. Several recent theoretical studies have proposed ways that the mushroom body and central complex might work together to promote navigation towards a goal (12-16), particularly for visually-guided navigation. Specifically, they propose 
that tangential inputs to the FB, many of which are anatomically downstream of the $\mathrm{MB}(18,29)$, carry contextual information that allows a fly to select different navigational programs (12-16). However, experimental support for these models has been lacking.

Wind-guided olfactory navigation presents an ethological model for the integration of value information and spatial or directional information. Attractive food and mate odors drive robust orientation upwind (3-9). Attraction and repulsion can be both innate or learned $(10,11,25,46)$. Thus, the odor attractiveness is thought to reflect the output both of hard-wired pathways in the lateral horn $(26,19)$, and of mushroom body circuits that update odor valence based on its association with rewards and punishments $(10,11,46,49)$. Wind direction is also an abstract quantity that must be inferred from a variety of primary sensory inputs. In walking flies, wind direction is computed by comparing mechanosensory displacements of the two antennae $(50-52,29)$. In flight, however, wind displaces the fly as a whole, and wind direction is thought to be inferred from a comparison of expected and actual optic flow (53-54). Recent studies have identified inputs to both the ellipsoid body and fan-shaped body that encode wind direction based on mechanosensory cues $(52,20)$, while other fan-shaped body neurons have been shown to encode optic flow relative to intended heading $(21,22)$. We therefore hypothesize that the FB may compute a representation of wind direction independent of whether the fly is walking or flying. Separating the computation of stimulus value (in the MB/LH) from the computation of wind direction (in the FB) may allow flies to generalize stimulus associations learned in one context (walking) to another (flight).

Here we provide experimental evidence that directionless value cues represented in the outputs of the MB and $\mathrm{LH}$ are transformed into directional navigation cues within the fan-shaped body of the central complex. We showed that outputs of both the $\mathrm{MB}$ and $\mathrm{LH}$ are sufficient to drive upwind orientation when activated but encode attractive odor in a non-directional manner. We identified and characterized olfactory inputs to the fan-shaped body, showing that these likewise encode odor identify and drive upwind orientation. We observed weak frontal tuning in some of these inputs, perhaps reflecting feedback from FB local neurons. However, none showed tuning to directions away from the midline. In contrast, $\mathrm{h} \Delta \mathrm{C}$ local neurons exhibited odor responses that were strongest at directions of $+/-45^{\circ}$, similar to their windPFN inputs. Finally we showed that $\mathrm{h} \Delta \mathrm{C}$ neurons can drive changes in orientation. Together our results identify a functional circuit that links the MB/LH to the central complex to promote a specific goal-directed navigation behavior (Fig. 6).

\section{A model for encoding of goal orientations in the fan-shaped body}

How is the Cx able to encode and alter the animal's goal orientation? Here we propose a model, based on functional and connectomic data, of how odor input through FB5AB could switch orientation to wind direction cues. In this model, competing direct and indirect pathways link wind direction cues, carried by windPFNs, to pre-motor outputs, carried by PFL3 neurons. h $\Delta \mathrm{C}$ interneurons are able to invert the mapping from windPFNs to PFL3s through their horizontal projections that rotate their wind vector representation by $180^{\circ}$. FB5AB is able to gate this indirect pathway ON through its excitatory synapses onto the output tufts of $h \Delta C$ neurons. This model differs from one recently 
proposed for $\mathrm{h} \triangle \mathrm{B}$ neurons, where the $180^{\circ}$ offset between input and output served to pool sensory input from two types of PFNs that signal the same allocentric traveling direction $(21,22)$. Unlike $h \Delta B$, $\mathrm{h} \triangle \mathrm{C}$ neurons receive PFN input only on their dendrites. Thus, these two cell types with similar morphology may implement distinct computations through their different arrangement of anatomical inputs.

In our model, the representation of wind direction in $\mathrm{h} \Delta \mathrm{C}$ neurons is not fixed, but depends on the fly's heading compass (Fig. S5). This is because $\mathrm{h} \Delta \mathrm{C}$ receives input from windPFNs, which receive a fixed ego-centric wind direction input through the noduli (20) and a fly-specific heading signal through the proto-cerebral bridge (16). h $\Delta \mathrm{C}$ output is further gated by odor information carried by FB5AB. Our model is broadly consistent with the observation that $\mathrm{h} \triangle \mathrm{C}$ neurons responded most prominently during the odor phase, and that directional tuning for wind differs across flies. However, a full test of this model will require simultaneous imaging of compass signals and $\mathrm{h} \Delta \mathrm{C}$ signals, likely in behaving flies. An intriguing feature of our model is that - although the representation of wind direction in $\mathrm{h} \Delta \mathrm{C}$ depends on allocentric heading - the behavioral output of the model depends only on the ego-centric angle of the wind with respect to the fly (Fig. S5). This representation in $\mathrm{h} \Delta \mathrm{C}$ might facilitate the integration of wind direction cues with allocentric visual or memory-guided cues for navigation, as has been observed in ant navigation where path integration and plume tracking can compete during foraging and homing (55).

Our current model provides a minimal account of circuits consistent with our data that could convert a directionless odor signal into a switch from downwind to upwind orientation. However, the actual function of this circuit in vivo is likely to be more complex. First, we showed that several other FB neurons besides FB5AB encode odor signals and can evoke upwind orientation (Fig. 3). Further, our screen revealed additional FB neuron types that drove changes in wind orientation (Fig. S3). A handful of other local neuron types in the FB also receive co-incident input from both FB5AB and windPFNs. We hypothesize that such circuits could help stabilize the upwind versus downwind arms of the circuit during odor, or allow the circuit to specify orientation at angles other than directly up- or downwind. Second, the FB contains other output pathways besides PFLs, and these might also contribute to motor output. Finally, we were not able to abolish upwind orientation by silencing any of the individual neurons in our pathway (Fig. S4). This suggests that there might be additional pathways bypassing the FB that contribute to wind orientation. For example, direct pathways linking the $\mathrm{MB}$ to the LAL have recently been described (31) and these might allow for a more direct influence of odor signals on ego-centric wind representations. Future studies should elucidate the full complement of neurons involved in wind-guided olfactory navigation.

The ability to navigate towards sources of attractive odor is fundamental for both foraging and reproduction in most species. At the macroscopic scales we are most familiar with, odors form turbulent plumes, in which chemical information alone is insufficient to locate an odor source $(3,4)$. Numerous behavioral studies, in organisms from insects, crustaceans and mammals, have demonstrated that flow information, derived from either mechanosensation or optic flow, must be combined with odor information to solve this task $(5-9,54)$. Here we have identified central neural 
circuits in conserved regions of the arthropod brain that integrate odor and airflow signals to enable goal-directed navigation (Fig. 6). The identification of such circuits will enable a quantitative understanding of how these fundamental search behaviors are encoded and have diversified across evolution.

\section{Acknowledgements}

The authors would like to thank Marc Gershow, Karla Kaun, Michael Dickinson, Michael Reiser, Matthew Clark, Matthieu Louis, Richard Mann, Gilad Barnea, Rachel Wilson, Tzumin Lee, Janelia Flylight, and the Bloomington and Vienna Drosophila Resource Centers for fly stocks. We thank Michael Long, Elizabeth Hong, Floris van Breugel, and members of the Nagel and Schoppik labs for helpful input on the manuscript. This work was supported by R01DC017979, NSF Ideaslab and NSF Neuronex grants, as well as a McKnight Scholar Award to K.I.N. T.A.C was supported by a Dean's Dissertation Fellowship from NYU.

\section{Author contributions:}

K.I.N and A.M.M.M conceived the project, with a major conceptual contribution by A.J.L. A.M.M.M performed the vast majority of behavioral, imaging, electrophysiology, and anatomy experiments, and generated new genetic stocks. A.J.L. performed connectomic analysis and developed the computational model with input from K.I.N. and A.M.M.M. A.M.L. and A.J.L contributed to behavioral experiments. T.A.C. performed a subset of electrophysiology experiments. M.H.S. provided the genetic strategy to isolate Cx neurons. K.I.N, A.M.M.M, and A.J.L wrote the paper with input from the other authors.

Competing Interests: The authors declare no competing interests.

Data and materials availability: All data will be made available on Dryad on publication. Software will be made available on Github on publication.

\section{Materials and Methods}

FLY STRAINS

All experimental flies (except flies for trans-tango described below) were raised at $25^{\circ} \mathrm{C}$ on a standard cornmeal-agar medium. Flies were raised with a $12 \mathrm{~h}$ light-dark cycle. We used the following genotypes and ages for the experiments shown in each figure. Parental genotypes and sources are listed in the key resources table. For optogenetic activation experiments, experiments were run in male norpA hemizygotes to eliminate any possible innate visual responses to red light. All other flies used were female. We detected no difference in olfactory behavior of male versus female flies in our assay in a previous study (8). For calcium imaging experiments we used older flies (5-21 days) to maximize indicator expression. For electrophysiology we used younger flies to minimize glial ensheathing which 
make it challenging to obtain clean patch recordings. Trans-tango flies were raised at $18^{\circ} \mathrm{C}$ and aged until they were 10-20 days old following recommended protocols (32).

\begin{tabular}{|c|c|c|c|}
\hline Genotype & Description & $\begin{array}{l}\text { Age } \\
\text { range }\end{array}$ & Figure panels \\
\hline \multicolumn{4}{|l|}{ Figure 1} \\
\hline $\begin{array}{l}\text { norpA }{ }^{36 / y} \text {;UAS-Chrimson- } \\
\text { mVenus/orco-GAL4;UAS-10xGFP/+ }\end{array}$ & $\begin{array}{l}\text { genetically blind male flies } \\
\text { expressing Chrimson under the } \\
\text { orco promoter }\end{array}$ & $3-10$ days & $\begin{array}{l}\text { ACV experiments in } \\
\text { Fig. 1B,C,D, Supp. } \\
\text { Fig 1A, Fig. 5A, } \\
\text { Supp. Fig. 6A } \\
\text { orco activation in } \\
\text { Fig. 1D, Supp. Fig. } \\
\text { 1A }\end{array}$ \\
\hline $\begin{array}{l}\text { norpA }{ }^{36 / y ; t s h-g a l 80 / o r c o-G A L 4, I R 8 a-~} \\
\text { GAL4;UAS-Chrimson-mVenus/+ }\end{array}$ & $\begin{array}{l}\text { genetically blind male flies } \\
\text { expressing Chrimson under the } \\
\text { orco and IR8a promoters }\end{array}$ & 3-10 days & $\begin{array}{l}\text { Optogenetic } \\
\text { activation } \\
\text { experiments in Fig. } \\
\text { 1B,C,D, Fig. S1C }\end{array}$ \\
\hline $\begin{array}{l}\text { norpAss/y;UAS-Chrimson- } \\
\text { mVenus;UAS-10xGFP x X-GAL4 or } \\
\text { split-GAL4 } \\
\text { X-GAL4: orco-GAL4,IR8a-GAL4 (II), } \\
\text { empty-GAL4 (II), empty split-GAL4 } \\
\text { (II,III), OR59a (III), OR42a (III), IR75a } \\
\text { (III), IR64a (II), OR92A (III), OR42b (II) }\end{array}$ & $\begin{array}{l}\text { genetically blind hemizygous } \\
\text { male flies expressing Chrimson } \\
\text { under various GAL4 or split-GAL4 } \\
\text { drivers on chromosomes II or III. }\end{array}$ & 3-10 days & $\begin{array}{l}\text { optogenetic } \\
\text { activation } \\
\text { experiments in Fig. } \\
\text { 1B,C,D, Fig. S1B,D }\end{array}$ \\
\hline $\begin{array}{l}\text { norpA } 36 / y ;+/ U A S-C h r i m s o n- \\
\text { mVenus;+/UAS-10xGFP }\end{array}$ & parental control & 3-10 days & $\begin{array}{l}\text { parental control for } \\
\text { activation in Fig. 1D }\end{array}$ \\
\hline $\mathrm{UAS}^{\mathrm{T}} \mathrm{TNT}_{5005}$ & $\begin{array}{l}\text { parental control; UAS-TNT } \\
\text { backcrossed } 5 \text { generations to } \\
\text { w1118 } 5905\end{array}$ & 3-10 days & $\begin{array}{l}\text { parental control for } \\
\text { silencing in Fig. 1E,F }\end{array}$ \\
\hline $\begin{array}{l}\text { UAS-TNT }_{5005} \times \text { X-GAL4 } \\
\text { X-GAL4: orco-GAL4, IR8a-GAL4, } \\
\text { orco-GAL4,IR8a-GAL4 }\end{array}$ & flies with various ORNs silenced & 3-10 days & Fig. 1E, F, Fig. S1E \\
\hline \multicolumn{4}{|l|}{ Figure 2} \\
\hline $\begin{array}{l}\text { norpA36/y;tsh-Gal80;UAS-Chrimson- } \\
\text { mVenus x X-GAL4 or split-GAL4 } \\
\text { X-GAL4/split-GAL4: LH1538, } \\
\text { LH1396, LH1539, MB082C, MB077B, } \\
\text { MB052B, MB434B, MB112C, } \\
\text { MB011B, MB543B, MB050B, } \\
\text { MB018B, MB027B, MB549C, LH989, } \\
\text { LH290. }\end{array}$ & $\begin{array}{l}\text { genetically blind hemizygous } \\
\text { male flies expressing Chrimson } \\
\text { under various GAL4 or split-GAL4 } \\
\text { drivers on chromosomes II or III. } \\
\text { tsh-Gal80 included to suppress } \\
\text { VNC expression. }\end{array}$ & 3-10 days & $\begin{array}{l}\text { Fig. 2A-D, Fig. } \\
\text { S2A,B }\end{array}$ \\
\hline
\end{tabular}




\begin{tabular}{|c|c|c|c|}
\hline $\begin{array}{l}\text { UAS-TNT }_{5905} \text { X X-GAL4 or split-GAL4 } \\
\text { X-GAL4: LH1538, LH1396, LH1539, } \\
\text { MB077B, MB052B, MB112C or + for } \\
\text { parental control }\end{array}$ & $\begin{array}{l}\text { flies with MB/LH neurons } \\
\text { silenced }\end{array}$ & 3-10 days & Fig. S1C \\
\hline $\begin{array}{l}\text { +;UAS-GCaMP6f;UAS-tdTomato x X- } \\
\text { GAL4 } \\
\text { X-GAL4: LH1396, MB052B }\end{array}$ & $\begin{array}{l}\text { GCaMP6f and tdTOM expressed } \\
\text { in LHAd1b2 using the LH1396 } \\
\text { split GAL4 driver or in MBONs15- } \\
19 \text { using MB052B }\end{array}$ & 5-21 days & Fig. 2E, Fig. S2E \\
\hline $\begin{array}{l}\text { +;MB027B-GAL4AD/+;UAS- } \\
\text { 10xGFP/MB027B-GAL4DB }\end{array}$ & $\begin{array}{l}\text { expression of GFP in } \alpha \text { '3 MBONs } \\
\text { for electrophysiology }\end{array}$ & 1-3 days & Fig. 2F \\
\hline \multicolumn{4}{|l|}{ Figures $3 \& 4$} \\
\hline $\begin{array}{l}\text { UAS-myrGFP, QUAS-mtdTomato (3x } \\
\text { HA); trans-Tango x X-GAL4 } \\
\text { X-GAL4: LH1396, MB052B }\end{array}$ & $\begin{array}{l}\text { Trans-tango driven by LH1396 } \\
\text { and MB052B }\end{array}$ & $\begin{array}{l}10-20 \\
\text { days; } \\
\text { raised at } \\
19^{\circ} \mathrm{C}\end{array}$ & Fig. 3A \\
\hline $\begin{array}{l}\text { norpAss/y;tsh-Gal80;UAS-Chrimson- } \\
\text { mVenus x X-GAL4 or split-GAL4 } \\
\text { Dorsal Inputs: VT056792-GAL4, } \\
\text { VT002458-GAL4, 84C10-GAL4, } \\
\text { VT027955-GAL4, 58F01-GAL4, } \\
\text { 58F02-GAL4, 71A02- } \\
\text { GAL4,VT026663-GAL4, VT20633- } \\
\text { GAL4, VT004849-GAL4, 23E10- } \\
\text { GAL4, 26B11-GAL4, 28H10-GAL4, } \\
\text { 12D12-GAL4, 65C03-GAL4, 45D04- } \\
\text { GAL4, LH2392, 43D09-AD;65C03- } \\
\text { DB, 12D12-AD;VT027955-DB, } \\
\text { VT026663AD;65C03DB, } \\
\text { 12D12AD;65C03DB } \\
\text { Ventral Inputs: VT036875-GAL4, } \\
\text { 33E06-GAL4, VT056509-GAL4, } \\
\text { 72A04-GAL4, 78G09-GAL4, } \\
\text { vt049652-GAL4, 13B10-GAL4, } \\
\text { VT033929-GAL4, VT046276-GAL4, } \\
\text { VT041421-GAL4,VT029515-GAL4, } \\
\text { VT041421AD;28H10DB, } \\
\text { VT041421AD;33E06DB, } \\
\text { VT041421DB;65H10DB, } \\
\text { VT029515AD; 78G09DB, LH1478, } \\
\text { 13B10AD;VT029515DB, 13B10AD; } \\
\text { VT041421DB }\end{array}$ & $\begin{array}{l}\text { genetically blind hemizygous } \\
\text { male flies expressing Chrimson } \\
\text { under various GAL4 or split-GAL4 } \\
\text { drivers on chromosomes II or III. } \\
\text { tsh-Gal80 included to suppress } \\
\text { VNC expression. }\end{array}$ & 3-10 days & Fig. 3B, Fig. S3 \\
\hline
\end{tabular}




\begin{tabular}{|c|c|c|c|}
\hline $\begin{array}{l}\text { Additional Cx neurons: 19C06-GAL4, } \\
\text { 65H10-GAL4, VT024599-GAL4, } \\
\text { VT058487-GAL4, VT000986-GAL4, } \\
\text { 24E05-GAL4, VT062617-GAL4, } \\
\text { 43D09-GAL4, VT019352-GAL4, } \\
\text { VT030322-GAL4, 94G02-GAL4, } \\
\text { VT050238-GAL4, VT063191-GAL4, } \\
\text { VT060736-GAL4, } \\
\text { 43D09AD;VT062617DB, 44B10- } \\
\text { GAL4; VT039497-GAL4,16D01-GAL4, } \\
\text { 67B06-GAL4, SS52577, SS52244, } \\
\text { SS54549, SS02255, SS00239, } \\
\text { 43D09AD; VT000986DB, VT063948- } \\
\text { GAL4, } \\
\text { 46G06-GAL4, V000624-GAL4, } \\
\text { VT060202-GAL4, 73A06-GAL4, } \\
\text { VT037489-GAL4, VT020739-GAL4, } \\
\text { VT032906-GAL4, 60D05-GAL4, } \\
\text { SS50464, SS02718, SS50420 }\end{array}$ & & & \\
\hline $\begin{array}{l}\text { CLIN x UAS-Chrimson- } \\
\text { mVenus;21D07-GAL4 }\end{array}$ & $\begin{array}{l}\text { 21D07>Chrimson-mVenus } \\
\text { filtered through CLIN to restrict } \\
\text { expression to type II (Cx) neurons }\end{array}$ & 3-10 days & $\begin{array}{l}\text { Fig. 3C anatomy } \\
\text { and behavior, Fig. } \\
\text { S4F }\end{array}$ \\
\hline $\begin{array}{l}\text { +;UAS-GCaMP6f;UAS-tdTomato x X- } \\
\text { GAL4 } \\
\text { X-GAL4: 21D07-GAL4, 65C03-GAL4, } \\
\text { vFB split (13B10AD;VT041421DB), } \\
\text { VT062617-GAL4 }\end{array}$ & $\begin{array}{l}\text { GCaMP6f and tdTOM expressed } \\
\text { under the various FB drivers }\end{array}$ & 5-21 days & $\begin{array}{l}\text { Fig, 3C,D,E imaging, } \\
\text { Fig, } 4 \text { D,E,F,G,H, } \\
\text { Fig. S4C }\end{array}$ \\
\hline $\begin{array}{l}\text { norpAsø/y;tsh-Gal80;UAS-Chrimson- } \\
\text { mVenus x X-GAL4 or split-GAL4 } \\
\text { X-GAL4: 65C03-GAL4, vFB split } \\
\text { (13B10AD;VT041421DB), VT062617- } \\
\text { GAL4, VT029515-GAL4, h } \Delta \text { C split1 } \\
\text { (19G02AD;VT062617DB), h } \Delta \text { C split2 } \\
\text { (VT024634AD; } \\
\text { VT062617DB) }\end{array}$ & $\begin{array}{l}\text { genetically blind hemizygous } \\
\text { male flies expressing Chrimson } \\
\text { under various GAL4 or split-GAL4 } \\
\text { drivers on chromosomes II or III. } \\
\text { tsh-Gal80 included to suppress } \\
\text { VNC expression. }\end{array}$ & & $\begin{array}{l}\text { Fig. 3D,E behavior, } \\
\text { Fig. 4I, Fig. S4F, G, } \\
\text { J }\end{array}$ \\
\hline $\begin{array}{l}\text { UAS(FRT.stop)-Chrimson- } \\
\text { mVenus; LexAop-FLP; ChAT-LexA; } \\
\text { ChAT-LexA x X-GAL4 or split-GAL4 } \\
\text { X-GAL4: 21D07-GAL4, 65C03-GAL4, } \\
\text { VT062617-GAL4, vFB split } \\
\text { (13B10AD;VT041421DB) }\end{array}$ & $\begin{array}{l}\text { cross to identify cholinergic } \\
\text { neurons within a GAL4 or split- } \\
\text { GAL4 line }\end{array}$ & 3-20 days & Fig. S4E \\
\hline
\end{tabular}




\begin{tabular}{|c|c|c|c|}
\hline $\begin{array}{l}\text { UAS(FRT.stop)-Chrimson- } \\
\text { mVenus; LexAop-FLP; Gad1-LexA x } \\
\text { X-GAL4 or split-GAL4 } \\
\text { X-GAL4: 65C03-GAL4, VT062617- } \\
\text { GAL4 }\end{array}$ & $\begin{array}{l}\text { cross to identify GABAergic } \\
\text { neurons within a GAL4 or split- } \\
\text { GAL4 line }\end{array}$ & 3-20 days & Fig. S4E \\
\hline $\begin{array}{l}\text { UAS-TNT }_{5005} \times \text { X-GAL4 or split-GAL4 } \\
\text { X-GAL4: 65C03-GAL4, 45D04-GAL4, } \\
\text { VT029515-GAL4, vFB split } \\
\text { (13B10AD;VT041421DB) }\end{array}$ & flies with FB neurons silenced & 3-10 days & Fig. S4I \\
\hline $\begin{array}{l}\text { norpA }{ }^{36 ;+;++} \text { flies backcrossed into the } \\
\text { w118 (\#5905) stock }\end{array}$ & norpA $^{36(5905)}$ & 3-10 days & Fig. S4H \\
\hline
\end{tabular}

\section{BEHAVIORAL EXPERIMENTS}

For behavioral experiments we used a modified version of the miniature wind tunnel setup described in (8). Briefly, flies were constrained to walk in a shallow arena with constant laminar airflow at $11.9 \mathrm{~cm} / \mathrm{s}$. In experiments with odor a $10 \mathrm{~s}$ pulse of $1 \%$ apple cider vinegar (Pastorelli) was introduced through solenoid valves located immediately below the arena. For optogenetic experiments, red LEDs (626nm, SuperBrightLEDS), interleaved with IR LEDS (850nm, Environmental Lights) in a lighting panel positioned $4.5 \mathrm{~cm}$ above the arena were illuminated using a BuckPuck (LEDDynamics, 1000mA, Digikey) and Arduino controller (teensy 2.0, PJRC). Light intensity was measured with a light meter (Thorlabs) and was $26 \mathrm{uW} / \mathrm{mm}$ for the majority of experiments. In flies expressing Chrimson using the CLIN technique the light level was $34 \mathrm{uW} / \mathrm{mm}$ (10) to compensate for low Chrimson expression in this line. Wind and odor stimuli were calibrated using a hotwire anemometer (Dantec Instruments) and photo-ionization detector (miniPID, Aurora Systems) respectively.

Flies (males or mated females) were collected 2 to 7 days before the experiment and housed in time shifted boxes on $12 \mathrm{~h}$ cycles. For optogenetic experiments, flies were placed on cornmeal-agar food supplemented with 50uL all-trans retinal (35mM stock: Sigma, R2500, dissolved in ethanol, stored at $-20^{\circ} \mathrm{C}$ ) mixed into $\sim 1$ teaspoon of hydrated potato flakes. These vials were covered with aluminum foil except for a small window near the fly plug. All flies began the experiment $(\sim 1.5-2 \mathrm{~h})$ between their subjective ZT1 and ZT4. 20-24h before the experiment we deprived flies of food by placing them in a polystyrene vial with damp shredded kimwipe. Flies were briefly anaesthetized by placing the vial on ice for $\sim 1 \mathrm{~min}$ before loading into the arena. Flies were allowed 5-10 minutes to recover before the experiment began. Flies for genetic silencing were run in the dark, while optogenetic activation experiments were run with ambient lighting. During experiments with odor, flies received blocks of three trial conditions in random order: wind and odor (30s wind alone, 10s odorized air, 30s wind alone), wind alone (70s), or no wind (70s). During optogenetic activation experiments flies received blocks of four trial conditions in random order: light stimulation with wind (30s wind, 10s wind and light, 30s wind), 
light stimulation alone (30s no stimulus, 10s light, 30s no stimulus), wind alone (70s wind), or blank trials $(70 s)$.

We analyzed behavioral data in a similar fashion as in (8). X,Y coordinates and orientation were tracked in real time at $50 \mathrm{~Hz}$ using custom Labview software (National Instruments). Data were further analyzed offline using custom Matlab scripts. Coordinates and orientation were low-pass filtered at $2.5 \mathrm{~Hz}$ using a 2-pole Butterworth filter. Any trials with tracking errors, or where the fly moved less than $25 \mathrm{~mm}$ overall were discarded from further analysis. Any fly which moved on less than 5 trials for a condition was excluded as well. For all measured parameters (see below) time periods when the fly was stationary (moving at less than $1 \mathrm{~mm} / \mathrm{s}$ ) were omitted. In trials with odor stimuli, we aligned the time courses of behavioral parameters to the actual time the fly encounter the odor based on their position is the arena, based on delays recorded by miniPID.

Behavioral parameters as a function of time were calculated as follows for each trial: Distance moved was calculated as the length of the hypotenuse between two pairs of coordinates at each frame. Groundspeed was calculated as the distance moved divided by the time interval of a frame (20ms). Upwind velocity was calculated as the change in Y-coordinates divided by the frame interval. Angular velocity was calculated as the absolute value of the change in unwrapped orientation divided by the time frame interval. Curvature was calculated as the filtered angular velocity divided by the filtered groundspeed. Probability of movement (pmove) was calculate by binarizing groundspeed with a threshold of $1 \mathrm{~mm} / \mathrm{s}$.

Quantification of average behavioral parameters represent average parameters across trials for each fly. All parameters were compared to a baseline period (10-25s in the trial) expect place preference where the baseline was taken from 25-30s into the trial (immediately before stimulus onset at 30s). We used the following time windows for analysis: probability of movement (pmove): $0-5 \mathrm{~s}$ from stimulus onset, upwind velocity (upwind): $0-5$ s from stimulus onset for periphery, $\mathrm{LH}, \mathrm{MB}, 0-10$ s from stimulus onset for FB, offset upwind velocity (upwindoff): 0-2s after stimulus offset, groundspeed: $2 \mathrm{~s}-5 \mathrm{~s}$ from stimulus onset, offset groundspeed: $0-2 \mathrm{~s}$ after stimulus offset, angular velocity (angv): $2 \mathrm{~s}-5 \mathrm{~s}$ from stimulus onset, offset angular velocity (angvoff): 0-2s after stimulus offset, onset angular velocity (angvon): $0-1$ s from stimulus onset, curavture: $2 \mathrm{~s}-5 \mathrm{~s}$ from stimulus onset, offset curvature (curvatureoff): $0-2 \mathrm{~s}$ after stimulus offset, onset curvature (curvature on): 0-1s from stimulus onset, place preference (placepref): $7.5 \mathrm{~s}$ from stimulus onset to $2.5 \mathrm{~s}$ after stimulus offset. For display purposes we do not depict the significance values in tables (Fig 3. B and Fig. S4) for upwind velocity for small magnitude increases $(<1 \mathrm{~mm} / \mathrm{s})$. We do not display values for curvature (both stimulation and offset) if angular velocity is below $<50 \mathrm{deg} / \mathrm{s}$ and groundspeed is $<4 \mathrm{~mm} / \mathrm{s}$. We excluded these values because the curvature increase was due to mostly to the drop in groundspeed and trajectories in these cases did not exhibit characteristics of search.

\section{CALCIUM IMAGING}


For calcium imaging experiments, flies were cold-anaesthetized and mounted in a version of the fly holder described in (29). The fly's head was positioned in a keyhole shaped metal cutout (etchit) within a plastic holder. We attached the fly to the holder using UV glue (Riverruns UV clear Glue, thick formula), and stabilized the proboscis to the head/body, leaving the antenna free to move. We removed the front two legs to prevent interference with airflow stimuli. Under Drosophila extracellular saline (103 $\mathrm{mM} \mathrm{NaCl}, 3 \mathrm{mM} \mathrm{KCl}, 5 \mathrm{mM}$ TES, $8 \mathrm{mM}$ trehalose dihydrate, $10 \mathrm{mM}$ glucose, $26 \mathrm{mM}$ NaHCO3, $1 \mathrm{mM}$ $\mathrm{NaH} 2 \mathrm{PO} 4 \mathrm{H} 20,1.5 \mathrm{mM} \mathrm{CaCl} 22 \mathrm{H} 2 \mathrm{O}$, and $4 \mathrm{mM} \mathrm{MgCl} 26 \mathrm{H} 2 \mathrm{O}, \mathrm{pH}$ 7.1-7.4, osmolarity 270-274 mOsm), we dissected away the cuticle at the back of fly's head using fine forceps. We removed the trachea, airsacs, and muscle over the surface of the brain. Flies were starved for 18-24h prior to the experiment. External saline bubbled with carbogen $\left(5 \% \mathrm{CO}_{2}, 95 \% \mathrm{O}_{2}\right)$ was perfused for the duration of the experiment.

2-Photon imaging was performed using a pulse infrared laster (Mai Tai DeepSea, Spectraphysics) with a Bergamo II microscope (Thorlabs). Images were acquired through a 20x water immersion objective (Olympus XLUMPLFLN 20x) using Thorlmage 3.0 software. The wavelength of the laser was set to 920 $\mathrm{nm}$ and power at the sample ranged from [13 to $66 \mathrm{~mW}$ ]. Spectral separation of emitted photons was accomplished with two bandpass filters (red, tdTOM, 607/70nm, green, GCamp6f, 525/50nm) and detected by GaAsP PMTs. Imaging areas varied depending on the genotype but were between $47 \mathrm{x}$ $47 \mathrm{uM}$ and $122 \times 74 \mathrm{uM}$. Imaging regions were identified first using the tdTOM signal under epifluorescence. Images were acquired at $\sim 5.0$ frames per second. Across genotypes we excluded any flies where we were unable to obtain 5 trials of each direction, either due to fat migration or cell death. We excluded 2 flies from the 65C03-GAL4 data which showed rhythmic spike like activity and did not respond to any phase of our stimulus. For VT062617-GAL4 imaging we excluded 1/17 flies as no columns showed had an average response $>2$ STD above baseline.

Airflow and odor stimuli were delivered using a 5-direction manifold described in $(28,20)$. Air was charcoal filtered, then passed through a flowmeter (Cole-Parmer), and proportional valves (EVP series, EV-05-0905; Clippard instruments laboratory,Cincinnati, $\mathrm{OH}$ ) to direct air or odorized air at the fly from one of 5 directions. Airspeed was $\sim 25 \mathrm{~cm} / \mathrm{s}$. We used a hotwire anemometer (Dantec Instuments) to verify that airspeed was equivalent across directions and constant through the wind and odor phases of the stimulus. Odorant (apple cider vinegar, 10\%) was diluted in distilled water on the day of the experiment. Stimuli acquisition consisted of $5 \mathrm{~s}$ without stimuli, $10 \mathrm{~s}$ of wind alone, 10 s of odorized wind, 10 s of wind alone and 8-10s of no stimulus following the wind. We randomized the direction odor was presented from in blocks of 5 trials and completed 5 blocks for each fly ( 25 total trials). We adjusted imaging position, Z-plane, gain, and power levels after each block as necessary. Of the flies included we present data from all 25 trials here.

Analysis of calcium data was performed as in (20). We used the CalmAn Matlab package, ImageJ, and custom Matlab scripts to align and analyze data. We used the CalmAn package (56) to implement the NoRMCorre rigid motion correction algorithm (57) on the red (tdTOM) time series and applied the same shifts to the green (GCaMP6f) times series. We drew regions of interest (ROIs) by hand on maximum intensity projections of the tdTom time series for the first trial. ROls were applied to all trials, and 
adjusted by hand using imageJ if significant drift occurred between trials. ROls were drawn around the following regions: for $\mathrm{LH} 1396$ the $\mathrm{ROI}$ was placed around the dendritic processes in the $\mathrm{LH}$, for MB052B the ROI was placed around the putative axonal processes in the protocerebrum. ROI location and imaging region was selected based on pilot experiments recording from different planes and ROls. For tangential FB inputs (FB5AB, 65C03-GAL4, and vFB split) we imaged from the FB layer innervated by each GAL4 line. We report imaging quantifications across the entire layer in the figures. In tangential inputs we did not observe obvious direction specific responses in different anatomical locations of the output layer. For VT062617-GAL4 imaging of h $\Delta \mathrm{C}$ neurons we drew ROIs across 8 putative columns of the FB based on the glomerular structure that was observable in the tdTom signal. In some cases the true number of columns was unclear and depended on the exact plane of imaging and positioning of the fly's head. ImageJ ROls were imported into Matlab using ReadlmageJROI (58). We calculated $\Delta F / F$ for the GCaMP6f time series by dividing the time series by the average fluorescence of the baseline period (first $5 \mathrm{~s}$ of the trial, excluding the first sample due to shutter lag). For main text figures we present the average $\Delta F / F$ signal for individual flies. Mean traces were calculated by resampling to 5 samples per second if frame rate varied between experiments. Supplemental heat maps were normalized to maximum response across all trials within an individual fly.

\section{ELECTROPHYSIOLOGY}

We performed whole cell patch clamp recordings as described previously $(20,28)$. Mounting and dissection were similar to that described above for calcium imaging except that we used hot wax rather than UV glue to fix the fly in place. In addition, we removed the sheath covering the brain using collagenase (5\% in extracellular saline, Worthington Biochemical Corporation Collagenase Type 4) under positive pressure applied with a fine tipped electrode (5-10uM diameter). Cell bodies of interest were visualized with 10x cytoplasmic GFP using an LED source (Cairn Research MONOLED) and filter cube (U-N19002 AT-GFP/F LP C164404). Brains were visualized under 40x magnification (Olympus, LUMPLFLN40XW) using a camera (Dage-MTI, IR-1000) and an LCD monitor (Samsung, SMT-1734). Cell bodies were cleaned using external saline and positive pressure, as well as light negative pressure to remove cell bodies near our cell of interest.

For whole-cell patch clamp recordings we pulled 6 to $10 \mathrm{M}$-Ohm glass pipettes made of thick walled glass (World Precision Instruments 1B150F-3) using a Sutter Instruments P-1000 puller. Pipettes were polished using a pressurized micro-forge (Scientific Instruments, CPM-2). Our intracellular solution contained $140 \mathrm{mM} \mathrm{KOH}, 140 \mathrm{mM}$ aspartic acid, $10 \mathrm{mM}$ HEPES, 1 mM EGTA, 1 $\mathrm{mM} \mathrm{KCl}, 4 \mathrm{mM} \mathrm{MgATP}, 0.5 \mathrm{mM}$ Na3GFP, and $13 \mathrm{mM}$ biocytin hydrazide (for visualization of neural processes). Current and voltage signals were amplified using either an A-M systems Model 2400 amplifier or a Molecular Devices Multiclamp 700B. Recordings acquired with the A-M systems amplifier were paired with additional preampfification using a Brownlee Precision 410 preamplifier. We controlled stimuli and hardware using custom Matlab and Arduino software scripts. All electrophysiological recordings were acquired at $10 \mathrm{kHz}$. 
Stimuli were delivered using an olfactometer similar to the one described in (59). Charcoalfiltered air was passed through a flowmeter (Cole-Parmer, $0.3 \mathrm{~L} / \mathrm{min}$ ), and then split into two airstreams that passed over either odorant $(10 \% \mathrm{ACV})$ or water. These two airstreams were then passed through two three-way solenoid valves (LFAA1201610H, Lee company) that allowed a signal to switch which airstream (odor or water) was directed into the main airflow. Main airflow $(1 \mathrm{~L} / \mathrm{min})$ was delivered to the fly through a teflon tube (4mm outer diameter, $2.5 \mathrm{~mm}$ inner diameter). The teflon tube was positioned $<1 \mathrm{~mm}$ from the head of the fly using a micro manipulator for each experiment using two cameras (Unibrain). The airflow delivery system was positioned on the right side of the fly, thus cells on the fly's right were ipsilateral while those on the left were contralateral. Pulses of $2 \mathrm{~s}, 10 \mathrm{~s}$ or $20 \mathrm{~s}$ of odor were presented to the fly, only 10 s of ACV is displayed here.

We used custom Matlab scripts to analyze electrophysiology data. To analyze membrane potential, we applied a $2.5 \mathrm{~Hz}$ Butterworth filter to remove spikes. We averaged the baseline period of of $2 s$ and subtracted this from the average time course for each fly for presentation purposes. We report the difference between the baseline period (wind only) and the average during the first $4 \mathrm{~s}$ of the odor period. The resting potential varied between (-34.6 $\mathrm{mV}$ and $-25.2 \mathrm{mV}$ for MBONs a'3). The average resting potential was: $-30.8 \mathrm{mV}$. We recorded from a total of $12 \mathrm{MBONs}, 6$ on each side that met our criteria for quality of recording based on input to access ratio great than $5: 1$.

\section{IMMUNOHISTOCHEMISTRY}

We performed immunohistochemistry as in previous reports $(20,28)$. We fixed brains for 15 minutes in $4 \%$ paraformaldehyde (in 1X phosphate buffered saline, PBS). Next we washed the brain three times in PBS and stored at $4^{\circ} \mathrm{C}$ until antibody staining (immediately or within 2 weeks). We incubated brains in a blocking solution containing 5\% normal goat serum dissolved in PBST (1x PBS with $0.2 \%$ Triton-X) for 20-60 minutes. Brains were incubated at room temperature in a solution of primary antibodies (see below for exact components). We then washed brains three times in PBST and incubated brains in a secondary antibody solution at room temperature for $24 \mathrm{~h}$. We washed brains three times in PBST and then stored in PBS at $4^{\circ} \mathrm{C}$ until imaging. To mount brains for imaging we placed brains in vectashield (Vector Labs $\mathrm{H}-1000$ ) and sealed with coverslips and nail polish. We imaged brains at 20x magnification of a Zeiss LSM 800 confocal microscope with a 20x objective (Zeiss W Plan-Apochromat 20x/1.0 DIC CG 0.17 M27 75mm). All brains were imaged at 1-1.25uM depth resolution. Final images are presented as maximum $Z$ projections over relevant depths.

To visualize the expression of Chrimson-mVenus in driver lines used in optogenetic activation experiments we dissected brains of females from the same cross as experimental males. We visualized 1-3 brains for each genotype (data not shown) to assess the breadth of expression under the Chrimson effector. 
We used the following antibody mixes for the experiments listed: Chrimson/neurotransmitter stains: primary: chicken anti-GFP (1:50) \& mouse anti-nc82 (1:50); secondary: anti-chicken Alexa488 (1:250) \& anti-mouse Alexa 633 (1:250). Electrophysiology stains: primary: chicken anti-GFP (1:50) \& mouse anti-nc82 (1:50); secondary: anti-chicken Alexa488 (1:250), anti-mouse Alexa 633 (1:250) and Alexa568-conjugated streptavidin (1:1000). Trans-tango and CLIN stains: primary: chicken anti-GFP (1:50), mouse anti-nc82 (1:50), \& rabbit anti-dsred (1:500); secondary: anti-chicken Alexa 488 (1:250), anti-mouse Alexa 633 (1:250) and anti-rabbit Alexa 568 (1:250). GABA stains: chicken anti-GFP (1:50), mouse anti-nc82 (1:50) \& rabbit anti-GABA (1:100); anti chicken Alexa488 (1:250), anti-mouse Alexa 633 (1:250) \& anti-rabbit Alexa 568 (1:250).

\section{QUANTIFICATION AND STATISTICAL ANALYSIS}

Behavior: Based on previous analysis of behavioral data $(8,28)$ we assumed behavioral data was not normally distributed. We applied non-parametric statistics to compare results and corrected for multiple comparisons using the Bonferroni method. We used the Wilcoxon signed rank test (Matlab's signrank) to compare the average baseline value of each parameter to the average of the parameter over a window of interest. Between genotype comparisons (genetic silencing experiments) were made between baseline subtracted parameter values using the Mann-Whitney $U$ test (Matlab's ranksum). For behavioral time courses we display standard error around the mean. For summary plots we present standard deviation around the mean. Bonferroni corrections were applied based on the number of genotypes tested labelling similar neuron types (i.e. 6 in Fig. 1D, 22 for all dorsal FB inputs in Dig. 3C) or for the number of comparisons made to control in genetic silencing experiments ( 3 in Fig. 1F).

Imaging and physiology: We used parametric tests for calcium imaging and electrophysiological data. When testing for significant differences in odor responses across directions we averaged individual trials across flies, and performed an ANOVA across pooled trials across flies. When assessing differences between wind onset responses and odor responses we calculated mean wind and odor responses in 5s windows following onset. We compared wind and odor periods using a paired student's t-test (Fig. S 4D). Similarly we performed paired student's t-test when comparing wind and odor responses in MBON electrophysiology experiments and an unpaired student's t-test when comparing odor responses between ipsilateral and contralateral MBONs.

\section{CONNECTOMIC ANALYSIS}

Data from the hemibrain connectome (17) were interpreted using neuprint explorer (neuprint.janelia.org, version d2a8f5785d73421096f7cdc09ad585e5). Further analysis and visualization was completed using custom Matlab and Python scripts. For the analysis shown in Fig. 4B, the location of $F B 5 A B$ synapses onto $h \triangle C$ was determined by their $\mathrm{x}$ position. No filtering or constraints were applied in synapse counts for this panel. For the analysis in Supp. Fig. 4B, we counted all FB tangential neurons two synapses downstream of the following projection neurons: VM7d_adPN, 
VM7v_adPN, DM1_IPN, DM4_adPN, DM4_vPN, VA2_adPN, DP1I_adPN, DP1I_vPN, DL2d_adPN, DL2d_vPN, DL2v_adPN, DC4_adPN, DC4_vPN, DP1m_adPN, DP1m_vPN in which the intermediate neurons contained LH (they were lateral horn neurons) and where synaptic weights exceeded a weight of 3 .

\section{MODELING}

FB heading bumps were simulated in windPFN, PFL3, and PFL2 populations using one-cycle sinusoids defined by:

$$
\text { activity }=\cos \left(F B_{\text {location }}-\left(180^{\circ}-\text { heading }+ \text { shift }\right)\right)^{\star} \text { scale }
$$

Where $F B_{\text {location }}$ refers to the spatial location within the $\mathrm{FB}\left(0^{\circ}=\right.$ left $\mathrm{FB}, 360^{\circ}=$ right $\left.\mathrm{FB}\right)$, heading refers to the heading direction of the fly, shift refers to the phase shift introduced by a given cell type, and scale refers to the sinusoid amplitude. The shift parameters were taken directly from the connectome (16), and were defined as the following: shift left_PFN $_{1}=+45^{\circ}$, shift right_PFN $=-45^{\circ}$, shift feft_PFL3 $=-90^{\circ}$, shift right_PFL3 $=+90^{\circ}$, shift left_PFL2 $_{2}=-180$, shiftright_PFL3 $=+180^{\circ}$. The scale parameters were equal to 1 for all cell types except windPFNs, which instead varied with wind direction according to the following sinusoid equation:

$$
\text { scale }_{\text {windPFN }}=\sin \left((\text { heading }- \text { wind })+\left(90^{\circ}-\text { peak }\right)\right)
$$

Where heading refers to the heading direction of the fly, wind refers to the allocentric wind direction, and peak refers to the egocentric wind direction that maximally activates the PFN cell type (peak left_PFN $_{\text {. }}$ $=-45^{\circ}$, peak right_PFN $=+45^{\circ}$ ).

To predict turning via the direct and indirect pathways, the heading bumps in PFN populations were shifted ( $0^{\circ}$ for direct pathway, $180^{\circ}$ for indirect pathway), added to the heading bumps in left and right PFL3 populations, and then the amplitudes of the resulting sinusoids (one for left PFL3 and one for right PFL3) were compared:

$$
\begin{aligned}
& \% \text { RightPFL3 }{ }_{\text {dir }}=\max \left(P F L 3_{\text {right }}+P N_{\text {right }}+P F N_{\text {left }}\right) /\left[\max \left(P F L 3_{\text {right }}+P F N_{\text {right }}+P F N_{\text {left }}\right)+\max \left(P F L 3_{\text {left }}+\right.\right. \\
& \left.P F N_{\text {right }}+P F N_{\text {left }}\right]^{*} 100 \% \\
& \% \text { RightPFL3 }{ }_{\text {indir }}=\max \left(P F L 3_{\text {right }}-\left(P F N_{\text {right }}+P F N_{\text {left }}\right)\right) /\left[\max \left(P F L 3_{\text {right }}-\left(P F N_{\text {right }}+P F N_{\text {left }}\right)\right)+\max \left(P F L 3_{\text {left }}\right. \text { - }\right. \\
& \left.\left.\left(P F N_{\text {right }}+P^{P F} N_{\text {left }}\right)\right)\right]^{*} 100 \%
\end{aligned}
$$

To predict forward velocity via the direct and indirect pathways, the heading bumps in PFN populations were shifted ( $0^{\circ}$ for direct pathway, $180^{\circ}$ for indirect pathway), added to the heading bump in the left and right PFL2 population, and then the amplitude of the resulting sinusoid was computed. 
We doubled the amplitude to indicate that this process happens twice, once for left PFNs and once for right PFNs:

$$
\begin{gathered}
P F L 2_{\text {dir }}=2{ }^{*} \max \left(P F L 2+P F N_{\text {right }}+P F N_{\text {left }}\right) \\
P F L 2_{\text {indir }}=2{ }^{*} \max \left(P F L 2-\left(P F N_{\text {right }}+P F N_{\text {left }}\right)\right)
\end{gathered}
$$

\begin{tabular}{|c|c|c|}
\hline Resource & Source & Identifier \\
\hline \multicolumn{3}{|l|}{ antibodies } \\
\hline chicken anti-GFP & Fisher Scientific & RRID:AB_1074893 \\
\hline mouse anti-nc82 & DSHB & RRID:AB_2314866 \\
\hline rabbit anti-DsRed & Clontech & 632496 \\
\hline Rabbit anti-GABA & Sigma & RRID:AB_477652 \\
\hline $\begin{array}{l}\text { Alexa488-conjugated goat anti- } \\
\text { chicken }\end{array}$ & Fisher Scientific & RRID:AB_2534096 \\
\hline $\begin{array}{l}\text { Alexa633-conjugated goat anti- } \\
\text { mouse }\end{array}$ & Fisher Scientific & RRID:AB_2535719 \\
\hline $\begin{array}{l}\text { Alexa568-conjugated goat anti- } \\
\text { rabbit }\end{array}$ & Fisher Scientific & RRID:AB_2315774 \\
\hline $\begin{array}{l}\text { Alexa568-conjugated } \\
\text { streptavidin }\end{array}$ & Fisher Scientific & RRID:AB_2576217 \\
\hline \multicolumn{3}{|l|}{ chemicals } \\
\hline apple cider vinegar & Pastorelli & \\
\hline \multicolumn{3}{|l|}{$\begin{array}{l}\text { experimental models: } \\
\text { organisms/strains }\end{array}$} \\
\hline $\begin{array}{l}\text { D. mel: orco-GAL4 (backcrossed } \\
\text { into w118 5905) }\end{array}$ & BDSC & RRID:BDSC_26818 \\
\hline D. mel: OR59a-GAL4 (III) & $\begin{array}{l}\text { BDSC via Marc } \\
\text { Gershow }\end{array}$ & RRID:BDSC_9990 \\
\hline D. mel: OR42b-GAL4 (II) & $\begin{array}{l}\text { BDSC via Marc } \\
\text { Gershow }\end{array}$ & RRID:BDSC_9971 \\
\hline D. mel: OR42a-GAL4 (III) & BDSC & RRID:BDSC_9969 \\
\hline D. mel: IR75a-GAL4/TM6B & BDSC & RRID:BDSC_41748 \\
\hline D. mel: IR64a-GAL4;TM2/TM6 & BDSC & RRID:BDSC_41732 \\
\hline D. mel: OR92a-GAL4 & BDSC & RRID:BDSC_23139 \\
\hline D. mel: IR8a-GAL4;TM2/TM6 & BDSC & RRID:BDSC_41731 \\
\hline D. mel:60D05-GAL4 & BDSC & RRID:BDSC_39247 \\
\hline D. mel: 46G06-GAL4 & BDSC & RRID:BDSC_41271 \\
\hline D. mel: 44B10-GAL4 & BDSC & RRID:BDSC_50202 \\
\hline
\end{tabular}

KEY RESOURCES TABLE 


\begin{tabular}{|c|c|c|}
\hline D. mel: 16D01-GAL4 & BDSC & RRID:BDSC_48722 \\
\hline D. mel: 19C06-GAL4 & BDSC & RRID:BDSC_48843 \\
\hline D. mel: 67B06-GAL4/TM3 & BDSC & RRID:BDSC_48294 \\
\hline D. mel: 73A06-GAL4 & BDSC & RRID:BDSC_39805 \\
\hline D. mel: 84C10-GAL4 & BDSC & RRID:BDSC_48378 \\
\hline D. mel: 71A02-GAL4 & $\mathrm{BDSC}$ & RRID:BDSC_39560 \\
\hline D. mel:23E10-GAL4 & BDSC & RRID:BDSC_49032 \\
\hline D. mel:26B11-GAL4 & BDSC & RRID:BDSC_49164 \\
\hline D. mel:28H10-GAL4 & BDSC & RRID:BDSC_48085 \\
\hline D. mel: 12D12-GAL4 & BDSC & RRID:BDSC_48506 \\
\hline D. mel: 65C03-GAL4 & BDSC & RRID:BDSC_41290 \\
\hline D. mel: 45D04-GAL4 & BDSC & RRID:BDSC_48160 \\
\hline D. mel: 65H10-GAL4 & BDSC & RRID:BDSC_49614 \\
\hline D. mel: 43D09-GAL4 & BDSC & RRID:BDSC_49553 \\
\hline D. mel:24E05-GAL4 & BDSC & RRID:BDSC_49081 \\
\hline D. mel: 94G04-GAL4 & BDSC & RRID:BDSC_40699 \\
\hline D. mel: 33E06-GAL4 & BDSC & RRID:BDSC_48114 \\
\hline D. mel: 72A04-GAL4 & BDSC & RRID:BDSC_46665 \\
\hline D. mel: 78G09-GAL4 & BDSC & RRID:BDSC_40015 \\
\hline D. mel: 13B10-GAL4 & BDSC & RRID:BDSC_48548 \\
\hline D. mel:21D07-GAL4 & BDSC & RRID:BDSC_48943 \\
\hline D. mel: 58F01-GAL4 & BDSC & RRID:BDSC_48213 \\
\hline D. mel: 58F02-GAL4 & BDSC & RRID:BDSC_39186 \\
\hline D. mel: empty GAL4 & BDSC & RRID:BDSC_68384 \\
\hline D. mel: empty split GAL4 & BDSC & RRID:BDSC_79603 \\
\hline D. mel: 43D09AD & BDSC & RRID:BDSC_70691 \\
\hline D. mel: VT000986DB & BDSC & RRID:BDSC_75369 \\
\hline D. mel: VT026663AD & BDSC & RRID:BDSC_73054 \\
\hline D. mel: 12D12DB & BDSC & RRID:BDSC_69213 \\
\hline D. mel: VT027955DB & BDSC & RRID:BDSC_73229 \\
\hline D. mel: 65C03DB & BDSC & RRID:BDSC_69323 \\
\hline D. mel: 65C03AD & BDSC & RRID:BDSC_71005 \\
\hline D. mel: 45D04-DB & BDSC & RRID:BDSC_69239 \\
\hline D. mel: 12D12AD & BDSC & RRID:BDSC_70539 \\
\hline D. mel: VT041421AD & BDSC & RRID:BDSC_71457 \\
\hline D. mel: 28H10DB & BDSC & RRID:BDSC_69496 \\
\hline D. mel: 33E06DB & BDSC & RRID:BDSC_69229 \\
\hline D. mel: 65H10DB & BDSC & RRID:BDSC_86702 \\
\hline D. mel: VT029515AD & BDSC & RRID:BDSC_74286 \\
\hline D. mel: 78G09DB & BDSC & RRID:BDSC_69718 \\
\hline D. mel: 13B10AD & BDSC & RRID:BDSC_68828 \\
\hline D. mel: VT029515DB & BDSC & RRID:BDSC_75470 \\
\hline D. mel: VT041421DB & BDSC & RRID:BDSC_73472 \\
\hline
\end{tabular}




\begin{tabular}{|c|c|c|}
\hline D. mel: VT063948-GAL4 & VDRC & VDRC: 200657 \\
\hline D. mel: VT000624-GAL4 & VDRC & VDRC: 202720 \\
\hline D. mel: VT060202-GAL4 & VDRC & VDRC: 202779 \\
\hline D. mel: VT037489-GAL4 & VDRC & VDRC: 206278 \\
\hline D. mel: VT020739-GAL4 & VDRC & VDRC: 201501 \\
\hline D. mel: VT032906-GAL4 & VDRC & VDRC: 202537 \\
\hline D. mel: VT039497-GAL4 & VDRC & VDRC: 205730 \\
\hline D. mel: VT056792-GAL4 & VDRC & VDRC: 206056 \\
\hline D. mel: VT002458-GAL4 & VDRC & VDRC: 202329 \\
\hline D. mel: VT027955-GAL4 & VDRC & VDRC: 202476 \\
\hline D. mel: VT026663-GAL4 & VDRC & VDRC: 203208 \\
\hline D. mel: VT020633-GAL4 & VDRC & VDRC: 207839 \\
\hline D. mel: VT004849-GAL4 & VDRC & VDRC: 207295 \\
\hline D. mel: VT024599-GAL4 & VDRC & VDRC: 202132 \\
\hline D. mel: VT058487-GAL4 & VDRC & VDRC: 207739 \\
\hline D. mel: VT062617-GAL4 & VDRC & VDRC: 206875 \\
\hline D. mel: VT000986-GAL4 & VDRC & VDRC: 207036 \\
\hline D. mel: VT019352-GAL4 & VDRC & VDRC: 201718 \\
\hline D. mel: VT030322 & VDRC & VDRC: 204795 \\
\hline D. mel: VT050238-GAL4 & VDRC & VDRC: 201475 \\
\hline D. mel: VT063191-GAL4 & VDRC & VDRC: 205643 \\
\hline D. mel: VT060736-GAL4 & VDRC & VDRC: 202781 \\
\hline D. mel: VT036875-GAL4 & VDRC & VDRC: 203402 \\
\hline D. mel: VT056509-GAL4 & VDRC & VDRC: 201744 \\
\hline D. mel: VT049652-GAL4 & VDRC & VDRC: 204721 \\
\hline D. mel: VT033929-GAL4 & VDRC & VDRC: 206842 \\
\hline D. mel: VT046276-GAL4 & VDRC & VDRC: 207814 \\
\hline D. mel: VT041421-GAL4 & VDRC & VDRC: 200867 \\
\hline D. mel: VT029515-GAL4 & VDRC & VDRC: 204785 \\
\hline D. mel: LH1396 & Janelia & Janelia: 3026621 \\
\hline D. mel: LH1538 & Janelia & Janelia: 3024098 \\
\hline D. mel: LH1539 & Janelia & Janelia: 3024933 \\
\hline D. mel: LH989 & Janelia & Janelia: 3032170 \\
\hline D. mel: LH290 & Janelia & Janelia: 2502068 \\
\hline D. mel: LH2392/CyO;TM6 & Janelia & Janelia: 3024051 \\
\hline D. mel: LH1478/CyO;TM6 & Janelia & Janelia: 3032196 \\
\hline D. mel: MB052B & Karla Kaun & \\
\hline D. mel: MB077B & Karla Kaun & Janelia: 2135209 \\
\hline D. mel: MB543B & Karla Kaun & Janelia: 2501888 \\
\hline D. mel: MB050B0 & Karla Kaun & Janelia: 2135100 \\
\hline D. mel: MB018B/CyO & Karla Kaun & Janelia: 2135069 \\
\hline D. mel: MB027B & Karla Kaun & Janelia: 2135078 \\
\hline D. mel: MB549C/TM6b & Karla Kaun & Janelia: 2501897 \\
\hline
\end{tabular}




\begin{tabular}{|c|c|c|}
\hline D. mel: MB082C & Karla Kaun & Janelia: 2135130 \\
\hline D. mel: MB434B/CyO & Janelia & Janelia: 2501775 \\
\hline D. mel: MB112C & Janelia & Janelia: 2135209 \\
\hline D. mel: MB011B & Janelia & Janelia: 2135062 \\
\hline D. mel: SS52577 & Janelia & Janelia: 3028433 \\
\hline D. mel: SS02255/[TM6b] & Janelia & Janelia: 3018121 \\
\hline D. mel: SS52244/[CyO] & Janelia & Janelia: 3028329 \\
\hline D. mel: SS54549 & Janelia & Janelia: 3029433 \\
\hline D. mel: SS02239 & Janelia & Janelia: 3018105 \\
\hline D. mel: SS50464 & Janelia & Janelia: 3028081 \\
\hline D. mel: SS02718 & Janelia & Janelia: 3018714 \\
\hline D. mel: SS50420 & Janelia & Janelia: 3028108 \\
\hline $\begin{array}{l}\text { D. mel: orco-GAL4,IR8a- } \\
\text { GAL4/[CyO] }\end{array}$ & this study & \\
\hline D. mel: 43D09AD;VT000986DB & this study & \\
\hline D. mel: VT026663AD;12D12DB & this study & \\
\hline D. mel: VT026663AD;VT027955DB & this study & \\
\hline D. mel: 43D09AD;65C03DB & this study & \\
\hline D. mel: 65C03AD;45D04DB & this study & \\
\hline D. mel: 12D12AD;vt027955DB & this study & \\
\hline D. mel: VT026663AD;65C03DB & this study & \\
\hline D. mel: 12D12AD;65C03DB & this study & \\
\hline D. mel: VT041421AD;28H10DB & this study & \\
\hline D. mel: VT041421AD;33E06DB & this study & \\
\hline D. mel: VT041421AD;65H10DB & this study & \\
\hline D. mel: VT029515AD;78G09DB & this study & \\
\hline D. mel: 13B10AD;VT029515DB & this study & \\
\hline D. mel: 13B10AD;VT041421DB & this study & \\
\hline $\begin{array}{l}\text { D. mel: 20XUAS- } \\
\text { IVSCsChrimson.mVenus\}attP40 (II) }\end{array}$ & BDSC & RRID: BDSC_55135 \\
\hline $\begin{array}{l}\text { D. mel: 20x-UAS-IVS-csChrimson- } \\
\text { mVenus-attp2 (III) }\end{array}$ & BDSC & RRID: BDSC_55136 \\
\hline D. mel: UAS-tdTOM (III) & BDSC & RRID: BDSC_36328 \\
\hline $\begin{array}{l}\text { D. mel: } \\
\text { norpA^36;T(2,3)En^es/([cyo- } \\
\text { afgp;TM3-agfp]) }\end{array}$ & Rachel Wilson & \\
\hline $\begin{array}{l}\text { D. mel: +(HCS);+;P\{10xUAS-IVS- } \\
\text { Syn21-GFP-p10\}attP2 (10X UAS- } \\
\text { GFP) (Backcrossed) }\end{array}$ & Michael Dickinson & \\
\hline D. mel: UAS-GCaMP6f;UAS-tdTOM & Michael Dickinson & \\
\hline
\end{tabular}




\begin{tabular}{|c|c|c|}
\hline $\begin{array}{l}\text { D. mel: w[*]; P\{w[+mC] = UAS- } \\
\text { TeTxLC.tnt }\} E 2 \text { (UAS-TNTe) }\end{array}$ & Matthieu Louis & \\
\hline tsh-Gal80(w-)/CyO (y) & $\begin{array}{l}\text { Julie Simpson via } \\
\text { Richard Mann }\end{array}$ & \\
\hline $\begin{array}{l}\text { D. mel: UAS-myrGFP, QUAS- } \\
\text { mtdTomato (3x HA); } \\
\text { trans-Tango }\end{array}$ & Gilad Barnea & \\
\hline $\begin{array}{l}\text { D. mel: } \\
\text { UAS(FRT.stop)CsChrimson.mVenu } \\
\text { s/P\{hs-hid\}Y; LexAop-FLP/(CyO); } \\
\text { ChAT-LexA/TM6b }\end{array}$ & Matthew Clark & \\
\hline $\begin{array}{l}\text { D. mel: } \\
\text { UAS(FRT.stop)CsChrimson.mVenu } \\
\text { s/P\{hs-hid\}Y; LexAop-FLP; Gad1- } \\
\text { LexA/TM3 sb }\end{array}$ & Matthew Clark & \\
\hline $\begin{array}{l}\text { D. mel: CLIN: } \\
\text { dpn(KDRT.stop)cre.PEST ; } \\
\text { Act(loxP.GAL80.stop)lexA::p65, } \\
\text { lexAop-rCD2::RFP,UAS-mCD8:: } \\
\text { GFP-p10; Stg14KD/TM6B, Tb[+] }\end{array}$ & Mubarak Syed & RRID:BDSC_67094 and RRID:BDSC_67089 \\
\hline $\begin{array}{l}\text { D. mel: norpA^36;UAS- } \\
\text { Chrimson/[CyO.a-GFP];10x UAS- } \\
\text { GFP }\end{array}$ & this study & \\
\hline $\begin{array}{l}\text { D. mel: norpA^36;tsh-Gal80/cyo- } \\
\text { agfp;UAS-Chrimson-mVenus }\end{array}$ & this study & \\
\hline $\begin{array}{l}\text { D. mel: norpA^36 (backcrossed in } \\
\text { w118) }\end{array}$ & [8] & \\
\hline \multicolumn{3}{|l|}{ software/algorithms/datsets } \\
\hline Matlab & & $\begin{array}{l}\text { https://www.mathworks.com/products/matlab.ht } \\
\mathrm{ml}\end{array}$ \\
\hline ImageJ/FIJI & & https://imagej.net/Fiji \\
\hline miniature windtunnes & [8] & https://doi.org/10.5061/dryad.g27mq71 \\
\hline neuprint hemibrain version 1.2 & {$[17]$} & $\begin{array}{l}\text { https://neuprint.janelia.org/?dataset=hemibrain\% } \\
\text { 3Av1.2\&qt=findneurons }\end{array}$ \\
\hline
\end{tabular}




\section{References}

1. Sarel, A., Finkelstein, A., Las, L. and Ulanovsky, N., 2017. Vectorial representation of spatial goals in the hippocampus of bats. Science, 355(6321), pp.176-180.

2. Butler, W.N., Hardcastle, K. and Giocomo, L.M., 2019. Remembered reward locations restructure entorhinal spatial maps. Science, 363(6434), pp.1447-1452.

3. Murlis, J., Elkinton, J.S. and Carde, R.T., 1992. Odor plumes and how insects use them. Annual review of entomology, 37(1), pp.505-532.

4. Cardé, R.T. and Willis, M.A., 2008. Navigational strategies used by insects to find distant, windborne sources of odor. Journal of chemical ecology, 34(7), pp.854-866.

5. Page, J.L., Dickman, B.D., Webster, D.R. and Weissburg, M.J., 2011. Getting ahead: contextdependent responses to odorant filaments drive along-stream progress during odor tracking in blue crabs. Journal of Experimental Biology, 214(9), pp.1498-1512.

6. Wolf, H. and Wehner, R., 2000. Pinpointing food sources: olfactory and anemotactic orientation in desert ants, Cataglyphis fortis. Journal of Experimental Biology, 203(5), pp.857-868.

7. Mech, L.D., 1966. The Wolves of isle royale (No. 7). US Government Printing Office.

8. Álvarez-Salvado, E., Licata, A.M., Connor, E.G., McHugh, M.K., King, B.M., Stavropoulos, N., Victor, J.D., Crimaldi, J.P. and Nagel, K.I., 2018. Elementary sensory-motor transformations underlying olfactory navigation in walking fruit-flies. Elife, 7, p.e37815.

9. Demir, M., Kadakia, N., Anderson, H.D., Clark, D.A. and Emonet, T., 2020. Walking Drosophila navigate complex plumes using stochastic decisions biased by the timing of odor encounters. Elife, 9, p.e57524.

10. Aso, Y., Sitaraman, D., Ichinose, T., Kaun, K.R. and Vogt, K., 2014. Belliart-Gué rin, G., Plaçais, P. Y., Robie, AA, Yamagata, N., Schnaitmann, C., et al.(2014b). Mushroom body output neurons encode valence and guide memory-based action selection in Drosophila. eLife, 3, p.e04580.

11. Cognigni, P., Felsenberg, J. and Waddell, S., 2018. Do the right thing: neural network mechanisms of memory formation, expression and update in Drosophila. Current opinion in neurobiology, 49, pp.51-58.

12. Ardin, P., Peng, F., Mangan, M., Lagogiannis, K. and Webb, B., 2016. Using an insect mushroom body circuit to encode route memory in complex natural environments. PLoS computational biology, 12(2), p.e1004683.

13. Collett, M. and Collett, T.S., 2018. How does the insect central complex use mushroom body output for steering?. Current Biology, 28(13), pp.R733-R734.

14. Sun, X., Yue, S. and Mangan, M., 2020. A decentralised neural model explaining optimal integration of navigational strategies in insects. Elife, 9, p.e54026.

15. Honkanen, A., Adden, A., da Silva Freitas, J. and Heinze, S., 2019. The insect central complex and the neural basis of navigational strategies. Journal of Experimental Biology, 222(Suppl 1).

16. Hulse, B.K., Haberkern, H., Franconville, R., Turner-Evans, D.B., Takemura, S., Wolff, T., Noorman, M., Dreher, M., Dan, C., Parekh, R. and Hermundstad, A.M., 2020. A connectome of the Drosophila central complex reveals network motifs suitable for flexible navigation and contextdependent action selection. bioRxiv. 
17. Scheffer, L.K., Xu, C.S., Januszewski, M., Lu, Z., Takemura, S.Y., Hayworth, K.J., Huang, G.B., Shinomiya, K., Maitlin-Shepard, J., Berg, S. and Clements, J., 2020. A connectome and analysis of the adult Drosophila central brain. Elife, 9, p.e57443.

18. Li, F., Lindsey, J.W., Marin, E.C., Otto, N., Dreher, M., Dempsey, G., Stark, I., Bates, A.S., Pleijzier, M.W., Schlegel, P. and Nern, A., 2020. The connectome of the adult Drosophila mushroom body provides insights into function. Elife, 9, p.e62576.

19. Schlegel, P., Bates, A.S., Stürner, T., Jagannathan, S., Drummond, N., Hsu, J., Capdevila, L.S., Javier, A., Marin, E.C., Barth-Maron, A. and Tamimi, I.F., 2020. Information flow, cell types and stereotypy in a full olfactory connectome. bioRxiv.

20. Currier, T.A., Matheson, A.M.M. and Nagel, K.I., 2020. Encoding and control of orientation to airflow by a set of Drosophila fan-shaped body neurons. Elife, 9, p.e61510.

21. Lyu, C., Abbott, L.F. and Maimon, G., 2020. A neuronal circuit for vector computation builds an allocentric traveling-direction signal in the Drosophila fan-shaped body. bioRxiv.

22. Lu, J., Westeinde, E.A., Hamburg, L., Dawson, P.M., Lyu, C., Maimon, G., Druckmann, S. and Wilson, R.I., 2020. Transforming representations of movement from body-to world-centric space. bioRxiv.

23. Larsson, M.C., Domingos, A.I., Jones, W.D., Chiappe, M.E., Amrein, H. and Vosshall, L.B., 2004. Or83b encodes a broadly expressed odorant receptor essential for Drosophila olfaction. Neuron, 43(5), pp.703-714.

24. Silbering, A.F., Rytz, R., Grosjean, Y., Abuin, L., Ramdya, P., Jefferis, G.S. and Benton, R., 2011. Complementary function and integrated wiring of the evolutionarily distinct Drosophila olfactory subsystems. Journal of Neuroscience, 31(38), pp.13357-13375.

25. Jung, S.H., Hueston, C. and Bhandawat, V., 2015. Odor-identity dependent motor programs underlie behavioral responses to odors. Elife, 4, p.e11092.

26. Dolan, M.J., Frechter, S., Bates, A.S., Dan, C., Huoviala, P., Roberts, R.J., Schlegel, P., Dhawan, S., Tabano, R., Dionne, H. and Christoforou, C., 2019. Neurogenetic dissection of the Drosophila lateral horn reveals major outputs, diverse behavioural functions, and interactions with the mushroom body. Elife, 8, p.e43079.

27. Bates, A.S., Schlegel, P., Roberts, R.J., Drummond, N., Tamimi, I.F., Turnbull, R., Zhao, X., Marin, E.C., Popovici, P.D., Dhawan, S. and Jamasb, A., 2020. Complete connectomic reconstruction of olfactory projection neurons in the fly brain. Current Biology, 30(16), pp.3183-3199.

28. Schulze, A., Gomez-Marin, A., Rajendran, V.G., Lott, G., Musy, M., Ahammad, P., Deogade, A., Sharpe, J., Riedl, J., Jarriault, D. and Trautman, E.T., 2015. Dynamical feature extraction at the sensory periphery guides chemotaxis. Elife, 4, p.e06694.

29. Suver, M.P., Matheson, A.M.M., Sarkar, S., Damiata, M., Schoppik, D. and Nagel, K.I., 2019. Encoding of wind direction by central neurons in Drosophila. Neuron, 102(4), pp.828-842.

30. Mamiya, A., Beshel, J., Xu, C. and Zhong, Y., 2008. Neural representations of airflow in Drosophila mushroom body. PLoS One, 3(12), p.e4063.

31. Scaplen, K.M., Talay, M., Fisher, J.D., Cohn, R., Sorkaç, A., Aso, Y., Barnea, G. and Kaun, K.R., 2021. Transsynaptic mapping of Drosophila mushroom body output neurons. Elife, 10, p.e63379. 
32. Talay, M., Richman, E.B., Snell, N.J., Hartmann, G.G., Fisher, J.D., Sorkaç, A., Santoyo, J.F., Chou-Freed, C., Nair, N., Johnson, M. and Szymanski, J.R., 2017. Transsynaptic mapping of second-order taste neurons in flies by trans-Tango. Neuron, 96(4), pp.783-795.

33. Jenett, A., Rubin, G.M., Ngo, T.-T. B., Shepherd, D., Murphy, C., Dionne, H., Pfeiffer, B.D., Cavallaro, A., Hall, D., Jeter, J., lyer, N., Fetter, D., Hausenfluck, J.H., Peng, H., Trautman, E., Svirskas, R., Myers, G.W., Iwinski, Z.R., Aso, Y., DePasquale, G.M., Enos, A., Hulamm, P., Lam, S.C.B., Li, H-H., Laverty, T., Long, F., Qu, L., Murphy, S.D., Rokicki, K., Safford, T., Shaw, K., Simpson, J.H., Sowell, A., Tae, S., Yu, Y., Zugates, C.T. A GAL4-Driver Line Resource for Drosophila Neurobiology. Cell Reports (2012) Doi: 10.1016/j.celrep.2012.09.011

34. Tirian, L., \& Dickson, B. J. The VT GAL4, LexA, and split-GAL4 driver line collections for targeted expression in the Drosophila nervous system. bioRxiv. 2017: 198648. DOI: 10.1101/198648

35. Pfeiffer, B. D., Ngo, T. B., Hibbard, K. L., Murphy, C., Jenett, A., Truman, J. W., \& Rubin, G. M. Refinement of Tools for Targeted Gene Expression in Drosophila. Genetics. 2010; 186: 735-755. DOI: $10.1534 /$ genetics.110.119917

36. Clyne, J.D., \& Miesenbock, G. (2008). Sex-specific control and tuning of the pattern generator for courtship song in Drosophila. Cell, 133, 354-363

37. Ren, Q., Awasaki, T., Huang, Y.F., Liu, Z. and Lee, T., 2016. Cell class-lineage analysis reveals sexually dimorphic lineage compositions in the Drosophila brain. Current Biology, 26(19), pp.25832593.

38. Donlea, J.M., Pimentel, D. and Miesenböck, G., 2014. Neuronal machinery of sleep homeostasis in Drosophila. Neuron, 81(4), pp.860-872.

39. Rayshubskiy, A., Holtz, S.L., D’Alessandro, I. , Li, A.A., Vanderbeck, Q.X., Haber, I.S., Gibb, P.W., and Wilson, R.I 2020. Neural circuit mechanisms for steering control in walking Drosophila bioRxiv 2020.04.04.024703; doi: https://doi.org/10.1101/2020.04.04.024703

40. Stone, T., Webb, B., Adden, A., Weddig, N.B., Honkanen, A., Templin, R., Wcislo, W., Scimeca, L., Warrant, E. and Heinze, S., 2017. An anatomically constrained model for path integration in the bee brain. Current Biology, 27(20), pp.3069-3085.

41. Samejima, K., Ueda, Y., Doya, K. and Kimura, M., 2005. Representation of action-specific reward values in the striatum. Science, 310(5752), pp.1337-1340.

42. Lau, B. and Glimcher, P.W., 2008. Value representations in the primate striatum during matching behavior. Neuron, 58(3), pp.451-463.

43. Schultz, W., Dayan, P. and Montague, P.R., 1997. A neural substrate of prediction and reward. Science, 275(5306), pp.1593-1599.

44. O'Keefe, J. and Dostrovsky, J., 1971. The hippocampus as a spatial map: Preliminary evidence from unit activity in the freely-moving rat. Brain research.

45. Hafting, T., Fyhn, M., Molden, S., Moser, M.B. and Moser, E.I., 2005. Microstructure of a spatial map in the entorhinal cortex. Nature, 436(7052), pp.801-806.

46. Owald, D. and Waddell, S., 2015. Olfactory learning skews mushroom body output pathways to steer behavioral choice in Drosophila. Current opinion in neurobiology, 35, pp.178-184.

47. Vogt, K., Schnaitmann, C., Dylla, K.V., Knapek, S., Aso, Y., Rubin, G.M. and Tanimoto, H., 2014. Shared mushroom body circuits underlie visual and olfactory memories in Drosophila. Elife, 3, p.e02395. 
48. Seelig, J.D. and Jayaraman, V., 2015. Neural dynamics for landmark orientation and angular path integration. Nature, 521(7551), pp.186-191.

49. Handler, A., Graham, T.G., Cohn, R., Morantte, I., Siliciano, A.F., Zeng, J., Li, Y. and Ruta, V., 2019. Distinct dopamine receptor pathways underlie the temporal sensitivity of associative learning. Cell, 178(1), pp.60-75.

50. Bell, W.J. and Kramer, E., 1979. Search and anemotactic orientation of cockroaches. Journal of Insect Physiology, 25(8), pp.631-640.

51. Yorozu, S., Wong, A., Fischer, B.J., Dankert, H., Kernan, M.J., Kamikouchi, A., Ito, K. and Anderson, D.J., 2009. Distinct sensory representations of wind and near-field sound in the Drosophila brain. Nature, 458(7235), pp.201-205.

52. Okubo, T.S., Patella, P., D'Alessandro, I. and Wilson, R.I., 2020. A neural network for wind-guided compass navigation. Neuron, 107(5), pp.924-940.

53. Kennedy, J.S., 1940. The visual responses of flying mosquitoes. In Proceedings of the Zoological Society of London (Vol. 109, No. pt. 4). London.

54. van Breugel, F. and Dickinson, M.H., 2014. Plume-tracking behavior of flying Drosophila emerges from a set of distinct sensory-motor reflexes. Current Biology, 24(3), pp.274-286.

55. Buehlmann, C., Hansson, B.S. and Knaden, M., 2012. Path integration controls nest-plume following in desert ants. Current Biology, 22(7), pp.645-649.

\section{Methods References:}

56. Giovannucci A, Friedrich J, Gunn P, Kalfon J, Brown BL, Koay SA, Taxidis J, Najafi F, Gauthier JL, Zhou P, Khakh BS, Tank DW, Chklovskii DB, Pnevmatikakis EA. 2019. CalmAn an open source tool for scalable calcium imaging data analysis. eLife 8:e38173. DOI: https://doi.org/10.7554/eLife.38173, PMID: 30652683

57. Pnevmatikakis EA, Giovannucci A. 2017. NoRMCorre: an online algorithm for piecewise rigid motion correction of calcium imaging data. Journal of Neuroscience Methods 291:83-94. DOI: https://doi.org/10.1016/j.jneumeth.2017.07.031, PMID: 28782629

58. Muir DR, Kampa BM. 2014. FocusStack and StimServer: a new open source MATLAB toolchain for visual stimulation and analysis of two-photon calcium neuronal imaging data. Frontiers in Neuroinformatics 8:85. DOI: https://doi.org/10.3389/fninf.2014.00085, PMID: 25653614

59. Nagel KI, Wilson RI. 2016. Mechanisms underlying population response dynamics in inhibitory interneurons of the Drosophila Antennal Lobe. The Journal of Neuroscience 36:4325-4338. DOI: https://doi.org/10.1523/JNEUROSCI.3887-15.2016, PMID: 27076428 
Figure 1

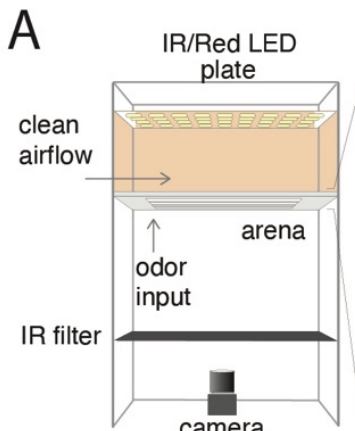

C
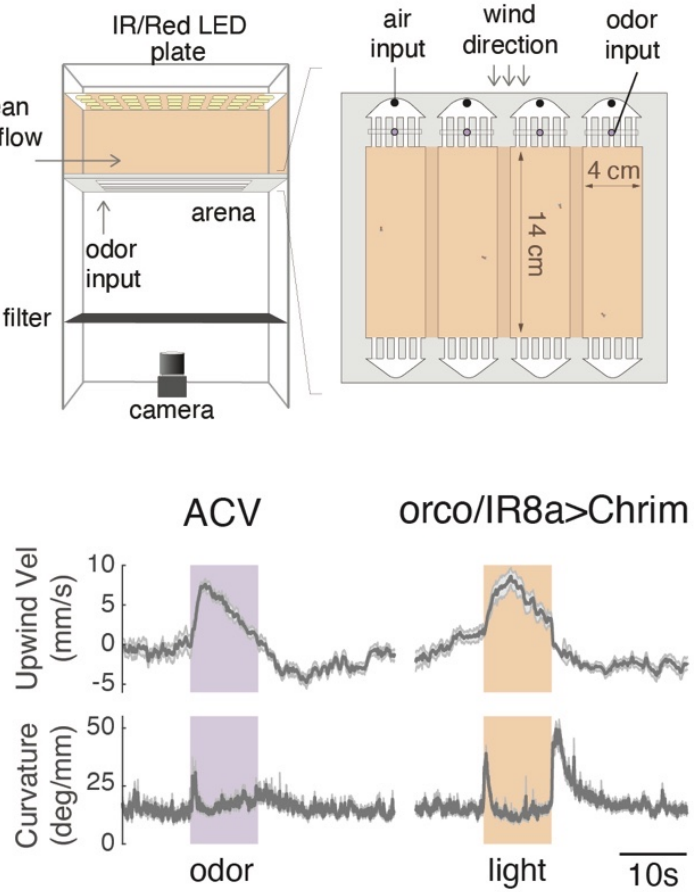

orco/IR8a>Chrim
B

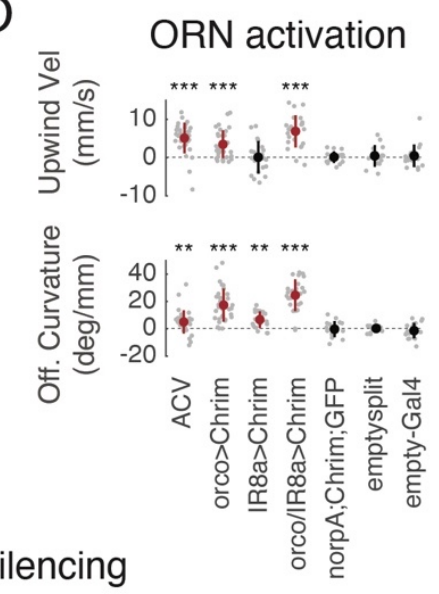

D

orco/IR8a>Chrim
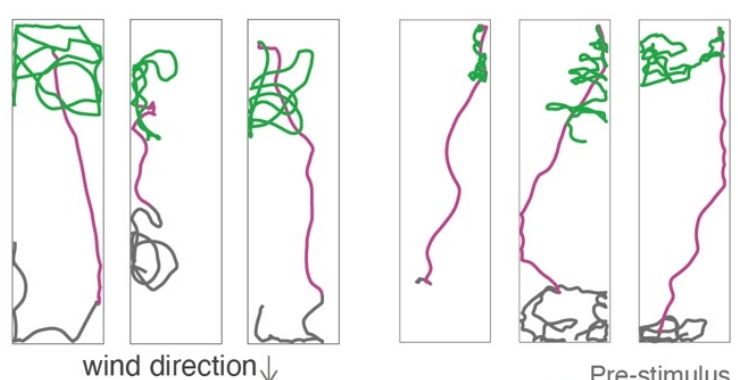

Pre-stimulus Odor/Light (10s) Post-stimulus
$\mathrm{E}$

TNT/ orco/IR8a>TNT
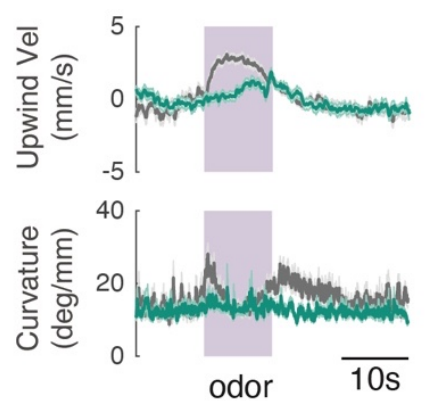

$\mathrm{F}$

ORN silencing

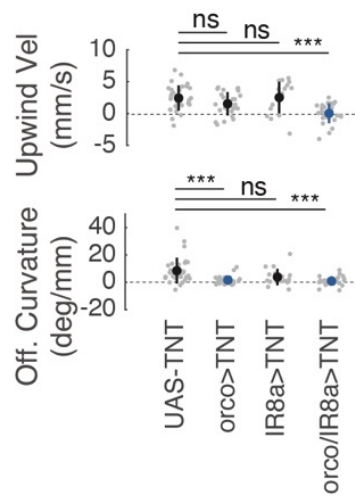

Fig. 1: ORN stimulation is necessary and sufficient for olfactory navigation motor programs A) Schematic of top and side view of the behavioral apparatus showing IR illumination (850nm), red activation light $\left(626 \mathrm{~nm}, 26 \mu \mathrm{W} / \mathrm{mm}^{2}\right)$, imaging camera, behavioral chambers, and air and odor inputs. B) Example walking trajectories in response to ORN stimulation with apple cider vinegar (left, ACV, 1\%) and optogenetic activation of orco+ and IR8a+ ORNs (right), before (gray), during (magenta), and after (green) 10s of odor (left) or light (right). Wind was constant at $11.9 \mathrm{~cm} / \mathrm{s}$.

C) Time course of upwind velocity and curvature (angular/forward velocity) in response to stimulation, averaged across flies (mean \pm SEM, ACV N=26 flies, ORN activation $\mathrm{N}=24$ flies). shaded area:

stimulation period (10s $1 \% \mathrm{ACV}$ (purple) or 10s light (orange) respectively). 
D) Upwind velocity and offset curvature (average change from baseline for single flies) in response to stimulation for each genotype/condition. Mean \pm STD overlaid; red indicates a significant increase. ACV, orco $>$ Chrimson, and orco/IR8a $>$ Chrimson stimulation all drove significant increases in upwind velocity (0-5 s after stimulus onset, Wilcoxon signed rank test: $p=1.0997 e-05,3.8672 e-05,2.6691 e-05)$ and offset curvature (0-2 s after stimulus offset, Wilcoxon signed rank test $p=1.9619 \mathrm{e}-04,1.1742 \mathrm{e}-06$, 2.3518e-05). Light activation of flies carrying only the parental effector (norpA;UAS-Chrimson), emptyGAL4>Chrimson, or empty-split GAL4>Chrimson did not increase upwind velocity (Wilcoxon signed rank test: $p=0.7174,0.6874,0.6698$ ) or offset curvature (Wilcoxon signed rank test: $p=0.7960,0.3144$ ,0.7354). IR8a $>$ Chrimson stimulation did not increase upwind velocity $(p=0.3507)$ but did increase offset curvature $(p=4.4934 \mathrm{e}-04)$.

E) Time course of upwind velocity and curvature in response to odor in flies with all ACV-sensitive ORNs silenced (orco/IR8a>TNT, mean \pm SEM, $N=26$, teal) versus control (UAS-TNT, $N=31$, gray). F) Upwind velocity and offset curvature for silencing experiments, quantified as in D. Blue overlay represents significant decrease compared to control (UAS-TNT). (Mann-Whitney U test compared to UAS-TNT control, upwind velocity: $p=0.10627,0.40095,0.00010917$, offset curvature: $3.2758 \mathrm{e}-05$, $0.037135,4.2482 \mathrm{e}-05)$

All comparisons were corrected using the Bonferroni method (see Materials and Methods). 
Figure S1
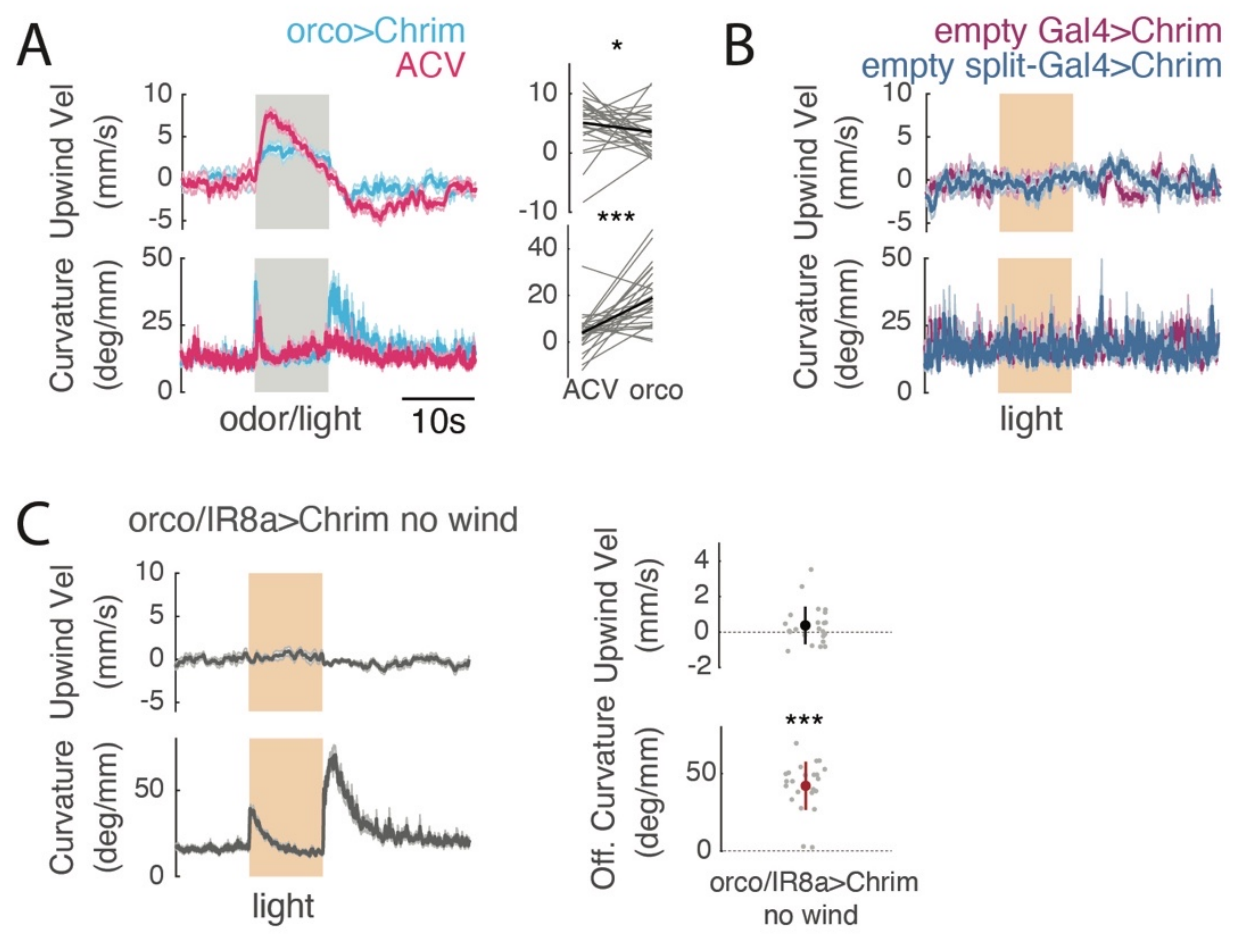

D

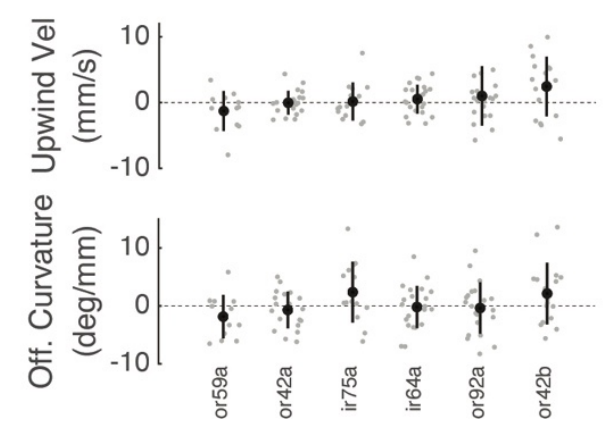

E orco silencing
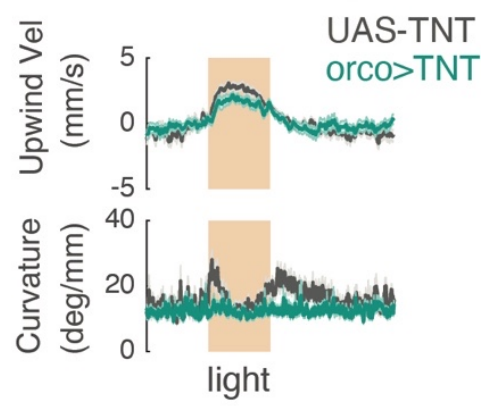

Fig. S1: Peripheral encoding of olfactory navigation motor programs

A) Left: Upwind velocity and curvature time courses (mean $\pm \mathrm{SEM}$ ) in the same flies ( $\mathrm{N}=26$ flies) exposed to both $1 \%$ ACV (pink) and optogenetic stimulation of orco+ ORNs (blue) in interleaved trials. Right: Comparison of average upwind velocity ( $0-5 \mathrm{~s}$ after stimulus onset) and offset curvature (0-2s after stimulus offset) for each fly. Within each fly, ACV evoked greater upwind velocity than orco stimulation (Wilcoxon signed rank test: $\mathrm{p}=0.0319$ ) but weaker offset curvature than orco stimulation (Wilcoxon sign rank test: $\mathrm{p}=1.3216 \mathrm{e}-04$ ).

B) Upwind velocity and curvature time courses (mean \pm SEM) for control flies empty-GAL4>Chrimson (purple, $\mathrm{N}=19$ ) and empty split-GAL4>Chrimson (blue, $\mathrm{N}=14$ ). No significant change in either parameter during stimulation (quantified in Figure 1D). 
C) Right: Upwind velocity and curvature time courses (mean \pm SEM) for orco/IR8a>Chrimson flies in the absence of wind ( $\mathrm{N}=24$ flies, see also $\{8,28\})$. Left: quantification as in Fig. 1D. No increase in upwind velocity (Wilcoxon signed rank test, $p=0.0191$ ), but offset curvature increases significantly increases after stimulation (Wilcoxon signed rank test, $\mathrm{p}=1.8215 \mathrm{e}-05$ ).

D) Upwind velocity and offset curvature (average change from baseline for single flies, mean \pm STD overlaid) for each single ORN that responds to ACV (25). No significant increase in upwind velocity (Wilcoxon sign rank test, $p=0.2334,0.7089,0.9032,0.3304,0.3754,0.0442$ ) or offset curvature (Wilcoxon sign rank test, $\mathrm{p}=0.1763,0.3317,0.0785,0.7151,0.6639,0.1488$ ).

E) Timecourse of upwind velocity and curvature for flies with orco>TNT ( $N=25$ flies, teal) versus UASTNT controls ( $\mathrm{N}=31$ flies, gray).

All comparisons for behavioral experiments Bonferroni corrected for multiple comparisons. 
Figure 2

A

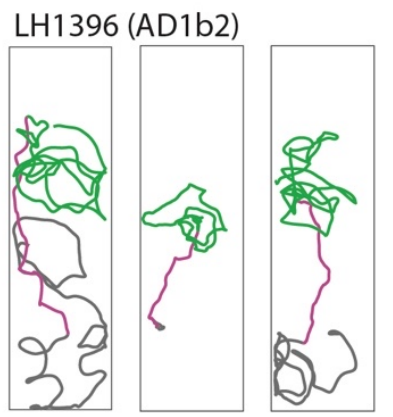

wind direction $\downarrow$

C

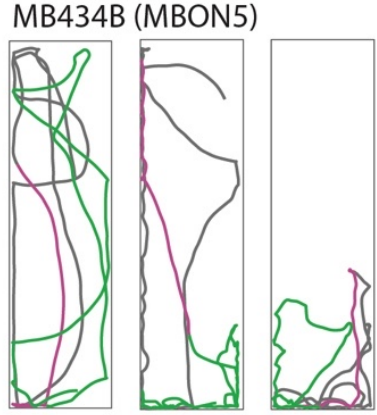

B

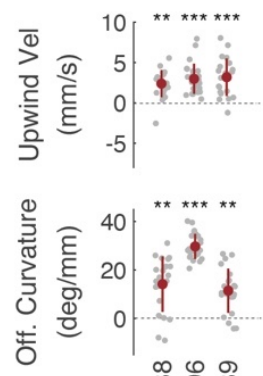

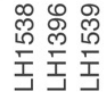

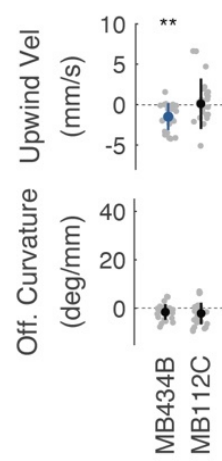

MB052B (MBON15-19)

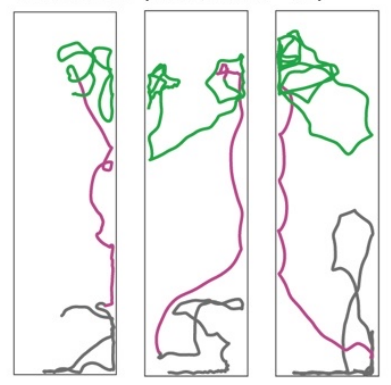

$\mathrm{D}$

MB112C (MBON11)

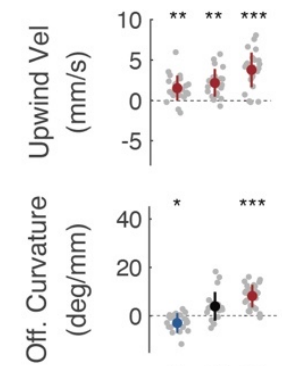

요 $\stackrel{m}{\sim}$

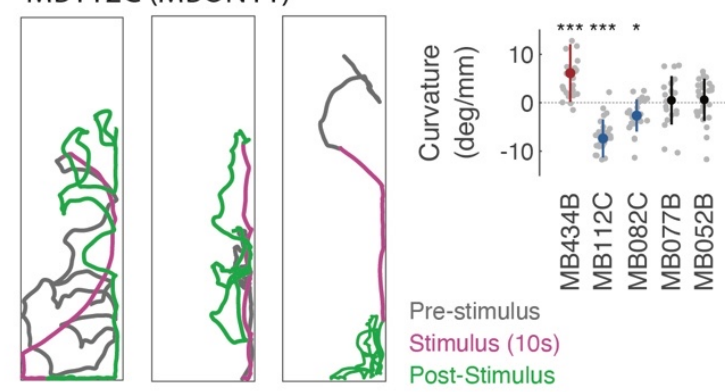

$E$
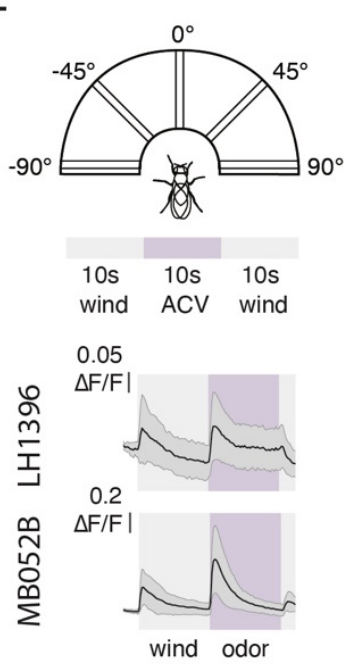
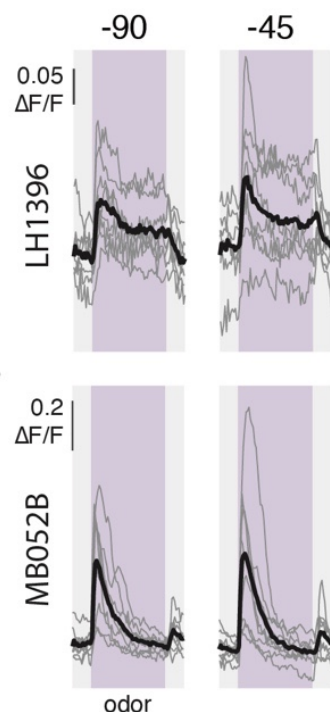
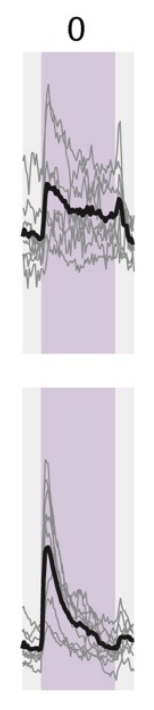
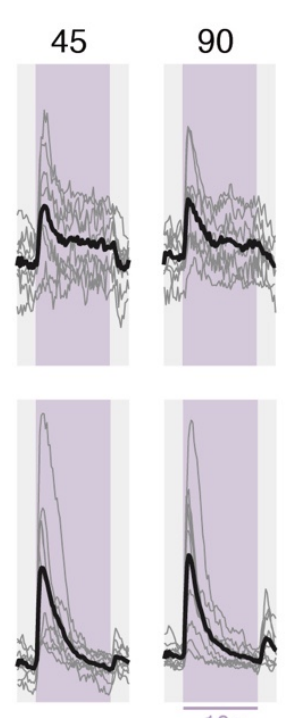

$\mathrm{F}$
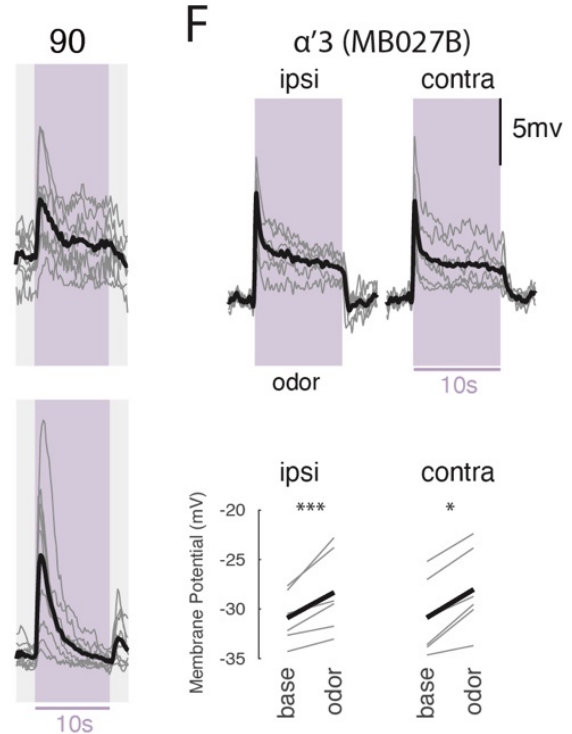

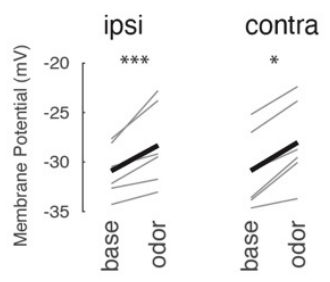

Fig. 2: Specific LH and MB output neurons promote motor programs for olfactory navigation and respond to odor independent of wind direction

A) Example behavioral trajectories in response to optogenetic activation of AD1b2 LHONs labelled by the line LH1396 (left). Right: Upwind velocity and offset curvature (quantified as in Fig. 1D) for three lines that label AD1b2 LHONs (LH1538, LH1539, LH1396). All three lines significantly increase both upwind velocity and offset curvature (Wilcoxon signed rank test, upwind: $p=2.4548 e-04$, 7.9941e-05, 1.8215e-05; offset-curvature: $p=3.6712 \mathrm{e}-04,1.7699 \mathrm{e}-04,1.8215 \mathrm{e}-05$ respectively) 
B) Example behavioral trajectories in response to optogenetic activation of MBONs15-19 labelled by the line MB052B (left). Right: Upwind velocity and offset curvature quantification for three cholinergic MB lines: MB052B, MB077B, and MB082C. Each line labels distinct MBONs. All increase upwind velocity (Wilcoxon signed rank test, $p=1.0997 \mathrm{e}-05,1.2267 \mathrm{e}-04,2.0378 \mathrm{e}-04$ ) while MB052B increases offset curvature $(p=6.2811 \mathrm{e}-06)$, MB077B does not $(p=0.0046)$ and MB082C reduced offset curvature $(p=0.0018)$.

C) Example behavioral trajectories in response to optogenetic activation of glutamatergic MBONs 5 and 6, labeled by the line MB434B (left). Right: Upwind velocity and offset curvature for MB434B and MB112C. MB434B significantly decreases upwind velocity (Wilcoxon signed rank test $p=2.2806 e-04$ ) while MB112C does not affect upwind velocity (Wilcoxon signed rank test, $p=0.8036$ ). Neither affects offset curvature $(p=0.0258,0.0179)$.

D) Example behavioral trajectories in response to optogenetic activation of the GABAergic MBON11, labeled by the line MB112C (left). Right: Curvature during stimulus (from 2-5s after stimulus onset) for MB112C, MB434B, MB052B, and MB077B. MB112C significantly reduces curvature during the stimulus (Wilcoxon signed rank test, $p=3.5150 e-06)$ while MB434B increases curvature $(p=4.3941 e-$ 05) and other MB genotypes have no effect $(p=0.4979,0.2297)$.

E) Calcium responses $(\Delta F / F)$ measured in the dendritic processes of the LH labelled by LH1396 (N=8 flies) and output processes labelled by MB052B ( $N=9$ flies) using GCaMP6f in response to odor (10\% ACV, purple) and wind (gray) delivered from 5 directions (schematic). Gray traces represent individual flies, black traces represents mean across flies. No significant differences in response as a function of direction were observed for either LH1396 or MB052B (ANOVA: $F(4,35)=0.35, p=0.8408, F(4,40)=0.3$, $\mathrm{p}=0.8794$ ). Lower left inset: Average odor and wind responses for $\mathrm{LH} 1396$ and MB052B (averaged across directions, mean \pm STD). In LH1396, odor elicits a more sustained response than wind alone. In MB052B odor elicits a larger response that wind alone.

F) Membrane potential responses in a'3 MBONs (labeled by MB027B) to $10 \%$ ACV presented from $90^{\circ}$ ipsilateral or contralateral to the recorded neuron. Black trace represents mean across flies while gray traces are individual flies ( $\mathrm{N}=6$ cells per hemisphere, each from $1 \mathrm{fly}$ ). Odor significantly increases membrane voltage both ipsilaterally and contralaterally (paired student t-test $p=0.0018,0.0159$ ) and is not different between sides (unpaired student t-test $p=0.7561$ ).

All comparisons for behavioral data were corrected using the Bonferroni method (see Materials and Methods). 
Figure S2

A activation timecourses
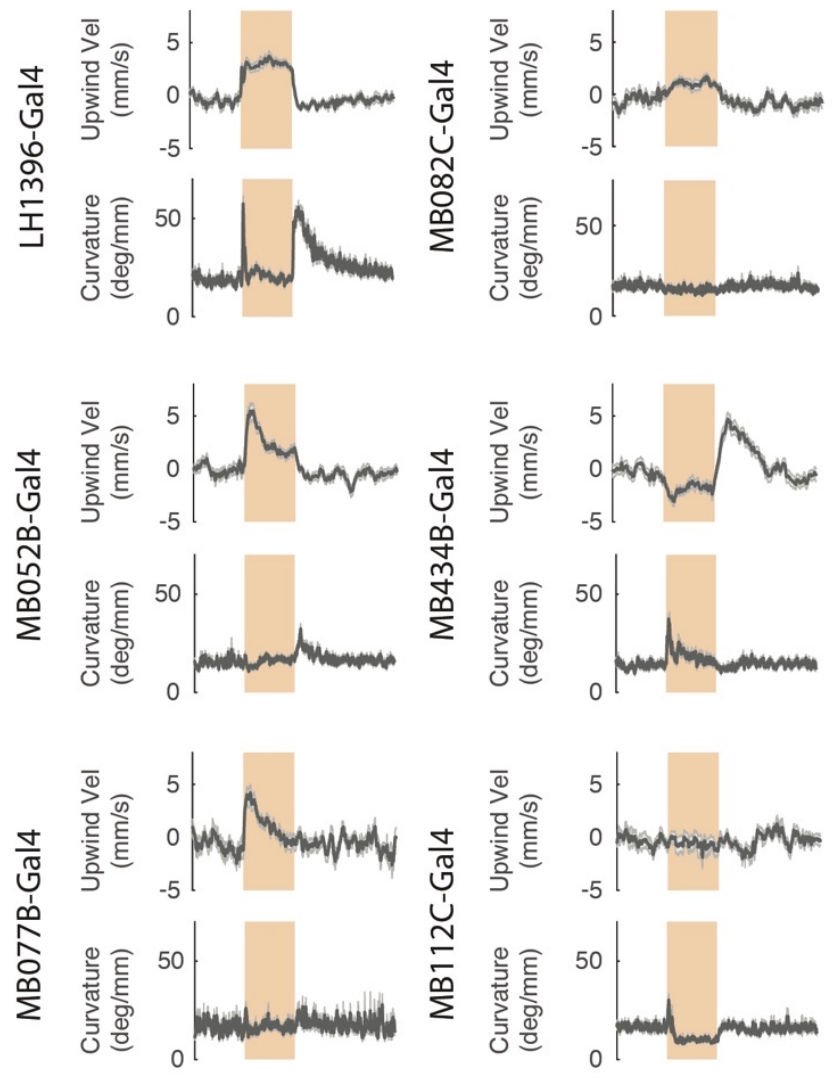

D

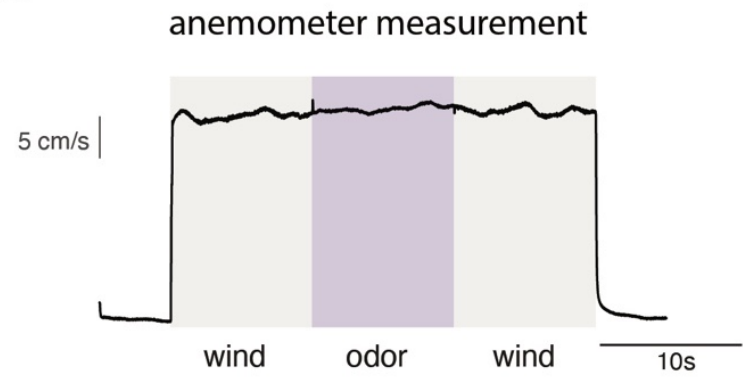

$\mathrm{E}$

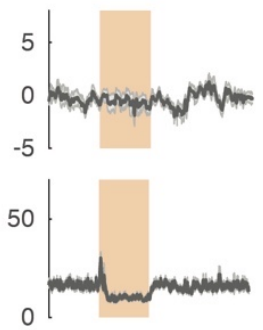

B

additional MBON/LHON activation

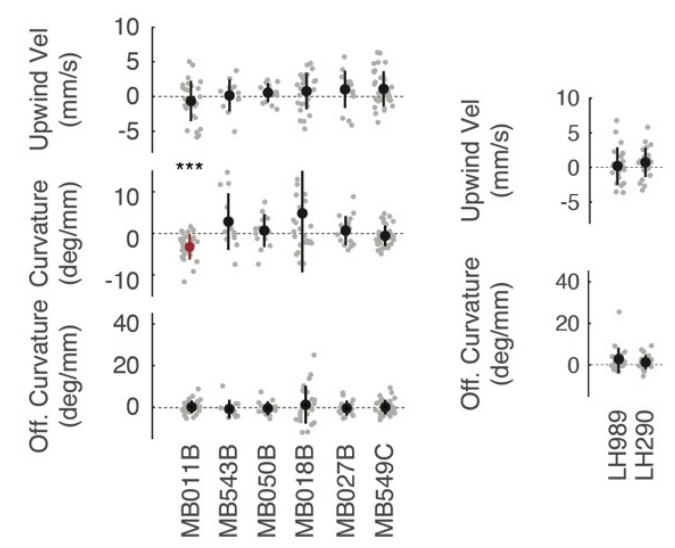

C

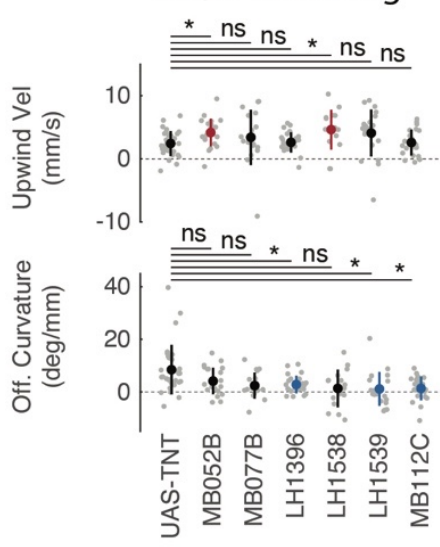

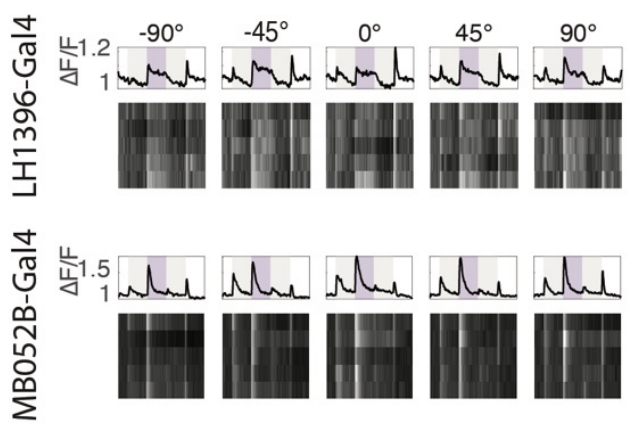

Fig. S2: Additional data on LH and MB responses and behavior

A) Upwind velocity and curvature time courses (mean \pm SEM) for LH1396>Chrimson ( $\mathrm{N}=24$ flies), MB052B $>$ Chrimson ( $N=27)$, MB077B $>$ Chrimson $(\mathrm{N}=21)$, MB082C >Chrimson $(\mathrm{N}=24)$,

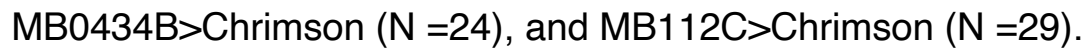

B) Upwind velocity, curvature during stimulus, and offset curvature for individual MBONs labeled by MB052B (left) and for additional LHONs (right), mean \pm STD overlaid. MBONs: upwind: 0.3275, 0.5693, 0.1726, 0.1363, 0.1477, 0.0360, curvature: 3.4071e-05, 0.3804, 0.5016, 0.2029, 0.5695, 0.0742 offset 
curvature: $0.8376,0.2061,0.3910,0.7533,0.4380,0.7807$. LHONs: upwind: $0.9095,0.0975$, offset curvature: $0.1997,0.1443$.

C) Upwind velocity and offset curvature (quantified as in Fig. 1D) when LHONs and MBONs are silenced. (Mann Whitney U test compared to UAS-TNT control, upwind velocity: $0.0036862,0.17444$, $0.6529,0.0036449,0.0090304,0.83555)$ offset curvature: $0.063702,0.012398,0.0027408,0.013958$, 0.00070953, 0.0011212)

D) Average stimulus delivered to the fly from 3 trials from each of the 5 directions as measured by a hotwire anemometer. There is no change in windspeed between wind alone and wind + odor.

E) Single fly examples of calcium responses to wind and odor in LH1396 (top) and MB052B (bottom). Top boxes show average traces for single flies across 5 trials from each direction. Heat maps below depict responses for individual trials 
Figure 3

A
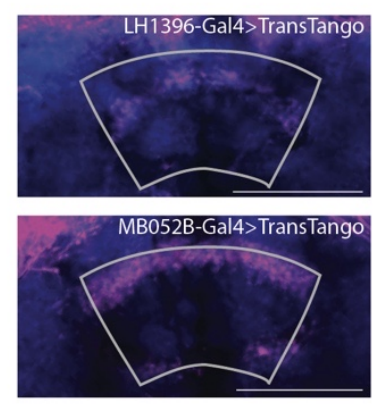

B

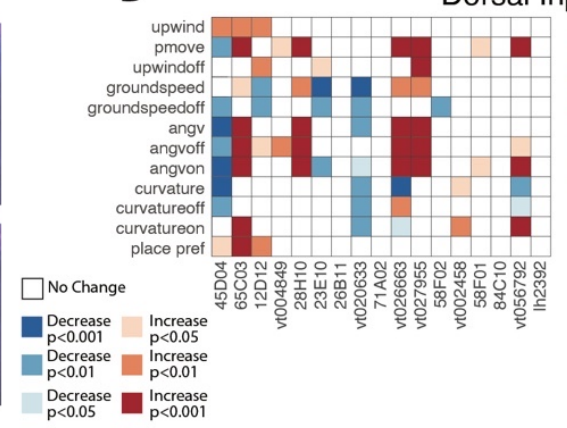

Dorsal Inputs

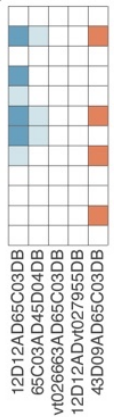

Ventral Inputs

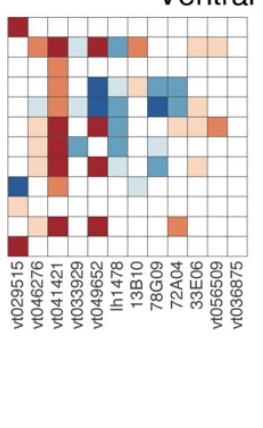

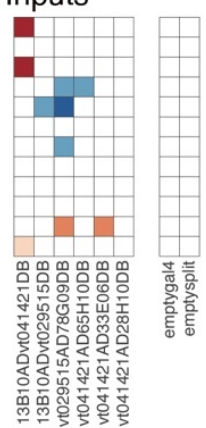

C
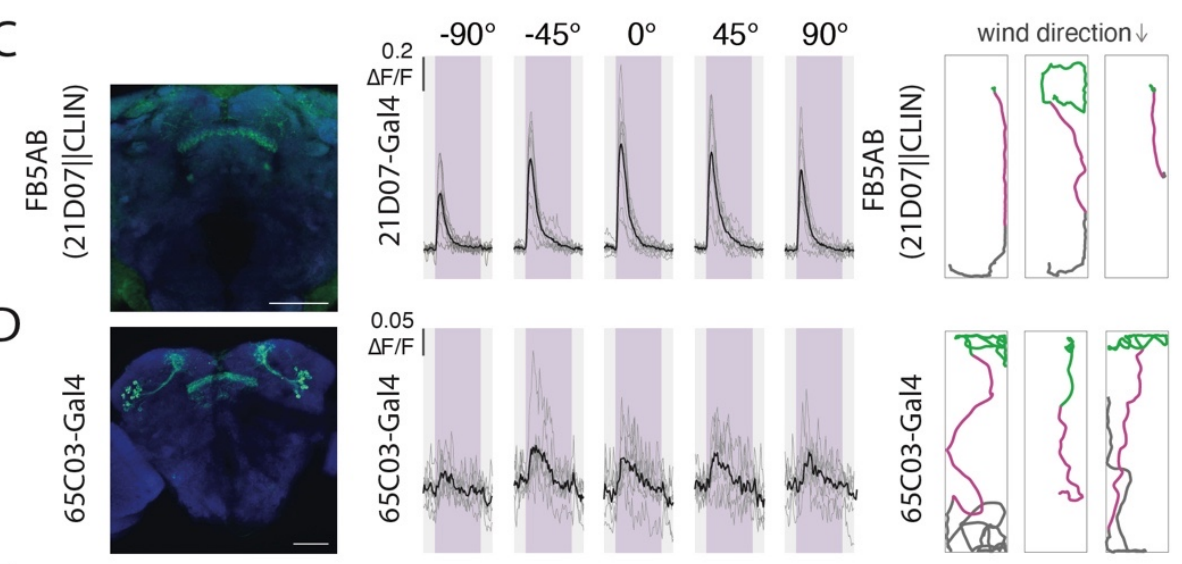

Upwind Velocity $(\mathrm{mm} / \mathrm{s})$

Offset Curvature
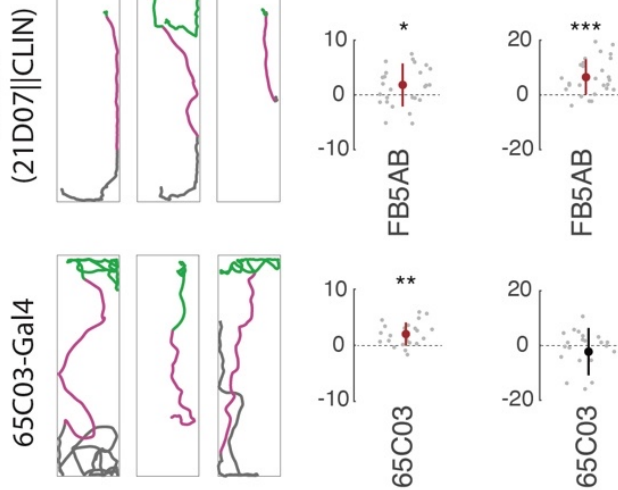

E
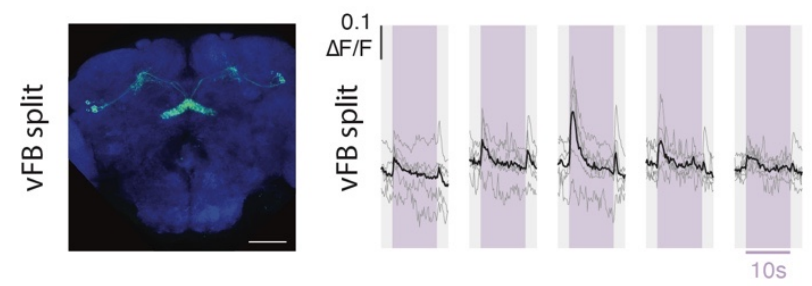
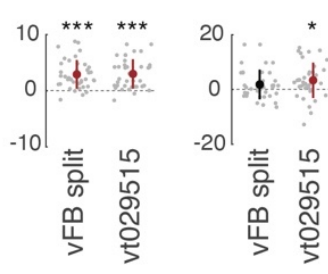

\section{Fig. 3: Tangential FB inputs that respond to odor and drive upwind orientation}

A) Trans-tango signal driven by LH1396-GAL4 (top) and MB052B-GAL4 (bottom). Trans-synaptic signal (magenta) was observed in the dorsal FB in both cases. Neuropil is shown in blue. The FB is outlined in gray. Scale bar 50uM.

B) Summary of optogenetic activation screen of dorsal (left) and ventral (center) tangential FB inputs, and negative controls (right). See Materials and Methods for calculation of behavioral parameters and supplemental table for exact magnitudes.

C) Left: The neuron FB5AB is labeled by 21D07-GAL4; filtering this driver through CLIN limits expression to type II neuroblasts which comprise the central complex. Confocal image of mVenus expressed with UAS-Chrimson under 21D07IICLIN. Center: Calcium response in 21D07-GAL4 to the same stimulus shown in Fig. 2E. 10s odor period in purple. Gray traces represent individual flies and black traces represent the average across flies $(\mathrm{N}=9)$. No significant difference was observed between directions (ANOVA: $F(4,40)=2.14, p=0.0938$ ). Right: Example behavioral trajectories and quantification 
of upwind velocity and offset curvature in 21D07IICLIN>Chrimson flies. Light intensity for this experiment only was $34 \mu \mathrm{W} / \mathrm{mm}^{2}$. For this figure upwind velocity was quantified over $0-10$ s after odor onset as upwind responses were slower and more long lasting for FB drivers (Supp. Fig. 4F). Mean \pm STD overlaid (Wilcoxon signed rank test: $p=0.0306,8.1448 \mathrm{e}-05$ ).

D) Left: The line 65C03-GAL4 labels a set of dorsal FB tangential inputs and some ventral FB inputs. Confocal image of mVenus expressed with UAS-Chrimson by this driver. Center: Calcium responses of 65C03-GAL4 to 10s of odor. Gray traces represent individual flies and black traces represent averages across flies $(N=7)$. No significant different between directions (ANOVA $F(4,30)=0.68$, $\mathrm{p}=0.6096)$. Right: Example behavioral trajectories quantification of upwind velocity and offset curvature in 65C03-GAL4 flies. Mean \pm STD overlaid. (Wilcoxon signed rank test: $p=4.1850 e-04,0.5841$ ) E) Left: Ventral FB inputs labeled by the split GAL4 line 13B10AD;vt041421DB (vFB split). Confocal image of mVenus expressed with UAS-Chrimson by this driver. Center: Calcium responses of 13B10AD;VT041421DB in response to 10s of odor. Gray traces represent individual flies and black traces represent average across flies $(\mathrm{N}=6)$. No significant difference in response across directions (ANOVA: $F(4,25)=0.64, p=0.6358)$. Right: Example behavioral trajectories and quantification of upwind velocity and offset curvature in 13B10AD;vt041421DB and VT029515-GAL4, another line that labels ventral FB inputs, mean \pm STD overlaid (Wilcoxon signed rank test: VFB split $p=3.4153 e-07,0.1155$, VT029515-GAL4 p=4.3255e-07, 0.0024).

All comparisons for behavioral data were corrected using the Bonferroni method (see materials and methods) 


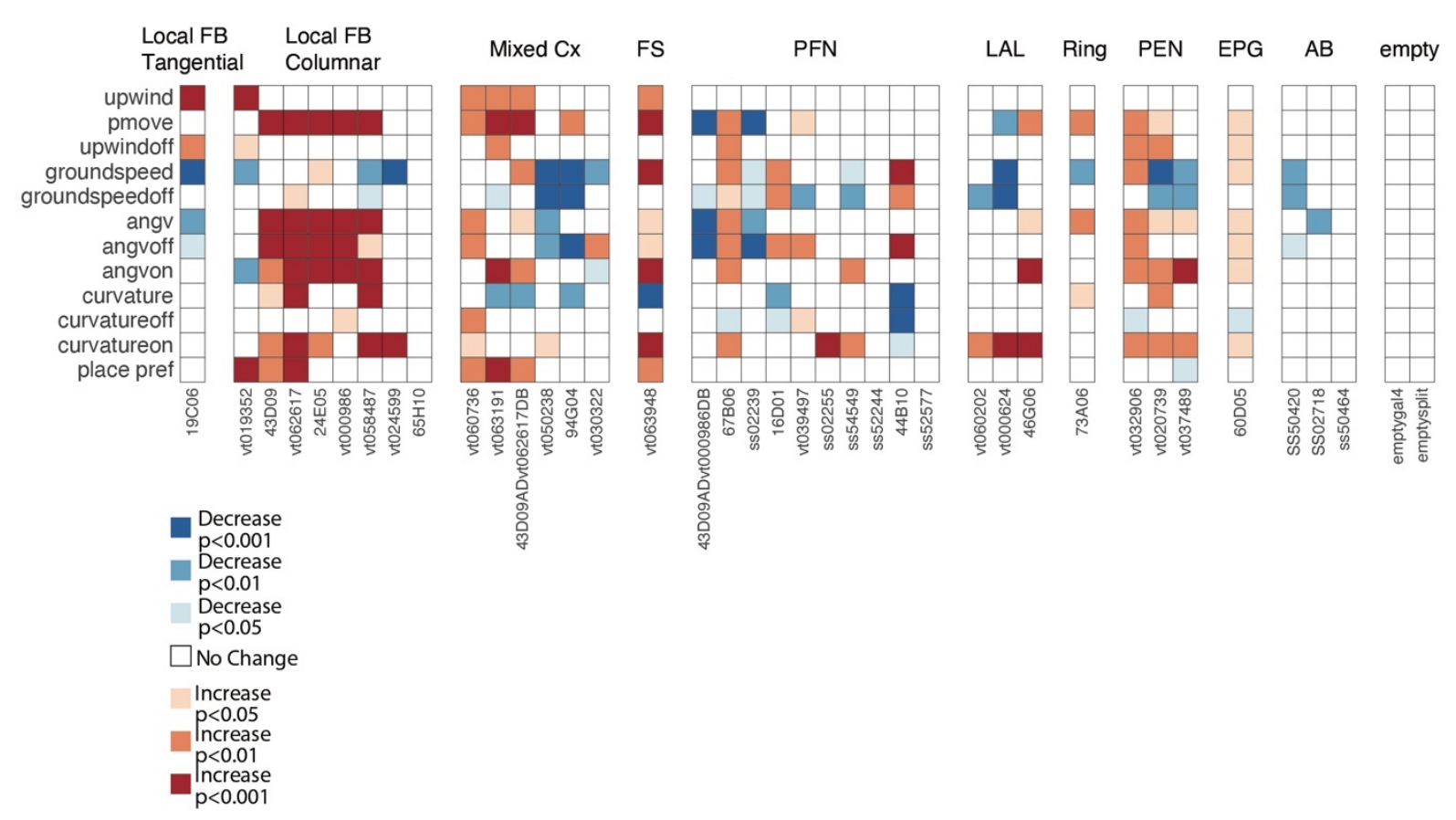

\section{Fig. S3: Additional Cx neuron optogenetic activation screen results}

A) Summary of optogenetic activation screen for additional central complex neuron types (Local FB neurons, PFNs, FS neurons, LAL innervating neurons, $A B$ neurons, PEN, ring and EPG neurons and drivers that labelled more than one $\mathrm{Cx}$ type prominently (mixed)). One local tangential FB neuron line (19C06-GAL4), one local columnar FB neuron line (VT019352-GAL4) and three mixed Cx lines (VT060736-GAL4, VT063191-GAL4, and the split line 43D09-AD; VT062617-DB) and one FS neuron line (VT063948-GAL4) produced significant increases in upwind velocity. No PFN, PEN, or AB lines produced significant changes in upwind velocity, nor did activation of EPG compass neurons (60D05GAL4). Several FB local columnar neuron lines drove increases in movement probability (Pmove) and angular velocity (angv): 43D09-GAL4, VT062617-GAL4, 24E05-GAL4, VT000986-GAL4, VT058487GAL4. See Materials and Methods for calculation of behavioral parameters. 
Figure 4
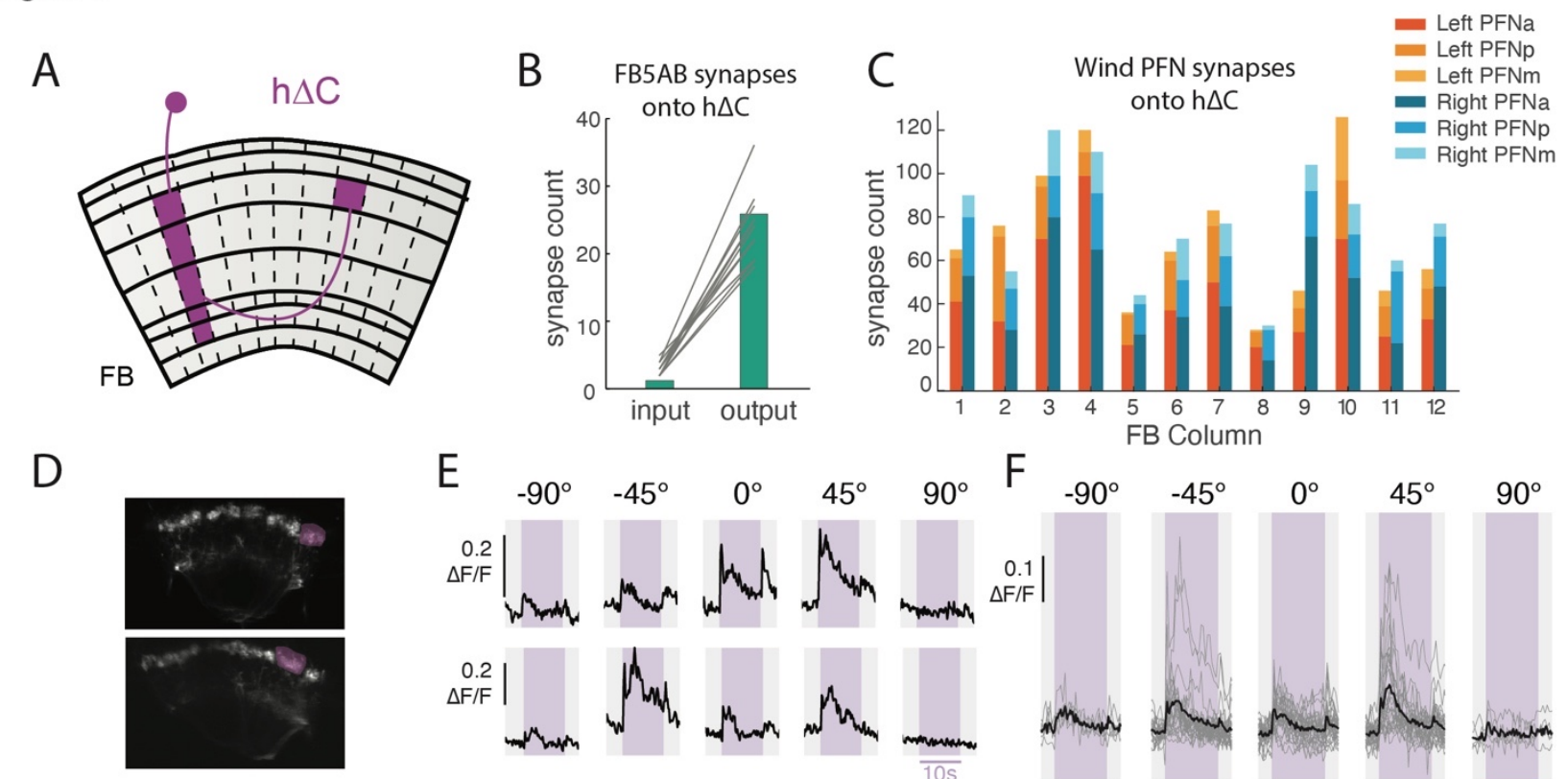

$\mathrm{E}$

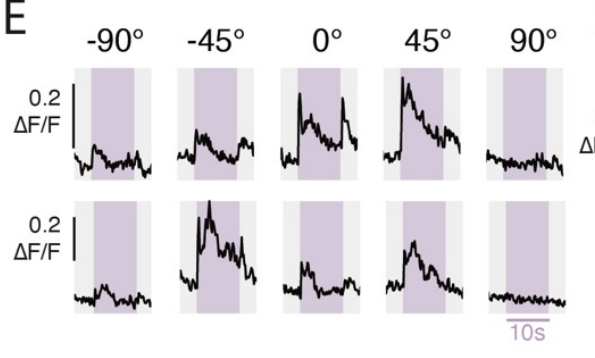

$\mathrm{F}$ FB Column
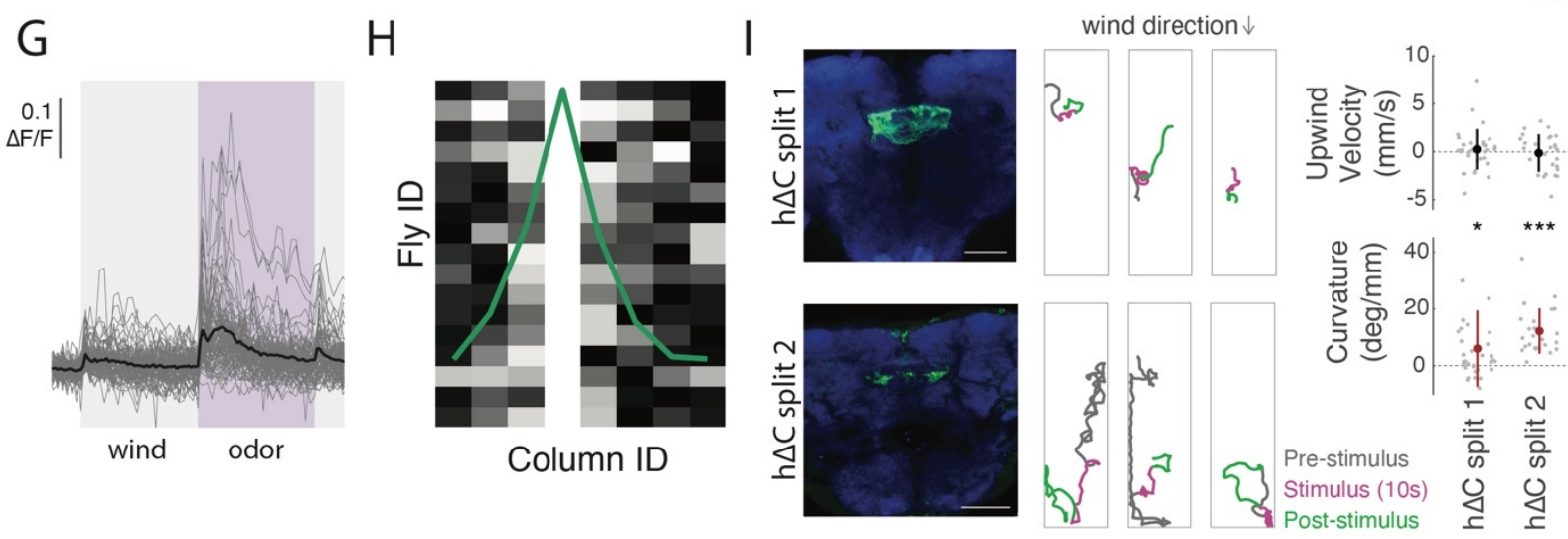

Fig. 4: $\mathrm{h} \Delta \mathrm{C}$ neurons integrate odor and wind direction information and drive turning

A) Cartoon schematic of the anatomy of an individual $\mathrm{h} \Delta \mathrm{C}$ neuron (purple). The $20 \mathrm{~h} \Delta \mathrm{C}$ neurons that tile the FB each receive input in layers 2-6 in a single column, and make output tufts exclusively in layer 6 , halfway across the FB from their inputs.

B) Number of FB5AB synapses onto the input and output tuft of $h \triangle C$ for every FB5AB-h $\triangle C$ pair.

C) Number of synapses from left and right windPFNs (PFNa, PFNp, and PFNm, tuned to $45^{\circ}$ left and right respectively) onto $\mathrm{h} \Delta \mathrm{C}$ neurons, summed within columns.

D) 2-photon image of tdTOM expressed with GCaMP6f under VT062617-GAL4 which labels $\mathrm{h} \triangle \mathrm{C}$ neurons. Purple ROI drawn around output tuft of a single column for analysis.

E) Odor responses of two example flies/columns showing directionally-tuned odor responses.

F) Summary of all measured odor responses $>$ 2STD above baseline across columns and flies. Gray traces represent individual columns and black traces represent mean across columns. 
G) Summary of wind and odor responses for all all measured responses > 2STD above baseline across columns and directions. Responses to odor are stronger than those to wind alone $(\mathrm{n}=87$ responsive columns from $\mathrm{N}=16$ Flies).

H) Directional responses are restricted to nearby columns: maximally responsive direction for each fly, where data is phase shifted so maximum columns align at column 4 . Each fly normalized to maximum column response.

I) Left: Confocal images of mVenus expressed with UAS-Chrimson driven by two h $\triangle \mathrm{C}$ split GAL4 lines: 19G02AD;VT062617DB (top) and VT026434AD;VT062617DB (bottom). Center: example behavioral trajectories driven by $\mathrm{h} \triangle \mathrm{C}$ split GAL4 activation. Right: Upwind velocity and curvature during stimulus (average change from baseline for single flies), mean \pm STD overlaid. $\mathrm{h} \Delta \mathrm{C}$ activation produces a significant increase in curvature (Wilcoxon signed rank test $p=0.0143,5.6061 \mathrm{e}-06$ ) but not upwind velocity (Wilcoxon signed rank test, $\mathrm{p}=0.3595,0.8288$ ). Scale bar represents $50 \mu \mathrm{M}$. 
bioRxiv preprint doi: https://doi.org/10.1101/2021.04.21.440842; this version posted April 22, 2021. The copyright holder for this preprint (which was not certified by peer review) is the author/funder, who has granted bioRxiv a license to display the preprint in perpetuity. It is made available under aCC-BY-NC-ND 4.0 International license.

Figure $\mathrm{S} 4$
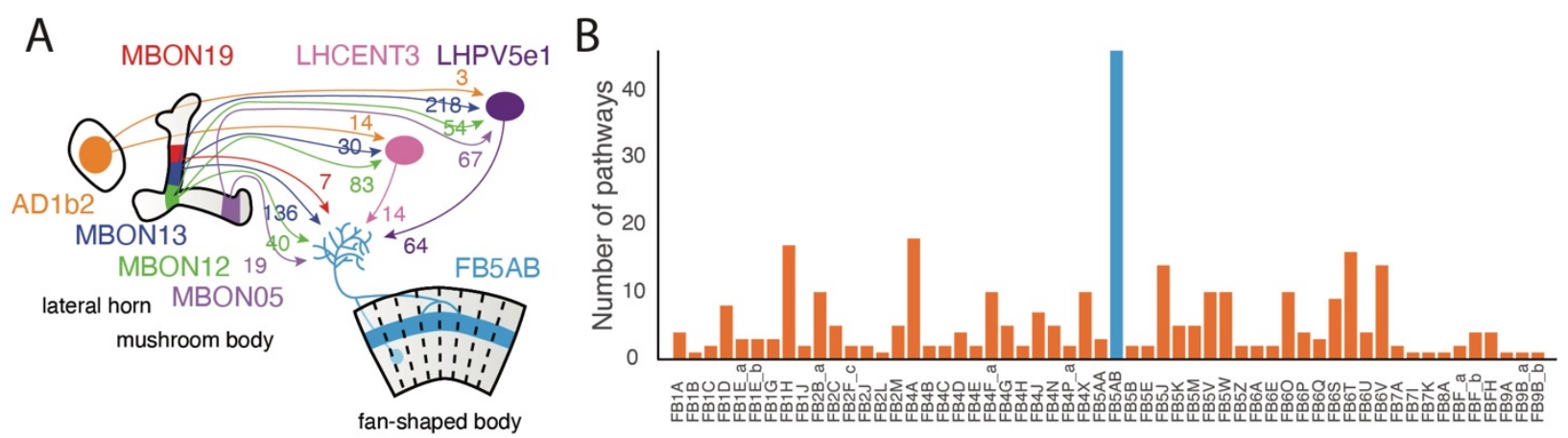

C
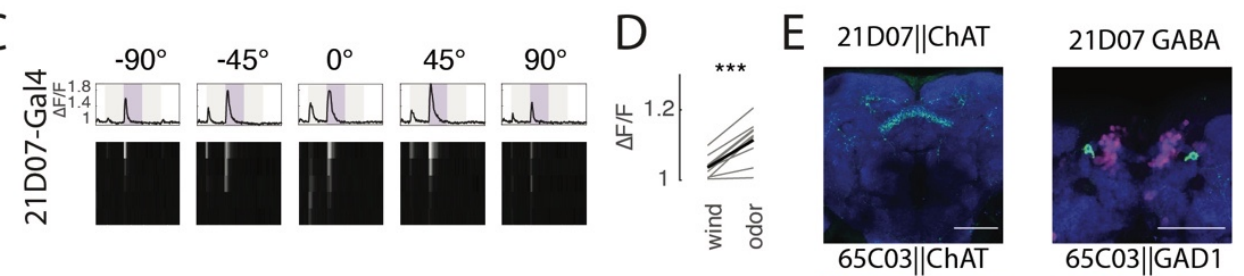

\section{$\mathrm{F}$}
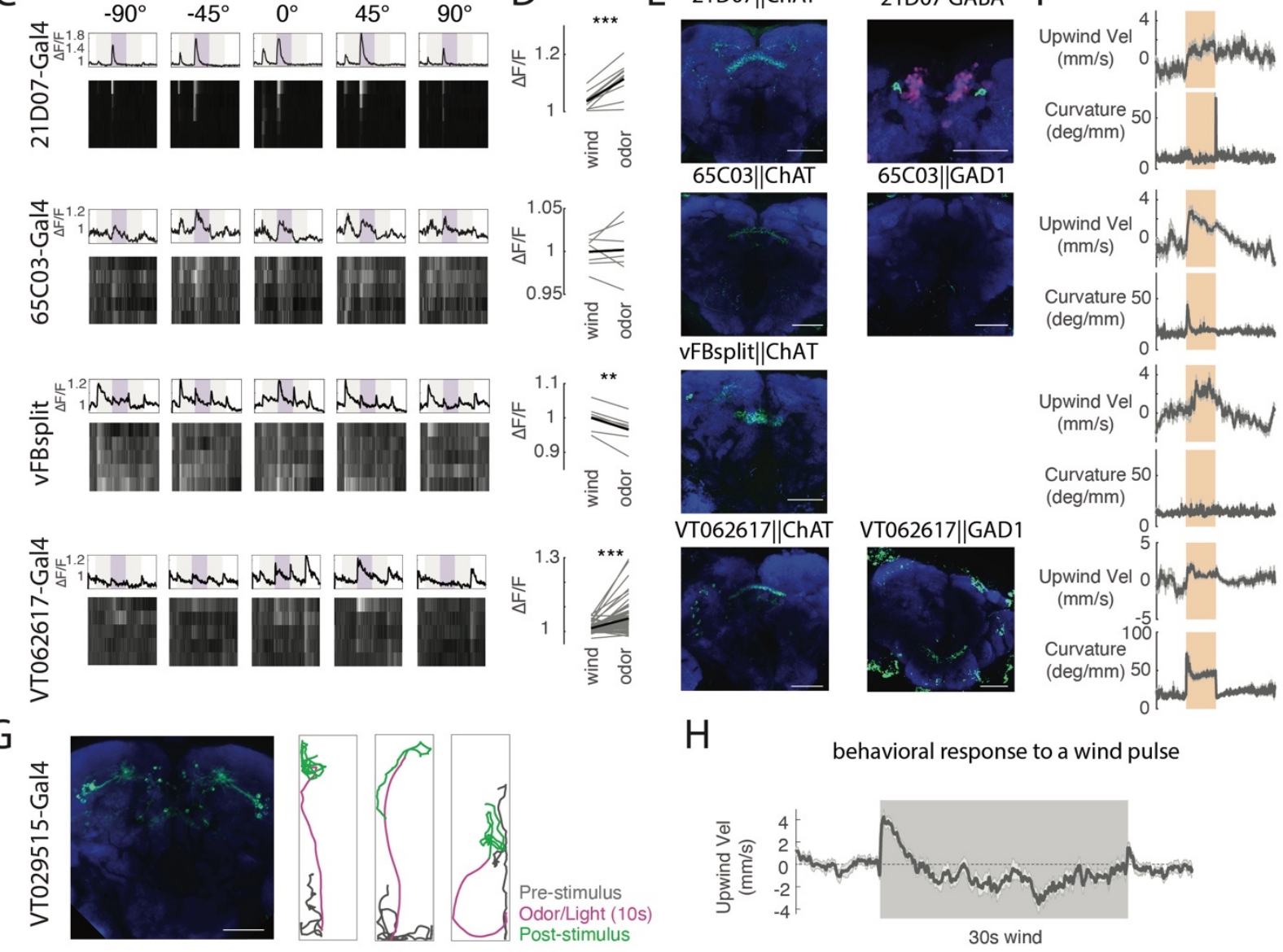

$\mathrm{H}$

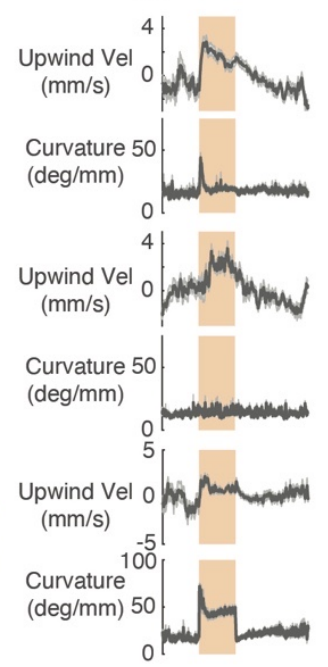

behavioral response to a wind pulse

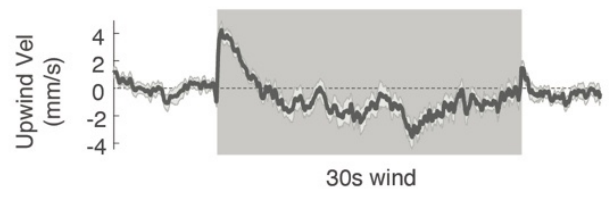

FB neuron silencing

$J$ $\mathrm{h} \Delta \mathrm{C}$ split 1 activation

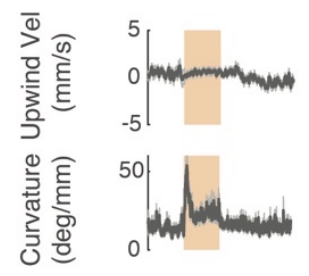

$\mathrm{h} \Delta \mathrm{C}$ split 2 activation

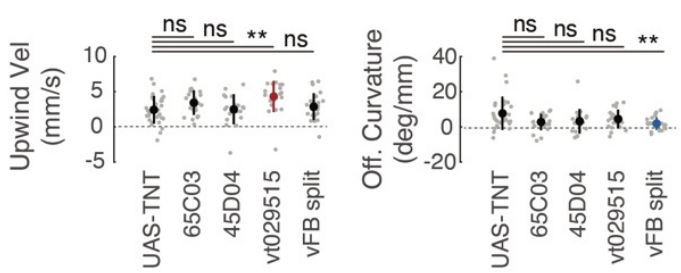

VT062617||GAD1
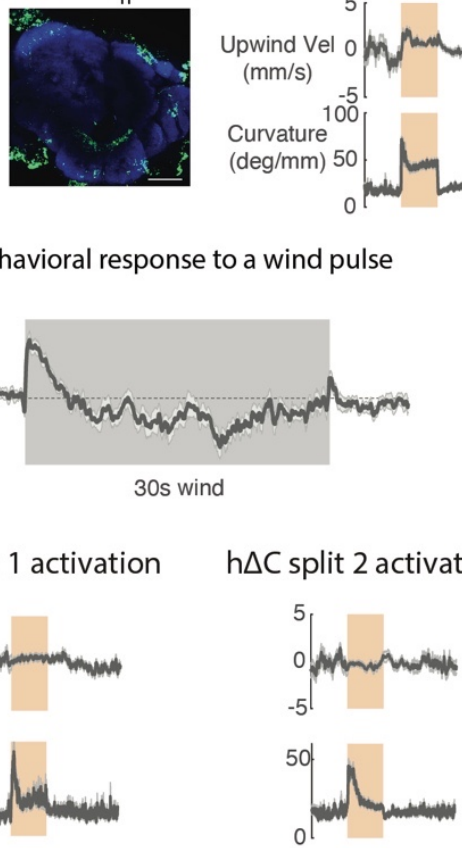


\section{Fig. S4: Additional data on FB neuron responses and behavior}

A) Schematic showing feedforward connectivity onto FB5AB from three upwind MBONs (MBON 19, MBON 12, MBON 13), one upwind LHON (AD1b2), and one downwind MBON (MBON05). Pathways converge onto FB5AB directly or indirectly through LHCENT3 and LHPV5e1. Numbers represent the average synaptic weight between each cell type and the right-sided LHCENT3 (id: 487144598), LHPV5e1 (id: 328611004), or right-sided FB5AB (id: 5813047763).

B) Number of parallel lateral horn pathways from ACV-responsive projection neurons (25) to each FB input neuron. Pathways consist of two synapses: the first between the projection neuron and lateral horn neuron, and the second between the lateral horn neuron and the FB input neuron. Blue bar represents the number of pathways converging onto FB5AB.

C) Calcium response examples from individual flies for $F B$ tangential inputs and $\mathrm{h} \triangle \mathrm{C}$. Top row depicts average $\Delta \mathrm{F} / \mathrm{F}$ for each direction across 5 trials, heat maps below depict responses in individual trials.

D) Calcium response to wind alone (5s after wind onset) vs ACV (5s after odor onset). Gray lines represent individual flies and black lines represent genotype means, except in VT062617-GAL4 where gray lines represent average increases of individual active columns. Significant increase for 21D07GAL4 and VT062617-GAL4 (paired student t-test: $p=3.9090 e-04,4.7885 e-11$ ). No significant increase for 65C03 (paired student's t-test, $p=0.7790$ ) and significant decreases for vFB split (paired student ttest; $p=0.0028$ )

E) Tangential inputs and $\mathrm{h} \Delta \mathrm{C}$ neurons are cholinergic. Left column: Flies expressing chrimson-mVenus under the control of each driver using a ChAT-LexA,LexAOP-FLP strategy (green, see Materials and Methods). Right column: Tangential inputs and $\mathrm{h} \triangle \mathrm{C}$ neurons are not GABAergic. 21D07-GAL4: same genotype as left, but co-stained with anti-GABA (magenta). There is no colocalization of GABA and Chrimson-mVenus. Flies expressing Chrimson-mVenus using a Gad1-LexA, LexAOP-FLP strategy (green) do not label FB neurons in 65C03-GAL4 or VT062617-GAL4. Scale bars represent 50 $\mu \mathrm{M}$.

F) Timecourses of upwind velocity and curvature averaged across flies (mean \pm SEM, N=27, 24, 38, 27 flies), corresponding to the data is Fig. $3 \mathrm{C}, \mathrm{D}, \mathrm{E}$ with the addition of VT062617-GAL4 (N=23).

G) Additional data for ventral FB inputs labeled by VT029515-GAL4. Left: Max-projection of confocal image of the driver. Right: Example behavioral trajectories. Scale bar represents $50 \mu \mathrm{M}$.

H) Response to a pulse of clean charcoal-filtered wind $(11.9 \mathrm{~cm} / \mathrm{s})$. Flies were genetically blind (NorpA36 in w118 (5905) background). Upwind velocity transiently increases in response to the onset of wind ( $\mathrm{N}=25$ flies).

I) Effect of silencing FB neurons on upwind velocity and offset curvature responses to 10 s $1 \% \mathrm{ACV}$. Mean \pm STD overlaid. (Mann Whitney U test comparing to UAS-TNT control: upwind: $p=0.048027$, $0.65028,0.00086718,0.60472$, offset curvature $p=0.044317,0.087747,0.31643,0.0021892)$. Crosses of 21D07 and VT062617-GAL4 to UAS-Kir were lethal and progeny could not be evaluated. Corrected for multiple comparisons using the Bonferroni method.

J) Timecourses of upwind velocity and curvature averaged across flies for $h \Delta C$ split-GAL4s, matching the data in Figure $4 \mathrm{H}(\mathrm{N}=32,27)$. 
bioRxiv preprint doi: https://doi.org/10.1101/2021.04.21.440842; this version posted April 22, 2021. The copyright holder for this preprint (which

was not certified by peer review) is the author/funder, who has granted bioRxiv a license to display the preprint in perpetuity. It is made available under aCC-BY-NC-ND 4.0 International license.

Figure 5

A

Direct Pathway

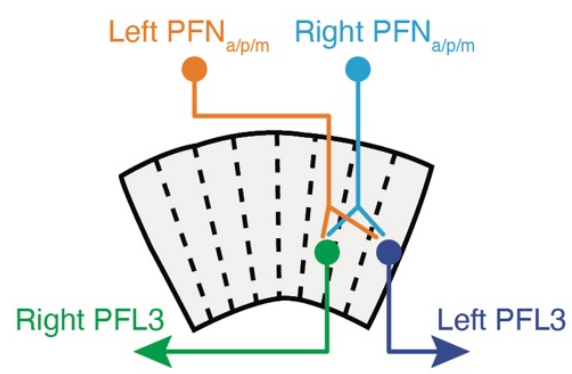

C

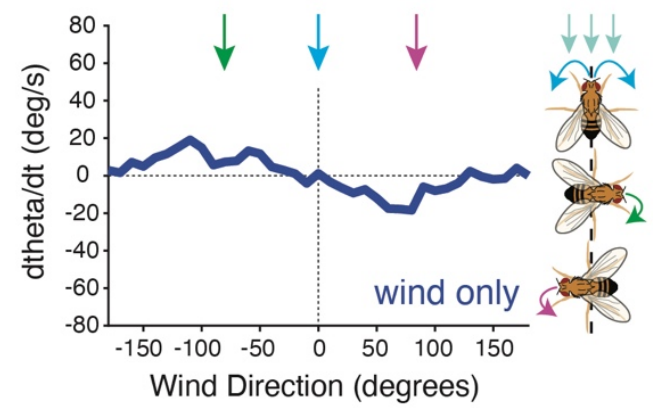

E

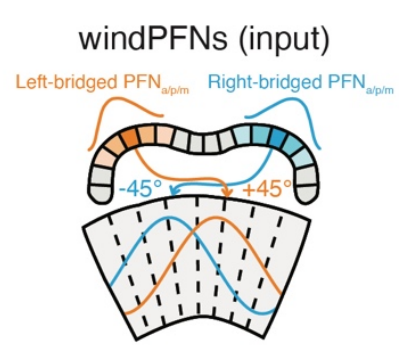

$\mathrm{H}$

$\mathrm{F}$

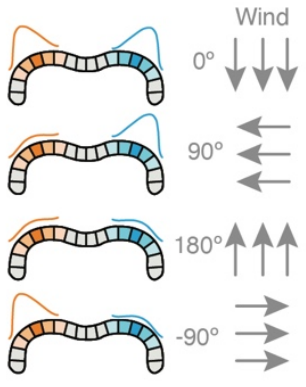

Direct Pathway
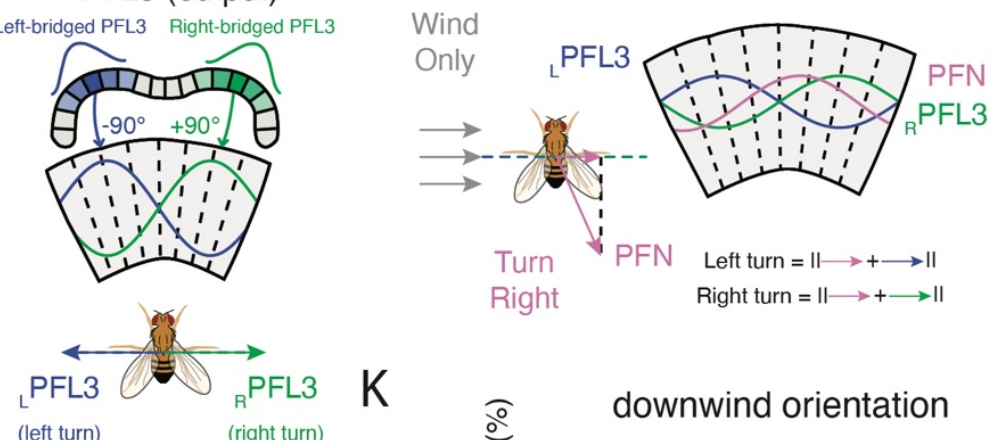

Left-bridged PFL3 (Output)

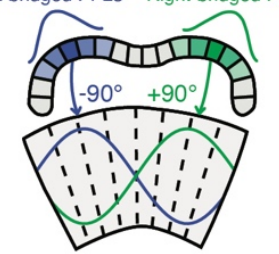

(left turn)
B

Indirect Pathway

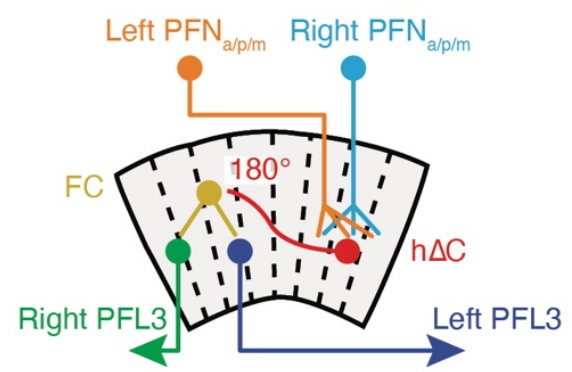

D

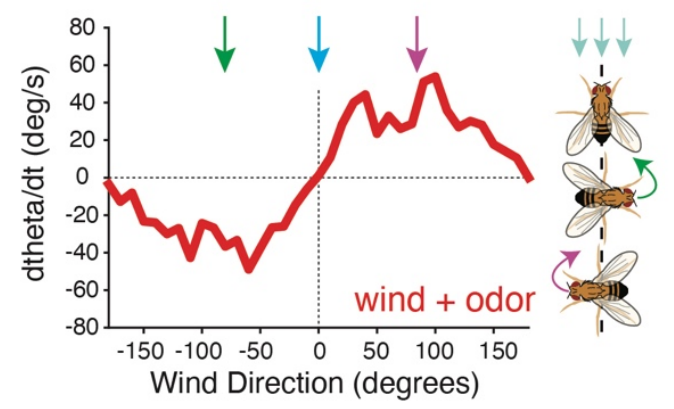

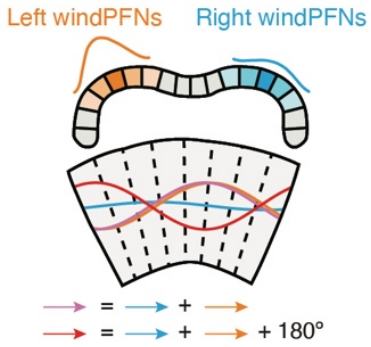

Indirect Pathway

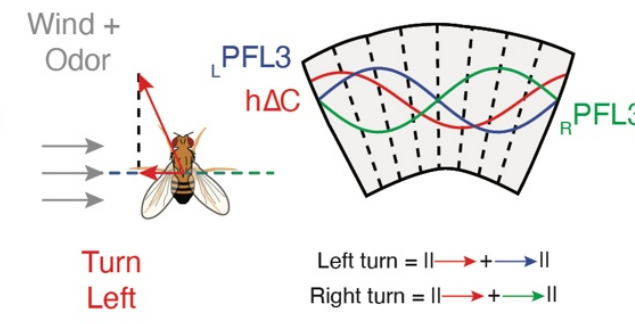

L

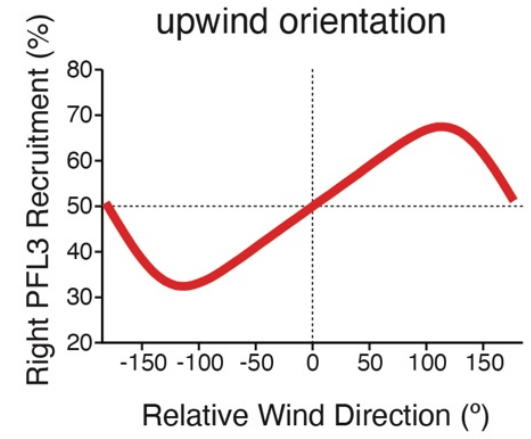


Fig. 5: A model for olfactory control of wind orientation.

A) Direct pathway from windPFNs to PFL3 pre-motor output neurons.

B) Indirect pathway from windPFNs to PFL3: left and right PFNs synapse onto h $\triangle \mathrm{C}$ neurons, which project $180^{\circ}$ across the FB to FC neurons, which finally project to PFL3 neurons.

C) Mapping from wind direction input to turning motor output derived from behavioral data. Plot shows mean angular velocity as a function of initial egocentric wind direction, computed from behavioral responses to odor shown in Fig. 1B. Mapping shown here is taken over 1 second following odor offset, in the presence of wind only. Negative wind directions (wind from left) promote positive angular velocities (right turns), leading to stable downwind orientation. Cartoons depict fly turning at $-90^{\circ}, 0^{\circ}$, and $90^{\circ}$ wind directions, as indicated by colored arrows to generate stable orientation.

D) Same as (C) for 1 second following odor onset. Here, negative wind directions (wind from left) promote negative angular velocities (left turns), leading to stable upwind orientation.

E) Predicted heading signals in windPFNs. Left and right hemisphere PFNs each receive a heading bump through the protocerebral bridge that is shifted $45^{\circ}$ contralateral from the heading signal found in compass neurons (16).

F) Predicted wind tuning in windPFNs. WindPFNs show sinusoidal tuning to wind direction that is maximal at $45^{\circ}$ ipsilateral (20). In the model, the amplitude of the activity bump on each side of the protocerebral bridge is modulated by wind direction so that its amplitude is maximal at $45^{\circ}$ ipsilateral. Panel shows activity bump amplitudes of left and right windPFNs in the protocerebral bridge (left) and vector representations (right) for four different wind directions.

G) Vector representation (left) and FB activity profiles (right) of windPFN and $\mathrm{h} \Delta \mathrm{C}$ activity. Right (blue) and left (orange) windPFNs represent vectors whose angles are $45^{\circ}$ contralateral to the fly midline, and whose amplitudes depend on wind direction. The net windPFN signal in the FB (pink) represents the sum of these two vectors. Leftward wind $\left(-90^{\circ}\right)$ generates a net PFN signal shifted towards left PFN heading bump. $\mathrm{h} \Delta \mathrm{C}$ projects half-way across the $\mathrm{FB}$, resulting in $180^{\circ}$ rotation of the net PFN vector (red). Leftward wind $\left(-90^{\circ}\right)$ generates an $\mathrm{h} \Delta \mathrm{C}$ signal closer to the right PFN heading bump.

H) Predicted heading signals in PFL3 output neurons. Left and right hemisphere PFL3 neurons each receive a heading bump through the protocerebral bridge that is shifted $90^{\circ}$ ipsilateral from the heading signal found in compass neurons (16). Panel show activity profiles (top) and vector representations (bottom).

I) Turn computation in the direct pathway. Leftward wind generates a net windPFN signal that is shifted towards the right PFL3 heading bump, leading to right turns (we assume here that PFL3 projects contralaterally but is inhibitory, leading to ipsilateral turns).

J) Turn computation in the indirect pathway. Leftward wind generates a net windPFN signal that is shifted towards the left PFL3 heading bump, leading to left turns.

K) Predicted mapping from wind direction input to turning motor output in the direct pathway. Across all wind directions, the direct pathway generates stable downwind orientations.

L) Predicted mapping from wind direction input to turning motor output in the indirect pathway. Across all wind directions, the indirect pathway generates stable upwind orientations. 
Figure S5

\section{A}
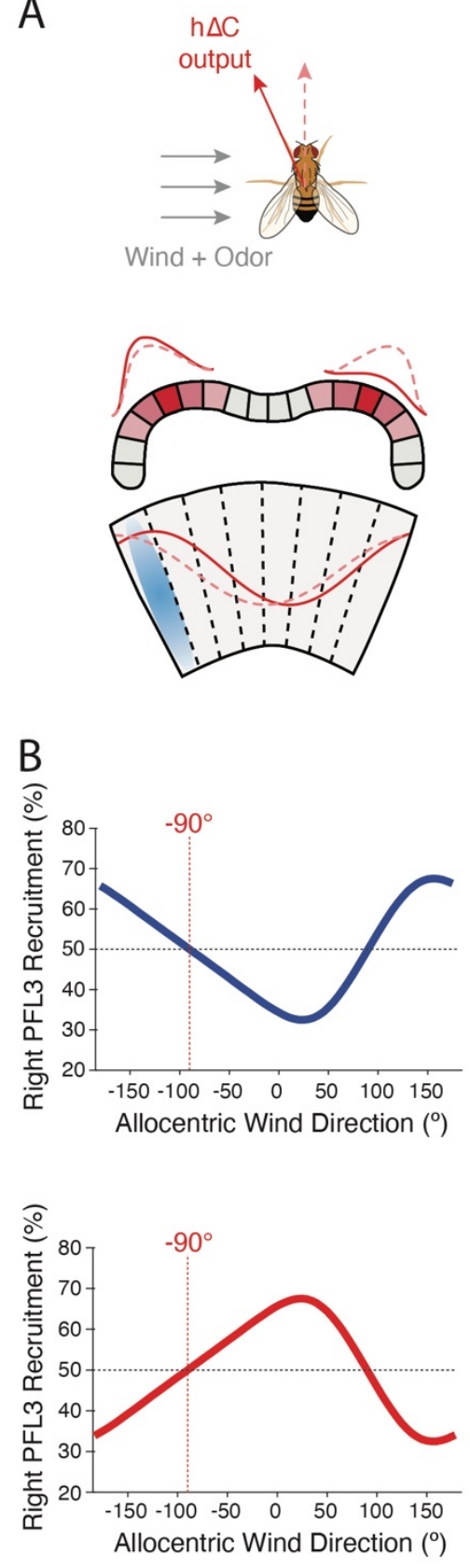
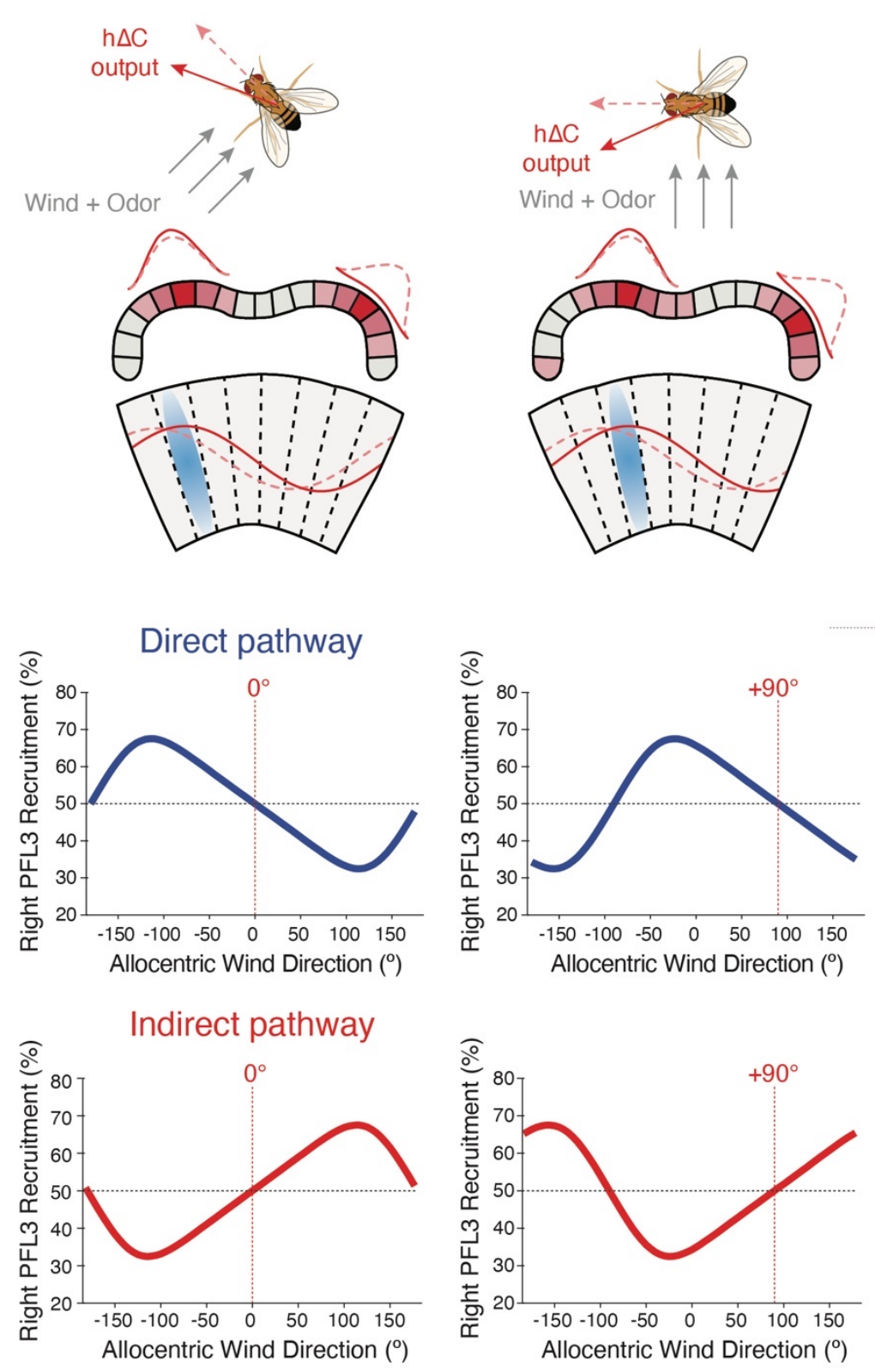

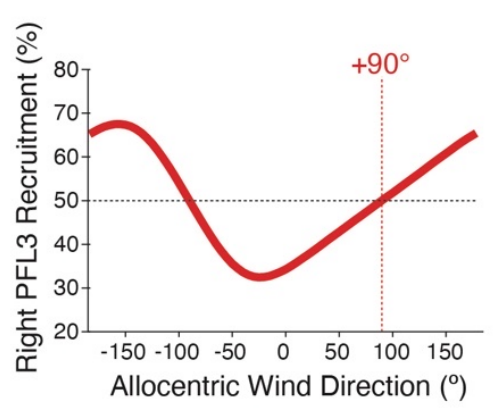

Fig. S5: Role of the heading compass in FB model of wind orientation.

A) Predicted $\mathrm{h} \triangle \mathrm{C}$ activity bump depends on both fly heading and egocentric wind direction. We consider three different scenarios with equivalent egocentric wind direction $\left(-90^{\circ}\right)$ but different heading directions (left, $0^{\circ}$; middle, $-45^{\circ}$; right, $-90^{\circ}$ ). For comparison, the $\mathrm{h} \Delta \mathrm{C}$ vector and sinusoid for frontal wind are also displayed (dashed lines). As the fly's heading rotates, the $\mathrm{h} \Delta \mathrm{C}$ activity bump shifts linearly across the FB (blue: peak of activity bump). As the allocentric wind direction changes from frontal wind to leftward wind, the $h \triangle C$ activity bump shifts by approximately one FB column, regardless of the 
bioRxiv preprint doi: https://doi.org/10.1101/2021.04.21.440842; this version posted April 22, 2021. The copyright holder for this preprint (which

was not certified by peer review) is the author/funder, who has granted bioRxiv a license to display the preprint in perpetuity. It is made available under aCC-BY-NC-ND 4.0 International license.

heading direction. Thus, heading determines the central location of the $\mathrm{h} \Delta \mathrm{C}$ activity bump, while egocentric wind direction nudges the bump to the left or right of this central location.

B) Direct and indirect pathways generate egocentric wind orientation, regardless of absolute heading direction. As the absolute heading direction (red dashed line) is rotated from $-90^{\circ}$ (left) to $0^{\circ}$ (middle) to $+90^{\circ}$ (right), the direct pathway shifts to generate stable downwind orientations at $90^{\circ}, 180^{\circ}$, and $-90^{\circ}$ wind, respectively, while the indirect pathway shifts to generate stable upwind orientations at $-90^{\circ}, 0^{\circ}$, and $90^{\circ}$ wind, respectively. 
Figure S6

A

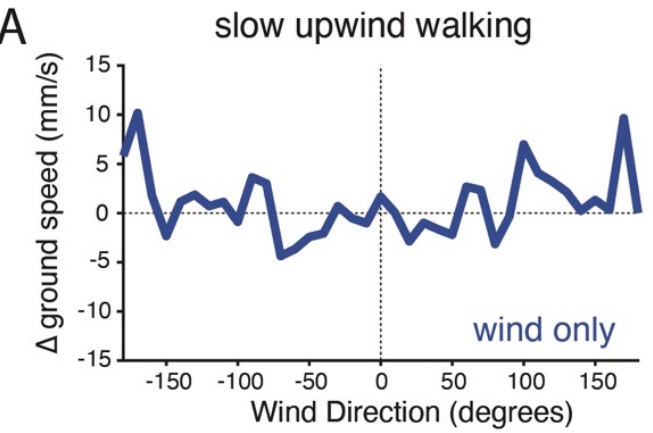

B

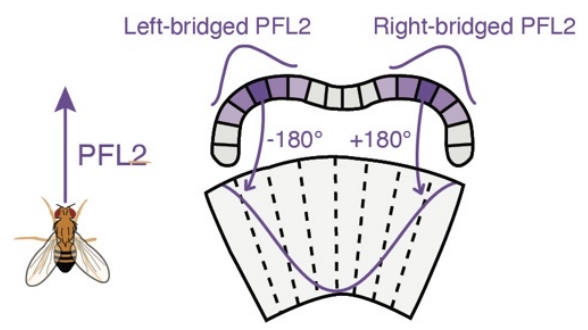

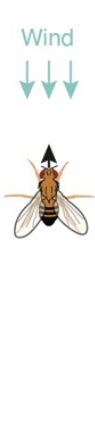

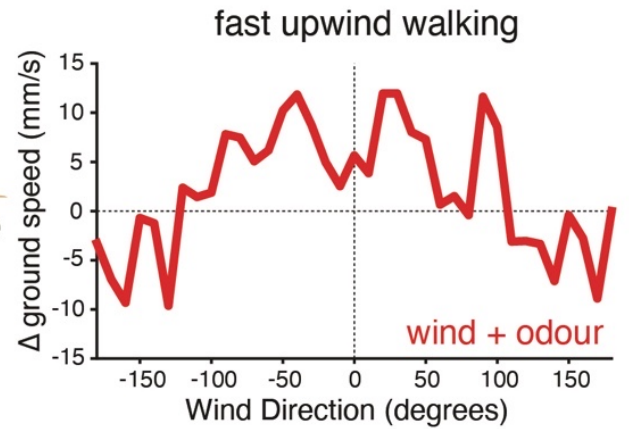

Wind
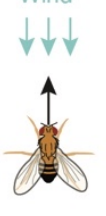

Direct Pathway

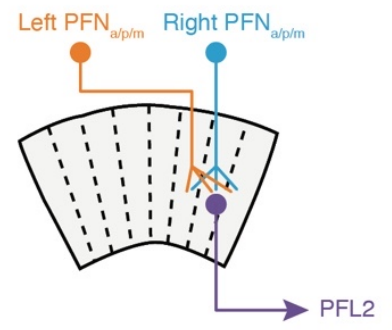

D
Indirect Pathway

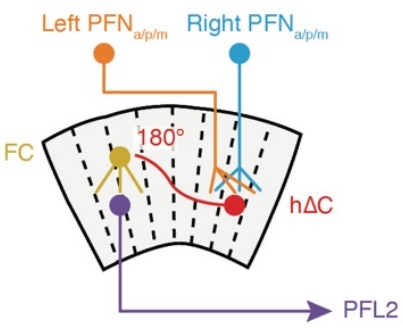

$\mathrm{E}$
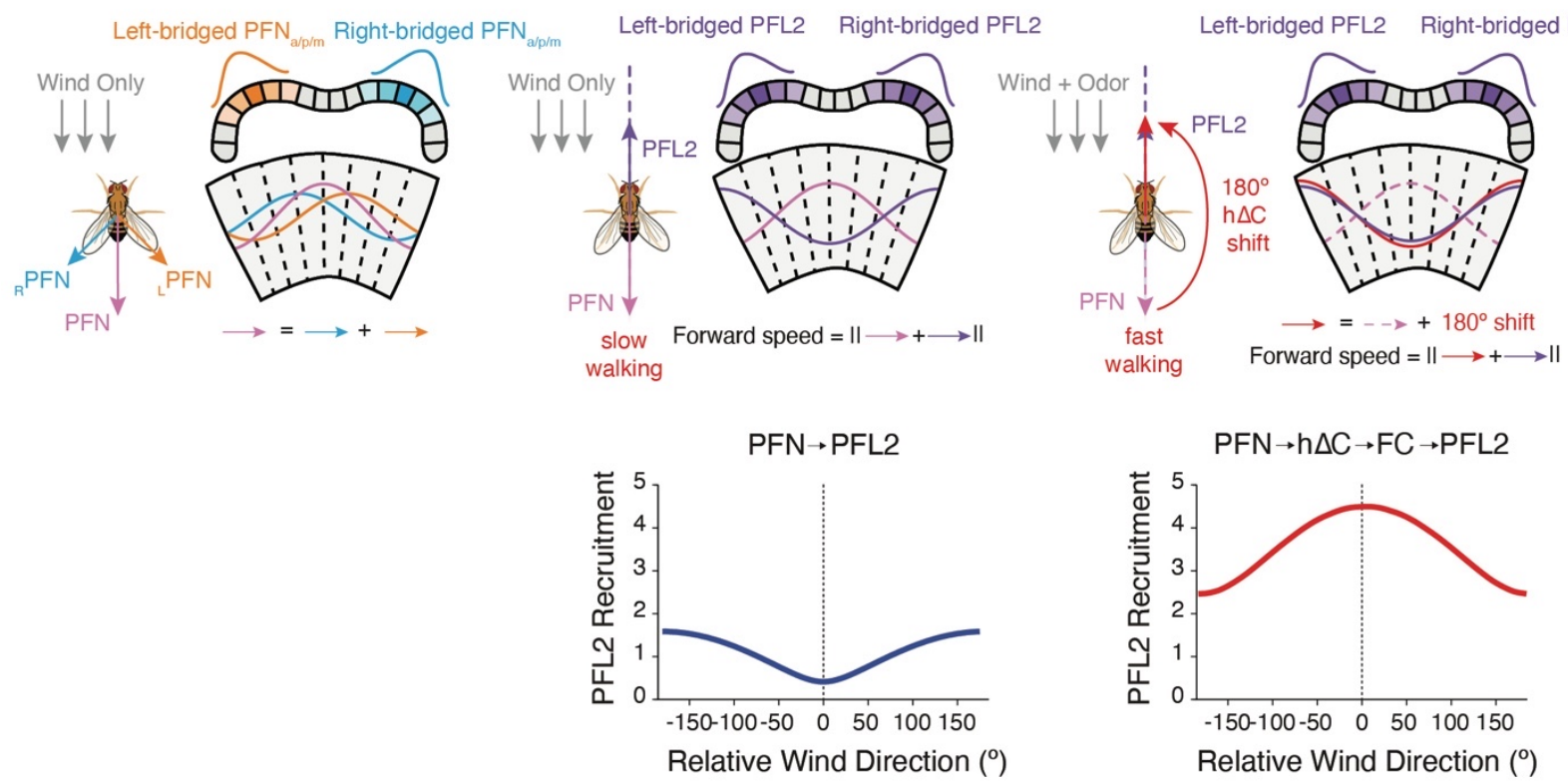

Fig. S6: A model for olfactory gating of wind-evoked forward velocity.

A) Mappings between egocentric wind direction and mean change in forward velocity, computed from odor response data shown in Fig. 1B. Positive wind directions indicate wind to the fly's right and positive ground speed indicates forward walking. Left: 0-1s after odor offset. Right: 0-1 s after odor onset. Cartoons depict fly ground speed at $0^{\circ}$ wind direction.

B) Predicted heading signals in PFL2 neurons. Left and right hemisphere PFL2 neurons each receive a heading bump through the proto-cerebral bridge that is shifted $180^{\circ}$ ipsilateral from the heading signal 
found in compass neurons (16). Right: Heading signals in PFL2 neurons are shown here both as vectors and sinusoids. Middle: In the direct pathway, left and right PFNs directly synapse with PFL2 neurons. Right: In the indirect pathway, left and right PFNs synapse onto $\mathrm{h} \Delta \mathrm{C}$ neurons, which project $180^{\circ}$ across the FB to FC neurons, which finally project to PFL2 neurons.

C) Frontal wind $\left(0^{\circ}\right)$ generates a windPFN signal centered between the left and right PFN heading bumps.

D) In the direct pathway, frontal wind generates a windPFN bump that is completely out-of-phase with the PFL2 heading bump, leading to no forward walking (top). Across all wind directions, the direct pathway generates downwind walking (bottom).

E) In the indirect pathway, frontal wind generates a windPFN bump that is shifted $180^{\circ}$ by $\mathrm{h} \Delta \mathrm{C}$, making it completely in-phase with the PFL2 heading bump and leading to forward walking (top). Across all wind directions, the direct pathway generates upwind walking (bottom). 
Figure 6

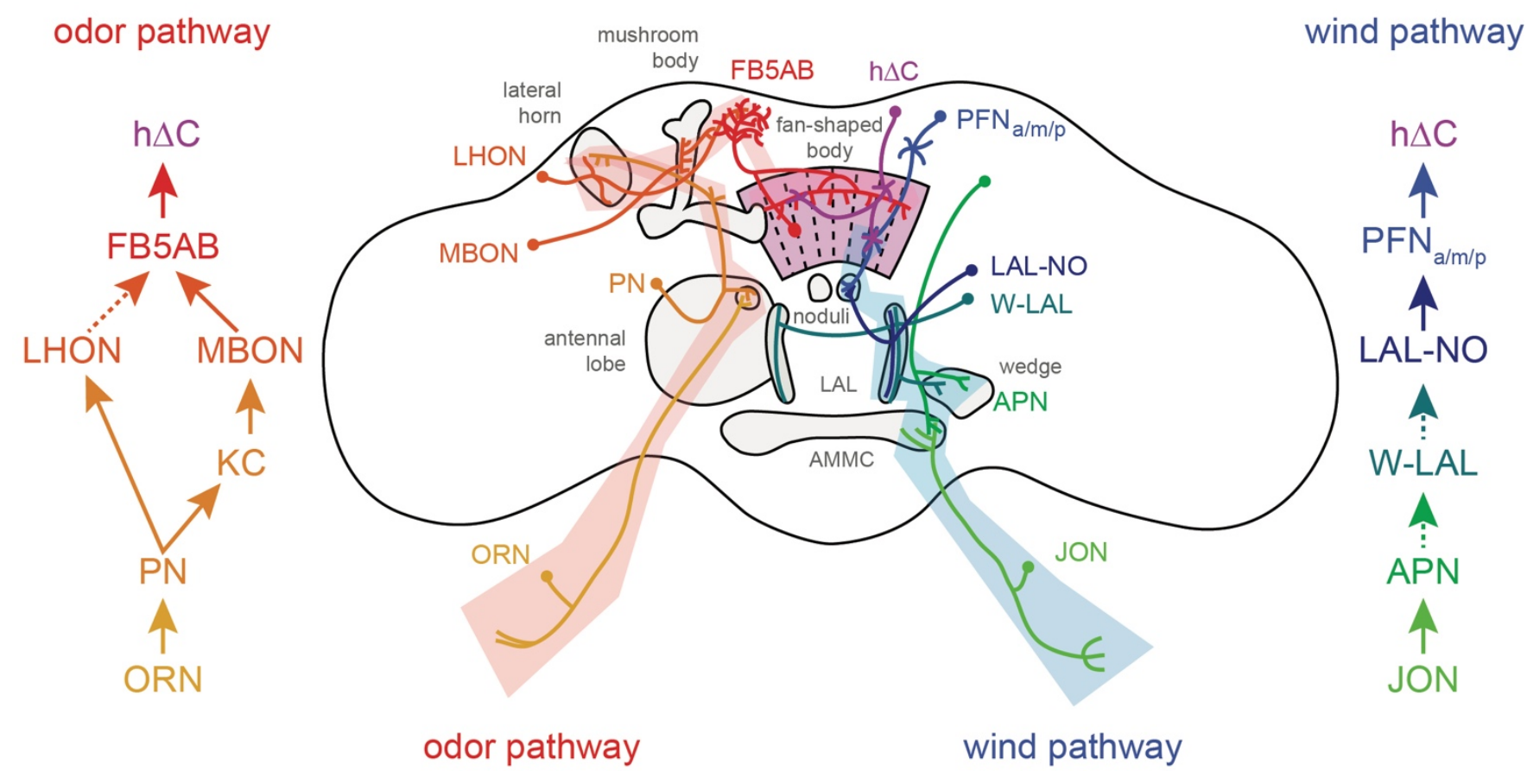

Fig. 6: Summary of odor and wind processing pathways

Schematic depicting main processing pathways for attractive odor and wind and their integration point in the FB. Neuropil regions outlined in gray. Dashed lines represent indirect connections. Odor pathway (left): odors are transduced by olfactory receptor neurons (ORNs) in the third antennal segment $(23,24)$. ORNs expressing the same olfactory receptor project to a glomerulus of the antennal lobe, where they synapse on a smaller number of projection neurons (PNs). Single antennal lobe PNs project to both the calyx of the mushroom body $(\mathrm{MB})$ and to the lateral horn $(19,27)$. LHONs (LHAD1b2) and MBONs (MBONs 05,12,13, and 19) provide output from the MB and LH $(10,26)$ and converge directly (MBON) or indirectly (LHON) onto the fan-shaped body input neuron FB5AB $(18,16)$. FB5AB makes synapses on the output tufts of $h \Delta C$ neurons. Wind pathway (right): Walking insects detect wind direction through mechanosensory Johnston's organ neurons (JONs) in the second antennal segment $(49,50)$. Wind-sensitive JONs (JONs-C and E) project to the antennal mechanosensory and motor center (AMMC, 50). AMMC projection neurons (APNs 2 and 3 ) encode displacements of one antenna (28) and project to the neighboring wedge (WED). W-LAL neurons carry displacement information to the LAL (51), while LAL-NOa neurons carry wind direction information to the noduli (20). WindPFNs (PFNa,p, and $\mathrm{m}$ ) receive wind direction information in the noduli (20) and heading information in the protocerebral bridge (16) and provide input to the dendrites of $\mathrm{h} \Delta \mathrm{C}$ neurons. 